\title{
OFF-DIAGONAL ESTIMATES FOR BI-COMMUTATORS
}

\author{
EMIL AIRTA, TUOMAS HYTÖNEN, KANGWEI LI, HENRI MARTIKAINEN, AND TUOMAS OIKARI
}

\begin{abstract}
We study the bi-commutators $\left[T_{1},\left[b, T_{2}\right]\right]$ of pointwise multiplication and Calderón-Zygmund operators, and characterize their $L^{p_{1}} L^{p_{2}} \rightarrow L^{q_{1}} L^{q_{2}}$ boundedness for several off-diagonal regimes of the mixed-norm integrability exponents $\left(p_{1}, p_{2}\right) \neq\left(q_{1}, q_{2}\right)$. The strategy is based on a bi-parameter version of the recent approximate weak factorization method.
\end{abstract}

\section{INTRODUCTION}

In this paper we characterise many new estimates for bi-commutators. The classical commutators have the form $[b, T]: f \mapsto b T f-T(b f)$, where $T$ is a singular integral operator

$$
T f(x)=\int_{\mathbb{R}^{d}} K(x, y) f(y) \mathrm{d} y .
$$

The expression (1.1) represents a broad class of linear transformations of functions arising across analysis. Distinguished special cases are the Hilbert transform $H$ in dimension $d=1$, which has the kernel $K(x, y)=\frac{1}{x-y}$, and the Riesz transforms $R_{j}$ in dimensions $d \geq 2$, which have the kernel $K_{j}(x, y)=\frac{x_{j}-y_{j}}{|x-y|^{d+1}}, j=1, \ldots, d$.

The Hilbert transform lies in the scope of complex analysis, and the Hilbert commutator $[b, H]$ is connected to Hankel operators. A classical theorem of Nehari [39] characterises the boundedness of the Hilbert commutators $[b, H]$ via this link. On the other hand, the result of Coifman-Rochberg-Weiss [6] brings the commutators to the heart of harmonic analysis by showing that

$$
\|b\|_{\mathrm{BMO}} \lesssim\|[b, T]\|_{L^{p}\left(\mathbb{R}^{d}\right) \rightarrow L^{p}\left(\mathbb{R}^{d}\right)} \lesssim\|b\|_{\mathrm{BMO}}, \quad p \in(1, \infty),
$$

for a class of non-degenerate singular integrals $T$ on $\mathbb{R}^{d}$. Here BMO stands for the usual space of functions of bounded mean oscillation:

$$
\|b\|_{\mathrm{BMO}}:=\sup _{I} f_{I}\left|b-\langle b\rangle_{I}\right|
$$

where the supremum is over all cubes $I \subset \mathbb{R}^{d}$ and $\langle b\rangle_{I}=f_{I} b:=\frac{1}{|T|} \int_{I} b$.

The off-diagonal situation $[b, T]: L^{p} \rightarrow L^{q}, p \neq q$, is also completely understood. In the case $1<p<q<\infty$, a two-sided estimate like (1.2) with BMO replaced by the

2010 Mathematics Subject Classification. 42B20.

Key words and phrases. Calderón-Zygmund operators, singular integrals, multi-parameter analysis, commutators. 
homogeneous Hölder space $\dot{C}^{0, \alpha}$,

$$
\|b\|_{\dot{C}^{0, \alpha}}:=\sup _{x \neq y} \frac{|b(x)-b(y)|}{|x-y|^{\alpha}}, \quad \alpha:=d\left(\frac{1}{p}-\frac{1}{q}\right),
$$

was obtained by Janson [28]. The remaining range with $1<q<p<\infty$ was characterised only very recently in [22]:

$$
\|[b, T]\|_{L^{p} \rightarrow L^{q}} \sim\|b\|_{\dot{L}^{r}}:=\inf _{c \in \mathbb{C}}\|b-c\|_{L^{r}}, \quad \frac{1}{r}:=\frac{1}{q}-\frac{1}{p} .
$$

As the commutator annihilates constants, all of the spaces above also have the feature that they do not see constants. This philosophy is more complicated in the bi-parameter setting where the bi-commutator annihilates all functions that depend only on one of the variables $\left(x_{1}, x_{2}\right) \in \mathbb{R}^{d_{1}} \times \mathbb{R}^{d_{2}}$.

The motivation for commutator estimates stems from their many applications and connections to modern harmonic analysis. For example, the $L^{p} \rightarrow L^{p}$ characterization yields factorizations for Hardy spaces [6] and implies various div-curl lemmas relevant for compensated compactness [7]. In [22], the off-diagonal $L^{p} \rightarrow L^{q}$ boundedness for $q<p$ is connected to a conjecture of Iwaniec [27] about the prescribed Jacobian problem.

In this paper, we address the question of the off-diagonal $L^{p}\left(\mathbb{R}^{d}\right) \rightarrow L^{q}\left(\mathbb{R}^{d}\right)$ boundedness of the bi-commutators $\left[T_{1},\left[b, T_{2}\right]\right]$, where each $T_{i}$ is a Calderón-Zygmund operator on $\mathbb{R}^{d_{i}}$ and $\mathbb{R}^{d}$ is viewed as the bi-parameter product space $\mathbb{R}^{d}=\mathbb{R}^{d_{1}} \times \mathbb{R}^{d_{2}}$. Due to the product space nature of the problem, it is natural to introduce an additional level of generality by allowing different integrability exponents in the $x_{1}$ and $x_{2}$ coordinates, thereby leading to the question of $L_{x_{1}}^{p_{1}} L_{x_{2}}^{p_{2}} \rightarrow L_{x_{1}}^{q_{1}} L_{x_{2}}^{q_{2}}$ boundedness. In contrast to the three qualitatively different regimes $p<q, p=q$ and $p>q$ for the commutator, there will now be nine different situations depending on the relative size of both $p_{1}, q_{1}$ on the one hand, and $p_{2}, q_{2}$ on the other hand.

We are interested specifically on the off-diagonal case $\left(p_{1}, p_{2}\right) \neq\left(q_{1}, q_{2}\right)$. The diagonal, involving the product BMO space of Chang and Fefferman [4, 5], has e.g. been studied in Ferguson-Sadosky [13] and Ferguson-Lacey [14], with further extensions appearing in [29, 30, 42].

Our question is the natural bi-parameter analogue of [22]. In fact, [22] already suggests a naive conjecture, where in each regime of the exponents, we should have the corresponding natural vector-valued space. For example, in the case

$$
p_{1}<q_{1} \text { and } p_{2}=q_{2}
$$

we could expect the space $\dot{C}_{x_{1}}^{0, \beta_{1}}\left(\mathrm{BMO}_{x_{2}}\right)$ and in the case

$$
p_{1}=q_{1} \text { and } p_{2}<q_{2}
$$

we could expect the space $\mathrm{BMO}_{x_{1}}\left(\dot{C}_{x_{2}}^{0, \beta_{2}}\right)$, where $\beta_{i}:=d_{i}\left(\frac{1}{p_{i}}-\frac{1}{q_{i}}\right)$. Somewhat strikingly, we show that the naive conjecture is not always the right one. In the second case, the space $\dot{C}_{x_{2}}^{0, \beta_{2}}\left(\mathrm{BMO}_{x_{1}}\right)$, where the order of the spaces $\dot{C}_{x_{2}}^{0, \beta_{2}}$ and $\mathrm{BMO}_{x_{1}}$ has been switched to an unexpected order, provides the correct sufficient and necessary condition.

The following Theorem 1.3 is our main result. It verifies the naive conjecture in some cases and proves an unexpected necessary and sufficient condition in some other cases. Some characterisations are left for future work as they do not appear to be amenable to our current methods. The stated theorem is a simplified and shortened version of the 
obtained new estimates - for example, the symmetry assumption is used here purely for convenience in order not to have to include partial adjoints $\left[T_{1}^{*},\left[b, T_{2}\right]\right]$ in the estimates. In the three lower-right cases of the table of Theorem 1.3, where only an upper bound is stated, we also obtain certain related lower bounds, but these do not admit a simple formulation in terms of classical function spaces, and are therefore omitted in this Introduction.

1.3. Theorem. Let $T_{1}$ and $T_{2}$ be two symmetrically non-degenerate CZOs on $\mathbb{R}^{d_{1}}$ and $\mathbb{R}^{d_{2}}$, respectively, $b \in L_{\text {loc }}^{2}\left(\mathbb{R}^{d_{1}+d_{2}}\right)$ and $p_{1}, p_{2}, q_{1}, q_{2} \in(1, \infty)$. Let

$$
\beta_{i}:=d_{i}\left(\frac{1}{p_{i}}-\frac{1}{q_{i}}\right), \quad \text { if } \quad p_{i}<q_{i} ; \quad \frac{1}{r_{i}}:=\frac{1}{q_{i}}-\frac{1}{p_{i}}, \quad \text { if } \quad p_{i}>q_{i} .
$$

Then $\left\|\left[T_{1},\left[b, T_{2}\right]\right]\right\|_{L_{x_{1}}^{p_{1}} L_{x_{2}}^{p_{2}} \rightarrow L_{x_{1}}^{q_{1}} L_{x_{2}}^{q_{2}}}$ has upper and lower bounds according to the following table:

\begin{tabular}{|c|c|c|c|}
\hline & $p_{1}<q_{1}$ & $p_{1}=q_{1}$ & $p_{1}>q_{1}$ \\
\hline$p_{2}<q_{2}$ & $\sim\|b\|_{\dot{C}_{x_{1}}^{0, \beta_{1}}\left(\dot{C}_{x_{2}}^{0, \beta_{2}}\right)}$ & $\sim\|b\|_{\dot{C}_{x_{2}}^{0, \beta_{2}}\left(\mathrm{BMO}_{x_{1}}\right)}$ & $\begin{array}{l}\lesssim\|b\|_{\dot{L}_{x_{1}}^{r_{1}}\left(\dot{C}_{x_{2}}^{0, \beta_{2}}\right)} \\
\gtrsim\|b\|_{\dot{C}_{x_{2}}^{0, \beta_{2}}\left(\dot{L}_{x_{1}}^{r_{1}}\right)}\end{array}$ \\
\hline$p_{2}=q_{2}$ & $\sim\|b\|_{\dot{C}_{x_{1}}^{0, \beta_{1}}\left(\mathrm{BMO}_{x_{2}}\right)}$ & $\begin{array}{l}\lesssim\|b\|_{\mathrm{BMO}_{\mathrm{prod}}} \\
\gtrsim\|b\|_{\mathrm{BMO}_{\mathrm{rect}}}\end{array}$ & $\lesssim\|b\|_{\dot{L}_{x_{1}}^{r_{1}}\left(\mathrm{BMO}_{x_{2}}\right)}$ \\
\hline$p_{2}>q_{2}$ & $\sim\|b\|_{\dot{C}_{x_{1}}^{0, \beta_{1}}\left(\dot{L}_{x_{2}}^{r_{2}}\right)}$ & $\lesssim\|b\|_{\mathrm{BMO}_{x_{1}}\left(\dot{L}_{x_{2}}^{r_{2}}\right)}$ & $\lesssim\|b\|_{\dot{L}_{x_{1}}^{r_{1}}\left(\dot{L}_{x_{2}}^{r_{2}}\right)}$ \\
\hline
\end{tabular}

It is important to realize that commutator upper bounds are expected for all bounded singular integrals, while the lower bounds obviously require some non-degeneracy. All our lower bounds hold under weak non-degeneracy assumptions on the operators, and neither the homogeneity nor the translation-invariance of the operators is needed. However, they have been crucial in the diagonal case [14, 29].

There has been a vast amount of other recent activity regarding both one-parameter and multi-parameter commutators. For example, the two-weight commutator estimates, which include [17, 18, 19, 22, 31, 32, 33, 34], have been one of the main lines of development. Commutators are also actively studied in other settings: see for example [10] for the flag setting, [11] for the Zygmund dilation setting and [12] for the Bessel setting.

The motivation for this paper is not just the results in Theorem 1.3 but also the associated methodology. We develop some interesting bi-parameter versions of the flexible one-parameter commutator methods [22]. In more detail, our strategy requires us to develop new bi-parameter methods that exploit the following known interplay between commutator bounds and weak factorizations. The logic of this connection is that while Nehari's theorem [39] may be seen as a corollary of the classical factorization of $H^{1}(\mathbb{D})$, Coifman, Rochberg and Weiss [6] reversed the reasoning and deduced from the commutator estimate (1.2), using duality, a weak factorization (involving sums of products, rather than just products) of the real-variable Hardy space $H^{1}\left(\mathbb{R}^{d}\right)$. This connection between commutators estimates and factorizations of functions stems from identities like

$$
\langle[b, T] f, g\rangle=\left\langle b, T f \cdot g-f \cdot T^{*} g\right\rangle .
$$

Recently, in [22] the idea was to reverse the reasoning again, and to first directly prove a suitable factorization, and then use it to prove the desired commutator estimate. This 
leads to the approximate weak factorization method, where a function $h$ is expanded in the form

$$
h=\frac{h}{T^{*} g} T^{*} g=:-f \cdot T^{*} g=-f \cdot T^{*} g+T f \cdot g+h^{\prime}, \quad h^{\prime}:=-T f \cdot g,
$$

and a suitable $g$ above allows to absorb the error $\left\|h^{\prime}\right\| \ll\|h\|$.

Instead of (1.4), in the bi-parameter setting we have

$$
\left\langle\left[T_{1},\left[b, T_{2}\right]\right] f, g\right\rangle=\left\langle b, T_{2} f \cdot T_{1}^{*} g-f \cdot T_{1}^{*} T_{2}^{*} g-T_{1} T_{2} f \cdot g+T_{1} f \cdot T_{2}^{*} g\right\rangle .
$$

We develop our new weak factorizations in Section 4. In Lemma 4.4 we expand a function $f$ supported on a rectangle $R=I \times J$ and satisfying $\int_{I} f=\int_{J} f=0$ using the approximate weak factorization logic. This produces more error terms, with more complicated supports, when compared to the one-parameter analogue (1.5), and makes the repeated use of the factorization more tricky. Nevertheless, we manage to use our biparameter approximate weak factorization to e.g. prove Theorem 4.18, which is the key to many commutator lower bounds.

Here is an outline of the paper. After the short preliminaries of Section 2 we move on to the oscillatory characterizations of various function spaces in Section 3 . These function space characterizations will be combined with the weak factorizations of Section 4 to prove our necessary conditions for commutator boundedness in Section 5 . Section 6 collects all the sufficient conditions. Sections 5 and 6 combined give Theorem 1.3 . Section 7 records some additional vector-valued estimates for commutators that are of independent interest - see e.g. Theorem 7.11

Acknowledgements. E. Airta, T. Hytönen, H. Martikainen and T. Oikari were supported by the Academy of Finland through project Nos. 294840 (Martikainen), 327271 (Airta, Martikainen), 306901 (Airta, Martikainen, Oikari), and 314829 (Hytönen), and through the Finnish Centre of Excellence in Analysis and Dynamics Research (project No. 307333, all four). Martikainen and Oikari were also supported by the three-year research grant 75160010 of the University of Helsinki.

\section{PRELIMinaries}

2.A. Basic notation. We denote $A \lesssim B$ if $A \leq C B$ for some absolute constant $C$. The constant $C$ can at least depend on the dimensions of the appearing Euclidean spaces, on integration exponents, and on various Banach space constants. It can also depend on various other fixed constants, like those related to singular integrals, and so on. We denote $A \sim B$ if $B \lesssim A \lesssim B$.

When we consider $\mathbb{R}^{d}$ as a bi-parameter product space $\mathbb{R}^{d}=\mathbb{R}^{d_{1}} \times \mathbb{R}^{d_{2}}$ we often denote the mixed-norm space $L^{p_{1}}\left(\mathbb{R}^{d_{1}} ; L^{p_{2}}\left(\mathbb{R}^{d_{2}}\right)\right)$ by $L_{x_{1}}^{p_{1}} L_{x_{2}}^{p_{2}}$ - this is suggested by the notation $x=\left(x_{1}, x_{2}\right) \in \mathbb{R}^{d}=\mathbb{R}^{d_{1}} \times \mathbb{R}^{d_{2}}$. We also always identify $f: \mathbb{R}^{d} \rightarrow \mathbb{C}$ satisfying

$$
\left(\int_{\mathbb{R}^{d_{1}}}\left(\int_{\mathbb{R}_{d_{2}}}\left|f\left(x_{1}, x_{2}\right)\right|^{p_{2}} \mathrm{~d} x_{2}\right)^{p_{1} / p_{2}} \mathrm{~d} x_{1}\right)^{1 / p_{1}}<\infty
$$

with the function $\phi_{f} \in L^{p_{1}}\left(\mathbb{R}^{d_{1}} ; L^{p_{2}}\left(\mathbb{R}^{d_{2}}\right)\right), \phi_{f}\left(x_{1}\right)=f\left(x_{1}, \cdot\right)$.

Often integral pairings - denoted with the bracked notation $\langle f, g\rangle=\int f g$ - need to be taken with respect to one of the variables only. For example, if $f: \mathbb{R}^{d} \rightarrow \mathbb{C}$ and $h: \mathbb{R}^{d_{1}} \rightarrow$ 
$\mathbb{C}$, then $\langle f, h\rangle_{1}: \mathbb{R}^{d_{2}} \rightarrow \mathbb{C}$ is defined by

$$
\langle f, h\rangle_{1}\left(x_{2}\right)=\int_{\mathbb{R}^{d_{1}}} f\left(y_{1}, x_{2}\right) h\left(y_{1}\right) \mathrm{d} y_{1} .
$$

We denote averages by

$$
\langle f\rangle_{A}=f_{A} f:=\frac{1}{|A|} \int_{A} f,
$$

where $|A|$ is the Lebesgue measure of the set $A$. The indicatator function of a set $A$ is denoted by $1_{A}$. We try to denote cubes in $\mathbb{R}^{d_{i}}$ by $I_{i}, J_{i}, L_{i}$ and so on - that is, the dimension of the cube can be read from the subscript. Various rectangles then take the form $I_{1} \times I_{2}, J_{1} \times J_{2}$, etc. The side length of a cube $I_{i}$ is denoted by $\ell\left(I_{i}\right)$.

2.B. Singular integrals and commutators. We call

$$
K_{i}: \mathbb{R}^{d_{i}} \times \mathbb{R}^{d_{i}} \backslash\left\{\left(x_{i}, y_{i}\right) \in \mathbb{R}^{d_{i}} \times \mathbb{R}^{d_{i}}: x_{i}=y_{i}\right\} \rightarrow \mathbb{C}
$$

a standard Calderón-Zygmund kernel on $\mathbb{R}^{d_{i}}$ if we have

$$
\left|K\left(x_{i}, y_{i}\right)\right| \leq \frac{C}{\left|x_{i}-y_{i}\right|^{d_{i}}}
$$

and, for some $\alpha_{i} \in(0,1]$, we have

$$
\left|K\left(x_{i}, y_{i}\right)-K\left(x_{i}^{\prime}, y_{i}\right)\right|+\left|K\left(y_{i}, x_{i}\right)-K\left(y_{i}, x_{i}^{\prime}\right)\right| \leq C \frac{\left|x_{i}-x_{i}^{\prime}\right|^{\alpha}}{\left|x_{i}-y_{i}\right|^{d_{i}+\alpha_{i}}}
$$

whenever $\left|x_{i}-x_{i}^{\prime}\right| \leq\left|x_{i}-y_{i}\right| / 2$.

Many of our results hold with (2.1) replaced by a significantly weaker assumption see Remark 4.6. However, we do not emphasise this too much as it is not a novelty of this paper (see e.g. [22]).

A singular integral operator (SIO) is a linear operator $T_{i}$ on $\mathbb{R}^{d_{i}}$ (initially defined, for example, on bounded and compactly supported functions) so that there is a standard kernel $K_{i}$ for which

$$
\left\langle T_{i} f, g\right\rangle=\iint_{\mathbb{R}^{d_{i} \times \mathbb{R}^{d_{i}}}} K_{i}\left(x_{i}, y_{i}\right) f\left(y_{i}\right) g\left(x_{i}\right) \mathrm{d} y_{i} \mathrm{~d} x_{i}
$$

whenever the functions $f$ and $g$ are nice and have disjoint supports. A Calderón-Zygmund operator $(\mathrm{CZO})$ is an SIO $T_{i}$, which is bounded from $L^{p}\left(\mathbb{R}^{d_{i}}\right) \rightarrow L^{p}\left(\mathbb{R}^{d_{i}}\right)$ for all (equivalently for some) $p \in(1, \infty)$. The $T 1$ theorem [9] says that an SIO is a CZO if and only if

$$
\int_{I_{i}}\left|T_{i} 1_{I_{i}}\right|+\int_{I_{i}}\left|T_{i}^{*} 1_{I_{i}}\right| \lesssim\left|I_{i}\right|
$$

for all cubes $I_{i} \subset \mathbb{R}^{d_{i}}$. Here $T_{i}^{*}$ is the linear adjoint of $T_{i}$. We know a lot about the structure of a CZO $T_{i}$ : we can represent $T_{i}$ with certain dyadic model operators (DMOs) - see [20, 21]. We will have use for this later.

If $b \in L_{\text {loc }}^{s}\left(\mathbb{R}^{d}\right)$ for some $s \in(1, \infty)$ and $T$ is a CZO on $\mathbb{R}^{d}$, then the pairing

$$
\langle[b, T] f, g\rangle=\langle T f, b g\rangle-\langle T(b f), g\rangle
$$


is well-defined for $f, g \in L_{c}^{\infty}$. If $\mathbb{R}^{d}=\mathbb{R}^{d_{1}} \times \mathbb{R}^{d_{2}}$ and $T_{i}$ is a CZO on $\mathbb{R}^{d_{i}}$, we can similarly define $\left\langle\left[T_{1},\left[b, T_{2}\right]\right] f, g\right\rangle$. Then we can ask, via duality, if $\left[T_{1},\left[b, T_{2}\right]\right]$ maps $L_{x_{1}}^{p_{1}} L_{x_{2}}^{p_{2}} \rightarrow$ $L_{x_{1}}^{q_{1}} L_{x_{2}}^{q_{2}}$. However, when a commutator lower bound is proved, the full norm

$$
\left\|\left[T_{1},\left[b, T_{2}\right]\right]\right\|_{L_{x_{1}}^{p_{1}} L_{x_{2}}^{p_{2}} \rightarrow L_{x_{1}}^{q_{1}} L_{x_{2}}^{q_{2}}}
$$

is not actually needed. We will define so-called off-support versions of the norm, which can be defined even if we only have $b \in L_{\mathrm{loc}}^{1}$. In fact, these off-support constants depend only on the kernels $K_{1}$ and $K_{2}$ and not on the CZOs themselves. tion.

When we are given a CZO $T_{i}$ we always write $K_{i}$ for its kernel without explicit men-

\section{FUNCTION SPACES AND OSCILLATORY CHARACTERIZATIONS}

In our goal of linking the boundedness of the commutator $\left[T_{1},\left[b, T_{2}\right]\right]$ with the membership of $b$ in a suitable function space, a useful intermediate notion is provided by various oscillatory characterizations of the different function space norms. In this section we specifically go through all the oscillatory conditions that appear in our commutator lower bounds.

3.A. One-parameter spaces. We begin by recalling the relevant characterizations in the one-parameter situation, as this will motivate the necessarily more complicated expressions in the two parameter case.

The case of $\mathrm{BMO}$ is most immediate, as the norm is directly given by the oscillatory quantity

$$
\|b\|_{\mathrm{BMO}\left(\mathbb{R}^{d}\right)}:=\sup _{I} f_{I}\left|b-\langle b\rangle_{I}\right| .
$$

For the homogeneous Hölder norms

$$
\|b\|_{\dot{C}^{0, \alpha}\left(\mathbb{R}^{d}\right)}:=\sup _{x \neq y} \frac{|b(x)-b(y)|}{|x-y|^{\alpha}}
$$

there is a well-known analogous equivalent norm:

3.1. Proposition. We have

$$
\|b\|_{\dot{C}^{0, \alpha}\left(\mathbb{R}^{d}\right)} \sim \sup _{I} \frac{1}{\ell(I)^{\alpha}} f_{I}\left|b-\langle b\rangle_{I}\right|,
$$

where the supremum is over all cubes $I \subset \mathbb{R}^{d}$.

Proof. " $\gtrsim$ " is immediate, since $\left|b(x)-\langle b\rangle_{I}\right| \leq f_{I}|b(x)-b(y)| \mathrm{d} y$, where $|b(x)-b(y)| \lesssim$ $\|b\|_{\dot{C}^{0, \alpha}\left(\mathbb{R}^{d}\right)} \ell(I)^{\alpha}$ for all $x, y \in I$.

For " $\lesssim$ ", denote the right-hand side of the claim by $N$, and fix $x \neq y \in \mathbb{R}^{d}$. Define $x_{k}:=\left(1-2^{-k}\right) x+2^{-k} y$ and $y_{k}:=\left(1-2^{-k}\right) y+2^{-k} x$, and note that $x_{1}=y_{1}=\frac{1}{2}(x+y)$. If $Q_{k}(x):=Q\left(x_{k}, 2^{-k}|x-y|\right)$ is the cube of centre $x_{k}$ and side-length $2 \cdot 2^{-k}|x-y|$, we easily check that $Q_{k+1}(x) \subset Q_{k}(x)$ and $Q_{k}(x) \subset B\left(x, C 2^{-k}|x-y|\right)$. Thus, we have

$$
b(x)=\sum_{k=1}^{\infty}\left(f_{Q_{k+1}(x)} b-f_{Q_{k}(x)} b\right)+f_{Q\left(\frac{1}{2}(x+y), \frac{1}{2}|x-y|\right)} b
$$


and hence

$$
\begin{aligned}
\left|b(x)-f_{Q\left(\frac{1}{2}(x+y), \frac{1}{2}|x-y|\right)} b\right| & \leq \sum_{k=1}^{\infty} f_{Q_{k+1}(x)}\left|b-\langle b\rangle_{Q_{k}(x)}\right| \lesssim \sum_{k=1}^{\infty} f_{Q_{k}(x)}\left|b-\langle b\rangle_{Q_{k}(x)}\right| \\
& \lesssim N \sum_{k=1}^{\infty} \ell\left(Q_{k}(x)\right)^{\alpha} \lesssim N|x-y|^{\alpha} \sum_{k=1}^{\infty} 2^{-k \alpha} \lesssim N|x-y|^{\alpha} .
\end{aligned}
$$

A similar bound for $y$ in place of $x$ and the triangle inequality show that $|b(x)-b(y)| \lesssim$ $N|x-y|^{\alpha}$.

The final space of interest in the one-parameter case is

$$
\|b\|_{\dot{L}^{r}\left(\mathbb{R}^{d}\right)}:=\inf _{c}\|b-c\|_{L^{r}}
$$

where the infimum is taken over all constants. For this space we have the following characterisation - similar estimates already appeared in [22], but we single them out here as a separate proposition. A collection of cubes $\mathscr{S}$ is called $\gamma$-sparse if there are pairwise disjoint subsets $E(S) \subset S, S \in \mathscr{S}$, with $|E(S)| \geq \gamma|S|$. We can often work with $\gamma=1 / 2$ and simply talk about sparseness.

3.2. Proposition. For $r \in(1, \infty)$ we have

$$
\begin{aligned}
\|b\|_{L^{r}\left(\mathbb{R}^{d}\right)} & \sim \sup _{Q}\left\|b-\langle b\rangle_{Q}\right\|_{L^{r}(Q)} \sim\left\|M^{\#} b\right\|_{L^{r}\left(\mathbb{R}^{d}\right)} \\
& \sim \sup \left\{\sum_{S \in \mathscr{S}} \lambda_{S} \int_{S}\left|b-\langle b\rangle_{S}\right|: \mathscr{S} \text { is sparse, } \sum_{S \in \mathscr{S}}|S| \lambda_{S}^{r^{\prime}} \leq 1\right\},
\end{aligned}
$$

where $M^{\#} b$ is the sharp maximal function $M^{\#} b(x)=\sup _{Q \ni x} f_{Q}\left|b-\langle b\rangle_{Q}\right|$ and the supremum is taken over all cubes $Q \subset \mathbb{R}^{d}$.

Proof. We prove a chain of upper bounds both starting and finishing with $\|b\|_{\dot{L}^{r}\left(\mathbb{R}^{d}\right)}$, and covering all other expressions as intermediate steps. To begin with, we have

$$
\|b\|_{\dot{L}^{r}\left(\mathbb{R}^{d}\right)} \lesssim \sup _{Q}\left\|b-\langle b\rangle_{Q}\right\|_{L^{r}(Q)}=: N .
$$

To see this, fix an increasing sequence of cubes $\left(Q_{i}\right)_{i}$ that exhaust $\mathbb{R}^{d}$. Let $i \leq j$ and write

$$
\begin{aligned}
\left|\langle b\rangle_{Q_{j}}-\langle b\rangle_{Q_{i}}\right| & =\left(f_{Q_{i}}\left|\left[\langle b\rangle_{Q_{j}}-b(x)\right]+\left[b(x)-\langle b\rangle_{Q_{i}}\right]\right|^{r} \mathrm{~d} x\right)^{1 / r} \\
& \leq\left|Q_{i}\right|^{-1 / r}\left(\left\|b-\langle b\rangle_{Q_{j}}\right\|_{L^{r}\left(Q_{i}\right)}+\left\|b-\langle b\rangle_{Q_{i}}\right\|_{L^{r}\left(Q_{i}\right)}\right) \\
& \leq\left|Q_{i}\right|^{-1 / r}\left(\left\|b-\langle b\rangle_{Q_{j}}\right\|_{L^{r}\left(Q_{j}\right)}+\left\|b-\langle b\rangle_{Q_{i}}\right\|_{L^{r}\left(Q_{i}\right)}\right) \leq 2\left|Q_{i}\right|^{-1 / r} N .
\end{aligned}
$$

Thus, $\left(\langle b\rangle_{Q_{j}}\right)_{j}$ is a Cauchy sequence and the $\operatorname{limit} c:=\lim _{j \rightarrow \infty}\langle b\rangle_{Q_{j}}$ exists. Fatou's lemma yields the desired estimate:

$$
\|b\|_{\dot{L}^{r}\left(\mathbb{R}^{d}\right)}^{r} \leq \int_{\mathbb{R}^{d}}|b-c|^{r}=\int \lim _{j \rightarrow \infty} 1_{Q_{j}}\left|b-\langle b\rangle_{Q_{j}}\right|^{r} \leq \liminf _{j \rightarrow \infty} \int_{Q_{j}}\left|b-\langle b\rangle_{Q_{j}}\right|^{r} \leq N^{r} .
$$

For a fixed cube $Q \subset \mathbb{R}^{d}$, we have

$$
1_{Q}\left|b-\langle b\rangle_{Q}\right| \lesssim \sum_{S \in \mathscr{S}} 1_{S} f_{S}\left|b-\langle b\rangle_{S}\right|
$$


for a suitable sparse subcollection $\mathscr{S} \subset \mathscr{D}(Q)$. Here $\mathscr{D}(Q)$ consists of the dyadic subcubes of $Q$ obtained by dividing $Q$ in the natural way. For this elementary variant of Lerner's oscillation formula see e.g. [22, Lemma 3.4]. Using this we now have

$$
\left\|b-\langle b\rangle_{Q}\right\|_{L^{r}(Q)} \lesssim\left\|\sum_{S \in \mathscr{S}} 1_{S} f_{S}\left|b-\langle b\rangle_{S}\right|\right\|_{L^{r}\left(\mathbb{R}^{d}\right)} \lesssim\left(\sum_{S \in \mathscr{S}}|S|\left[f_{S}\left|b-\langle b\rangle_{S}\right|\right]^{r}\right)^{1 / r},
$$

where the last step is easily verified by dualising with $\phi \in L^{r^{\prime}}$ and using the definition of sparseness:

$$
\begin{aligned}
\int\left(\sum_{S \in \mathscr{S}} 1_{S} c_{S}\right) \phi & \lesssim \sum_{S \in \mathscr{S}} c_{S} \int_{E_{S}}\langle\phi\rangle_{S} \leq \int\left(\sum_{S \in \mathscr{S}} 1_{E(S)} c_{S}\right) M \phi \\
& \leq\left\|\sum_{S \in \mathscr{S}} 1_{E(S)} c_{S}\right\|_{L^{r}}\|M \phi\|_{L^{r^{\prime}}} \lesssim\left(\sum_{S \in \mathscr{S}}|E(S)| c_{S}^{r}\right)^{1 / r}\|\phi\|_{L^{r^{\prime}}}
\end{aligned}
$$

Dualizing the $\ell^{r}$ norm with $\ell^{r^{\prime}}$, we find that

$$
\left(\sum_{S \in \mathscr{S}}\left[|S|^{1 / r} f_{S}\left|b-\langle b\rangle_{S}\right|\right]^{r}\right)^{1 / r}=\sum_{S \in \mathscr{S}} \tilde{\lambda}_{S}|S|^{1 / r} f_{S}\left|b-\langle b\rangle_{S}\right|=\sum_{S \in \mathscr{S}} \lambda_{S} \int_{S}\left|b-\langle b\rangle_{S}\right|
$$

for suitable coefficients $\lambda_{S}$ with $\sum_{S \in \mathscr{S}}|S| \lambda_{S}^{r^{\prime}}=\sum_{S \in \mathscr{S}} \tilde{\lambda}_{S}^{r^{\prime}} \leq 1$.

Next, using sparseness and the definition of the sharp maximal operator, we observe that given an arbitrary sparse collection $\mathscr{S}$ and coefficients $\lambda_{S}$ with $\sum_{S \in \mathscr{S}}|S| \lambda_{S}^{r^{\prime}} \leq 1$, we have

$$
\begin{aligned}
\sum_{S \in \mathscr{S}}|S| \lambda_{S} f_{S}\left|b-\langle b\rangle_{S}\right| & \lesssim \sum_{S \in \mathscr{S}}|E(S)| \lambda_{S} \inf _{z \in S} M^{\#} b(z) \leq \int\left(\sum_{S \in \mathscr{S}} 1_{E(S)} \lambda_{S}\right) M^{\#} b \\
& \leq\left\|\sum_{S \in \mathscr{S}} 1_{E(S)} \lambda_{S}\right\|_{L^{r^{\prime}}}\left\|M^{\#} b\right\|_{L^{r}}=\left(\sum_{S \in \mathscr{S}}|E(S)| \lambda_{S}^{r^{\prime}}\right)^{1 / r^{\prime}}\left\|M^{\#} b\right\|_{L^{r}},
\end{aligned}
$$

where the first factor is bounded by 1 . Lastly, we have

$$
\left\|M^{\#} b\right\|_{L^{r}}=\left\|M^{\#}(b-c)\right\|_{L^{r}} \lesssim\|b-c\|_{L^{r}}
$$

for every constant $c$, and hence $\left\|M^{\#} b\right\|_{L^{r}} \lesssim\|b\|_{\dot{L}^{r}}$.

3.B. Bi-parameter spaces. For $b \in L_{\mathrm{loc}}^{1}$ and a rectangle $R=I_{1} \times I_{2}$ we denote

$$
\operatorname{osc}^{v_{1}, v_{2}}(b, R):=\left\|b-\langle b\rangle_{I_{1}, 1}-\langle b\rangle_{I_{2}, 2}+\langle b\rangle_{R}\right\|_{L_{x_{1}}^{v_{1}} L_{x_{2}}^{v_{2}}(R)}, \quad 1 \leq v_{i} \leq \infty .
$$

Homogeneous Hölder spaces. If $X$ is a Banach space with norm $|\cdot|_{X}$ and $b: \mathbb{R}^{d} \rightarrow X$, we define

$$
\|b\|_{\dot{C}^{0, \alpha}(X)}=\sup _{x \neq y} \frac{|b(x)-b(y)|_{X}}{|x-y|^{\alpha}} .
$$

In $\mathbb{R}^{d}=\mathbb{R}^{d_{1}} \times \mathbb{R}^{d_{2}}$ the natural bi-parameter homogeneous Hölder norm is

$$
\begin{aligned}
\|b\|_{\dot{C}^{0, \alpha, \beta}\left(\mathbb{R}^{d}\right)} & :=\|b\|_{\dot{C}_{x_{1}}^{0, \alpha}\left(\dot{C}_{x_{2}}^{0, \beta}\right)}=\|b\|_{\dot{C}_{x_{2}}^{0, \beta}\left(\dot{C}_{x_{1}}^{0, \alpha}\right)} \\
& =\sup _{x_{1} \neq y_{1}}\left\|\frac{b\left(x_{1}, \cdot\right)-b\left(y_{1}, \cdot\right)}{\left|x_{1}-y_{1}\right|^{\alpha}}\right\|_{\dot{C}^{0, \beta}}=\sup _{x_{2} \neq y_{2}}\left\|\frac{b\left(\cdot, x_{2}\right)-b\left(\cdot, y_{2}\right)}{\left|x_{2}-y_{2}\right|^{\beta}}\right\|_{\dot{C}^{0, \alpha}}
\end{aligned}
$$




$$
=\sup _{\substack{x_{1} \neq y_{1} \\ x_{2} \neq y_{2}}} \frac{\left|b\left(x_{1}, x_{2}\right)-b\left(x_{1}, y_{2}\right)-b\left(y_{1}, x_{2}\right)+b\left(y_{1}, y_{2}\right)\right|}{\left|x_{1}-y_{1}\right|^{\alpha}\left|x_{2}-y_{2}\right|^{\beta}} .
$$

The following oscillatory characterization holds for this norm.

3.5. Proposition. We have

$$
\|b\|_{\dot{C}^{0, \alpha, \beta}\left(\mathbb{R}^{d}\right)} \sim \sup \left\{\frac{1}{\ell\left(I_{1}\right)^{\alpha} \ell\left(I_{2}\right)^{\beta}} \frac{\operatorname{osc}^{1,1}(b, R)}{|R|}: R=I_{1} \times I_{2} \text { rectangle }\right\} .
$$

Proof. We denote the supremum on the right hand side by $N$.

" $\geq$ " is immediate, since for all $x_{i} \in I_{i}$ we have

$$
\begin{aligned}
\mid b\left(x_{1}, x_{2}\right)- & \langle b\rangle_{I_{1}, 1}\left(x_{2}\right)-\langle b\rangle_{I_{2}, 2}\left(x_{1}\right)+\langle b\rangle_{I_{1} \times I_{2}} \mid \\
& \leq f_{I_{1} \times I_{2}}\left|b\left(x_{1}, x_{2}\right)-b\left(x_{1}, y_{2}\right)-b\left(y_{1}, x_{2}\right)+b\left(y_{1}, y_{2}\right)\right| \mathrm{d} y_{1} \mathrm{~d} y_{2} \\
& \lesssim\|b\|_{\dot{C}^{0, \alpha, \beta}\left(\mathbb{R}^{d}\right)} \ell\left(I_{1}\right)^{\alpha} \ell\left(I_{2}\right)^{\beta} .
\end{aligned}
$$

For " $\lesssim$ ", notice that by Proposition 3.1 we have

$$
\begin{aligned}
\|b\|_{\dot{C}^{0, \alpha, \beta}\left(\mathbb{R}^{d}\right)} & =\sup _{x_{2} \neq y_{2}}\left\|\frac{b\left(\cdot, x_{2}\right)-b\left(\cdot, y_{2}\right)}{\left|x_{2}-y_{2}\right|^{\beta}}\right\|_{\dot{C}^{0, \alpha}} \\
& \sim \sup _{x_{2} \neq y_{2}} \frac{1}{\left|x_{2}-y_{2}\right|^{\beta}} \sup _{I_{1}} \frac{1}{\ell\left(I_{1}\right)^{\alpha}} f_{I_{1}}\left|b_{x_{2}, y_{2}}-\left\langle b_{x_{2}, y_{2}}\right\rangle_{I_{1}}\right|,
\end{aligned}
$$

where

$$
b_{x_{2}, y_{2}}=b\left(\cdot, x_{2}\right)-b\left(\cdot, y_{2}\right) .
$$

So we fix $x_{2} \neq y_{2}$ and a cube $I_{1} \subset \mathbb{R}^{d_{1}}$. By expanding $b\left(\cdot, x_{2}\right)$ as in the proof of Proposition 3.1 (using the same notation as there) we get

$$
b\left(\cdot, x_{2}\right)=\sum_{k=1}^{\infty}\left(f_{Q_{k+1}\left(x_{2}\right)} b\left(\cdot, z_{2}\right) \mathrm{d} z_{2}-f_{Q_{k}\left(x_{2}\right)} b\left(\cdot, z_{2}\right) \mathrm{d} z_{2}\right)+f_{Q\left(\frac{1}{2}\left(x_{2}+y_{2}\right), \frac{1}{2}\left|x_{2}-y_{2}\right|\right)} b\left(\cdot, z_{2}\right) \mathrm{d} z_{2} .
$$

Hence

$$
b_{x_{2}, y_{2}}=\sum_{k=1}^{\infty}\left[\langle b\rangle_{Q_{k+1}\left(x_{2}\right), 2}-\langle b\rangle_{Q_{k}\left(x_{2}\right), 2}\right]-\sum_{k=1}^{\infty}\left[\langle b\rangle_{Q_{k+1}\left(y_{2}\right), 2}-\langle b\rangle_{Q_{k}\left(y_{2}\right), 2}\right] .
$$

We first consider the contribution of the first summand. We have

$$
\begin{aligned}
& f_{I_{1}}\left|\left[\langle b\rangle_{Q_{k+1}\left(x_{2}\right), 2}-\langle b\rangle_{Q_{k}\left(x_{2}\right), 2}\right]-\left\langle\left[\langle b\rangle_{Q_{k+1}\left(x_{2}\right), 2}-\langle b\rangle_{Q_{k}\left(x_{2}\right), 2}\right]\right\rangle_{I_{1}}\right| \\
& =f_{I_{1}}\left|\left\langle b-\langle b\rangle_{I_{1}, 1}\right\rangle_{Q_{k+1}\left(x_{2}\right), 2}-\left\langle b-\langle b\rangle_{I_{1}, 1}\right\rangle_{Q_{k}\left(x_{2}\right), 2}\right| \\
& \lesssim f_{I_{1} \times Q_{k}\left(x_{2}\right)}\left|b-\langle b\rangle_{I_{1}, 1}-\left\langle b-\langle b\rangle_{I_{1}, 1}\right\rangle_{Q_{k}\left(x_{2}\right), 2}\right| \\
& \lesssim N \ell\left(I_{1}\right)^{\alpha}\left(2^{-k}\left|x_{2}-y_{2}\right|\right)^{\beta} .
\end{aligned}
$$


Thus, the contribution of the first summand to the average $f_{I_{1}}\left|b_{x_{2}, y_{2}}-\left\langle b_{x_{2}, y_{2}}\right\rangle_{I_{1}}\right|$, can be dominated by $N \ell\left(I_{1}\right)^{\alpha}\left|x_{2}-y_{2}\right|^{\beta}$. Likewise, the second summand contributes the same. Thus, we have established

$$
f_{I_{1}}\left|b_{x_{2}, y_{2}}-\left\langle b_{x_{2}, y_{2}}\right\rangle_{I_{1}}\right| \lesssim N \ell\left(I_{1}\right)^{\alpha}\left|x_{2}-y_{2}\right|^{\beta}
$$

proving that $\|b\|_{\dot{C}^{0, \alpha, \beta}\left(\mathbb{R}^{d}\right)} \lesssim N$ as desired.

For the norm

$$
\|b\|_{\dot{C}_{x_{1}}^{0, \alpha}\left(\mathrm{BMO}_{x_{2}}\right)}=\sup _{x_{1} \neq y_{1}}\left\|\frac{b\left(x_{1}, \cdot\right)-b\left(y_{1}, \cdot\right)}{\left|x_{1}-y_{1}\right|^{\beta_{1}}}\right\|_{\mathrm{BMO}\left(\mathbb{R}^{d_{2}}\right)}
$$

the following oscillatory characterization holds.

3.6. Proposition. We have

$$
\|b\|_{\dot{C}_{x_{1}}^{0, \alpha}\left(\mathrm{BMO}_{x_{2}}\right)} \sim \sup \left\{\frac{1}{\ell\left(I_{1}\right)^{\alpha}} \frac{\mathrm{Osc}^{1,1}(b, R)}{|R|}: R=I_{1} \times I_{2} \text { rectangle }\right\} .
$$

Proof. We denote the supremum on the right hand side by $N$.

We again first prove the " $\geq$ " direction. For all $x_{i} \in I_{i}$ we have

$$
\begin{aligned}
\mid b\left(x_{1}, x_{2}\right)- & \langle b\rangle_{I_{1}, 1}\left(x_{2}\right)-\langle b\rangle_{I_{2}, 2}\left(x_{1}\right)+\langle b\rangle_{I_{1} \times I_{2}} \mid \\
& =\left|f_{I_{1} \times I_{2}}\left(b\left(x_{1}, x_{2}\right)-b\left(x_{1}, y_{2}\right)-b\left(y_{1}, x_{2}\right)+b\left(y_{1}, y_{2}\right)\right) \mathrm{d} y_{1} \mathrm{~d} y_{2}\right| \\
& =\left|f_{I_{1}}\right| x_{1}-\left.y_{1}\right|^{\alpha}\left(\frac{b\left(x_{1}, x_{2}\right)-b\left(y_{1}, x_{2}\right)}{\left|x_{1}-y_{1}\right|^{\alpha}}-\left\langle\frac{b\left(x_{1}, \cdot\right)-b\left(y_{1}, \cdot\right)}{\left|x_{1}-y_{1}\right|^{\alpha}}\right\rangle_{I_{2}}\right) \mathrm{d} y_{1} \mid \\
& \lesssim \ell\left(I_{1}\right)^{\alpha} f_{I_{1}}\left|\frac{b\left(x_{1}, x_{2}\right)-b\left(y_{1}, x_{2}\right)}{\left|x_{1}-y_{1}\right|^{\alpha}}-\left\langle\frac{b\left(x_{1}, \cdot\right)-b\left(y_{1}, \cdot\right)}{\left|x_{1}-y_{1}\right|^{\alpha}}\right\rangle_{I_{2}}\right| \mathrm{d} y_{1} .
\end{aligned}
$$

Then taking the average over $I_{1} \times I_{2}$ and using Fubini we obtain the estimate as desired.

For " $\lesssim$ " we fix $x_{1}, y_{1} \in \mathbb{R}^{d_{1}}$ with $x_{1} \neq y_{1}$. Similarly as Proposition 3.5 (just let $\alpha=0$ there) we see that for all cubes $I_{2} \subset \mathbb{R}^{d_{2}}$

$$
f_{I_{2}}\left|b_{x_{1}, y_{1}}-\left\langle b_{x_{1}, y_{1}}\right\rangle_{I_{2}}\right| \lesssim N\left|x_{1}-y_{1}\right|^{\alpha}
$$

where $b_{x_{1}, y_{1}}=b\left(x_{1}, \cdot\right)-b\left(y_{1}, \cdot\right)$. This proves the claim.

The final $\dot{C}^{0, \alpha}(X)$ type space of interest to us is the bi-parameter space $\dot{C}_{x_{1}}^{0, \alpha}\left(\dot{L}_{x_{2}}^{r}\right)$.

3.7. Proposition. We have

$$
\begin{aligned}
\|b\|_{\dot{C}_{x_{1}}^{0, \alpha}\left(\dot{L}_{x_{2}}^{r}\right)} \sim \sup \left\{\frac{1}{\left|I_{1}\right| \ell\left(I_{1}\right)^{\alpha}} \sum_{S_{2} \in \mathscr{S}_{2}} \lambda_{S_{2}} \operatorname{osc}^{1,1}\left(b, I_{1} \times S_{2}\right):\right. & \\
& \left.I_{1} \subset \mathbb{R}^{d_{1}}, \mathscr{S}_{2} \text { sparse, } \sum_{S_{2} \in \mathscr{S}_{2}}\left|S_{2}\right| \lambda_{S_{2}}^{r^{\prime}} \leq 1\right\},
\end{aligned}
$$

where $I_{1} \subset \mathbb{R}^{d_{1}}$ is a cube and $\mathscr{S}_{2}$ is a sparse collection of cubes in $\mathbb{R}^{d_{2}}$. 
Proof. " $\gtrsim$ ": We apply Proposition 3.2 to the function $b\left(x_{1}, \cdot\right)-\langle b\rangle_{I_{1}, 1}$ for each $x_{1} \in I_{1}$. This shows that

$$
\begin{aligned}
\sum_{S_{2} \in \mathscr{S}_{2}} \lambda_{S_{2}} & \int_{S_{2}}\left|b\left(x_{1}, \cdot\right)-\langle b\rangle_{I_{1}, 1}-\langle b\rangle_{S_{2}, 2}\left(x_{1}\right)+\langle b\rangle_{I_{1} \times S_{2}}\right| \\
& \lesssim\left\|b\left(x_{1}, \cdot\right)-\langle b\rangle_{I_{1}, 1}\right\|_{\dot{L}_{x_{2}}^{r}} \lesssim f_{I_{1}}\left\|b\left(x_{1}, \cdot\right)-b\left(y_{1}, \cdot\right)\right\|_{\dot{L}_{x_{2}}^{r}} \mathrm{~d} y_{1} \lesssim\|b\|_{\dot{C}_{x_{1}}^{0, \alpha}\left(\dot{L}_{x_{2}}^{r}\right)} \ell\left(I_{1}\right)^{\alpha} .
\end{aligned}
$$

The penultimate estimate is easy to see using Proposition 3.2. Integrating over $x_{1} \in I_{1}$, we deduce the claimed bound.

" $\lesssim$ ": Denoting by $N$ the right-hand side, we need to prove that $\left\|b\left(x_{1}, \cdot\right)-b\left(y_{1}, \cdot\right)\right\|_{\dot{L}_{x_{2}}^{r}} \lesssim$ $N\left|x_{1}-y_{1}\right|^{\alpha}$ for all $x_{1} \neq y_{1} \in \mathbb{R}^{d_{1}}$. By Proposition 3.2, this is equivalent to

$$
\sum_{S_{2} \in \mathscr{S}_{2}} \lambda_{S_{2}} \int_{S_{2}}\left|b\left(x_{1}, \cdot\right)-b\left(y_{1}, \cdot\right)-\left\langle b\left(x_{1}, \cdot\right)-b\left(y_{1}, \cdot\right)\right\rangle_{S_{2}}\right| \lesssim N\left|x_{1}-y_{1}\right|^{\alpha},
$$

where $\mathscr{S}_{2}$ and $\lambda_{S_{2}}$ are as in the claim. We then expand $b\left(x_{1}, \cdot\right)$ as in the proof of Proposition 3.1 (using the same notation as there):

$b\left(x_{1}, \cdot\right)=\sum_{k=1}^{\infty}\left(f_{Q_{k+1}\left(x_{1}\right)} b\left(z_{1}, \cdot\right) \mathrm{d} z_{1}-f_{Q_{k}\left(x_{1}\right)} b\left(z_{1}, \cdot\right) \mathrm{d} z_{1}\right)+f_{Q\left(\frac{1}{2}\left(x_{1}+y_{1}\right), \frac{1}{2}\left|x_{1}-y_{1}\right|\right)} b\left(z_{1}, \cdot\right) \mathrm{d} z_{1}$.

Doing the same with $b\left(y_{1}, \cdot\right)$ and using the triangle inequality, we find that

LHS

$$
\begin{aligned}
& \leq \sum_{u_{1}=x_{1}, y_{1}} \sum_{k=1}^{\infty} \sum_{S_{2} \in \mathscr{S}_{2}} \lambda_{S_{2}} \int_{S_{2}}\left|\langle b\rangle_{Q_{k+1}\left(u_{1}\right), 1}-\langle b\rangle_{Q_{k}\left(u_{1}\right), 1}-\left(\langle b\rangle_{Q_{k+1}\left(u_{1}\right) \times S_{2}}-\langle b\rangle_{Q_{k}\left(u_{1}\right) \times S_{2}}\right)\right| \\
& \leq \sum_{u_{1}=x_{1}, y_{1}} \sum_{k=1}^{\infty} \sum_{S_{2} \in \mathscr{I}_{2}} \lambda_{S_{2}} \int_{S_{2}} f_{Q_{k+1}\left(u_{1}\right)}\left|b-\langle b\rangle_{Q_{k}\left(u_{1}\right), 1}-\left(\langle b\rangle_{S_{2}, 2}-\langle b\rangle_{Q_{k}\left(u_{1}\right) \times S_{2}}\right)\right| \\
& \lesssim \sum_{u_{1}=x_{1}, y_{1}} \sum_{k=1}^{\infty} \sum_{S_{2} \in \mathscr{S}_{2}} \lambda_{S_{2}} \int_{S_{2}} f_{Q_{k}\left(u_{1}\right)}\left|b-\langle b\rangle_{Q_{k}\left(u_{1}\right), 1}-\langle b\rangle_{S_{2}, 2}+\langle b\rangle_{Q_{k}\left(u_{1}\right) \times S_{2}}\right| \\
& \leq \sum_{u_{1}=x_{1}, y_{1}} \sum_{k=1}^{\infty} N \ell\left(Q_{k}\left(u_{1}\right)\right)^{\alpha} \lesssim \sum_{u_{1}=x_{1}, y_{1}} \sum_{k=1}^{\infty} N\left(2^{-k}\left|x_{1}-y_{1}\right|\right)^{\alpha} \lesssim N\left|x_{1}-y_{1}\right|^{\alpha},
\end{aligned}
$$

and this proves 3.8 .

3.9. Remark. Note that it seems somewhat important that in the above proof we apply Proposition 3.2 directly to the defining condition $\left\|b\left(x_{1}, \cdot\right)-b\left(y_{1}, \cdot\right)\right\|_{\dot{L}_{x_{2}}^{r}} \lesssim\left|x_{1}-y_{1}\right|^{\alpha}$, so that the extremizing $\mathscr{S}_{2}$ and $\lambda_{S_{2}}$ depend on $x_{1}, y_{1}$ only, and only then apply the (considerations of) Proposition 3.1. Alternatively, it might occur to one to start with (a vector-valued version of) Proposition 3.1 , reducing the proof to estimating $f_{I_{1}}\left\|b\left(z_{1}, \cdot\right)-\langle b\rangle_{I_{1}, 1}\right\|_{\dot{L}_{x_{2}}^{r}} \mathrm{~d} z_{1} \lesssim$ $\ell\left(I_{1}\right)^{\alpha}$. If we now tried to continue with Proposition 3.2. applied to $b\left(z_{1}, \cdot\right)-\langle b\rangle_{I_{1}, 1}$ for each $z_{1} \in I_{i}$, the resulting $\mathscr{S}_{2}$ and $\lambda_{S_{2}}$ would in general depend on the integration parameter $z_{1} \in I_{1}$. 
Bounded mean oscillation. We define, for $1 \leq p_{i}<\infty$, the rectangular BMO norm

$$
\|b\|_{\mathrm{BMO}_{\text {rect }}\left(p_{1}, p_{2}\right)}=\sup _{R=I_{1} \times I_{2}} \frac{\operatorname{osc}^{p_{1}, p_{2}}(b, R)}{\left|I_{1}\right|^{1 / p_{1}}\left|I_{2}\right|^{1 / p_{2}}} .
$$

This space is directly in the oscillatory form. It does not appear to enjoy the JohnNirenberg property, and so the choice of the exponents can matter. Usually in the literature

$$
\|b\|_{\mathrm{BMO}_{\text {rect }}}:=\|b\|_{\mathrm{BMO}_{\text {rect }}(2,2)} \text {. }
$$

For many purposes this is not the correct or optimal bi-parameter BMO space.

We will later have use for the smaller product BMO space, but it does not enjoy an oscillatory characterisation, and we will not discuss its definition now.

If $X$ is a Banach space, we say that a locally integrable $b: \mathbb{R}^{d} \rightarrow X$ belongs to the $X$-valued $\mathrm{BMO}$ space $\operatorname{BMO}(p, X), 0<p<\infty$, if

$$
\|b\|_{\mathrm{BMO}(p, X)}:=\sup _{I}\left(f_{I}\left|b-\langle b\rangle_{I}\right|_{X}^{p}\right)^{1 / p}<\infty .
$$

By the Banach-valued John-Nirenberg theorem (see e.g. [24, Theorem 3.2.30]) we have that all these norms are equivalent when $p$ varies, and we may again set

$$
\|b\|_{\mathrm{BMO}(X)}:=\sup _{I} f_{I}\left|b-\langle b\rangle_{I}\right|_{X} .
$$

From this point on, we do not obtain oscillatory characterization for our spaces, but we can record one-sided estimates of oscillatory quantities by function space norms. These are of philosophical use. Later, we may obtain commutator lower bounds with respect to these potentially smaller oscillatory quantities. We start with the bi-parameter space $\mathrm{BMO}_{x_{1}}\left(\dot{L}_{x_{2}}^{r}\right)$.

\subsection{Lemma. We have}

$$
\begin{aligned}
\sup \left\{\frac{1}{\left|I_{1}\right|} \sum_{S_{2} \in \mathscr{S}_{2}} \lambda_{S_{2}} \operatorname{osc}^{1,1}\left(b, I_{1} \times S_{2}\right): I_{1} \subset \mathbb{R}^{d_{1}}, \mathscr{S}_{2}\right. \text { sparse } & \left.\sum_{S_{2} \in \mathscr{S}_{2}}\left|S_{2}\right| \lambda_{S_{2}}^{r^{\prime}} \leq 1\right\} \\
& \lesssim\|b\|_{\mathrm{BMO}_{x_{1}}\left(\dot{L}_{x_{2}}^{r}\right)}
\end{aligned}
$$

where $I_{1} \subset \mathbb{R}^{d_{1}}$ is a cube and $\mathscr{S}_{2}$ is a sparse collection of cubes in $\mathbb{R}^{d_{2}}$.

Proof. As in Proposition 3.7 we have by applying Proposition 3.2 to $b\left(x_{1}, \cdot\right)-\langle b\rangle_{I_{1}, 1}$ that

$$
\begin{aligned}
f_{I_{1}} \sum_{S_{2} \in \mathscr{S}_{2}} \lambda_{S_{2}} \int_{S_{2}} \mid b\left(x_{1}, \cdot\right)- & \langle b\rangle_{I_{1}, 1}-\langle b\rangle_{S_{2}, 2}\left(x_{1}\right)+\langle b\rangle_{I_{1} \times S_{2}} \mid \\
& \lesssim f_{I_{1}}\left\|b\left(x_{1}, \cdot\right)-\langle b\rangle_{I_{1}, 1}\right\|_{\dot{L}_{x_{2}}^{r}} \lesssim\|b\|_{\mathrm{BMO}_{x_{1}}\left(\dot{L}_{x_{2}}^{r}\right)} .
\end{aligned}
$$

The space $\dot{L}^{r_{1}} \dot{L}^{r_{2}}$. The final condition that comes up in our commutator lower bounds is

$$
N:=\sup \sum_{S_{1} \in \mathscr{S}_{1}} \sum_{S_{2} \in \mathscr{S}_{2}} \lambda_{1, S_{1}} \lambda_{2, S_{2}} \operatorname{osc}^{1,1}\left(b, S_{1} \times S_{2}\right),
$$


where $r_{i} \in(1, \infty)$ and the supremum is taken over all sparse collections $\mathscr{S}_{i}$ of cubes in $\mathbb{R}^{d_{i}}$ and non-negative coefficients $\lambda_{i, S_{i}}$ satisfying $\sum_{S_{i} \in \mathscr{S}_{i}} \lambda_{i, S_{i}}^{r_{i}^{\prime}}\left|S_{i}\right| \leq 1$. We again only have the direction that

$$
N \lesssim\|b\|_{\dot{L}_{x_{1}}^{r_{1}} \dot{L}_{x_{2}}^{r_{2}}}
$$

which is primarily of philosophical use. This can be seen by utilising Proposition 3.2 and its proof.

\section{WEAK FACTORIZATION AND COROLLARIES}

4.1. Definition (Non-degenerate kernels). Let $K_{i}$ be a Calderón-Zygmund kernel on $\mathbb{R}^{d_{i}}$. We say that $K_{i}$ is (symmetrically) non-degenerate if there is a constant $c_{0}>0$ such that for every $y_{i} \in \mathbb{R}^{d_{i}}$ and $r>0$ there exists $x_{i} \in B\left(y_{i}, r\right)^{c}$ so that there holds

$$
\left|K_{i}\left(x_{i}, y_{i}\right)\right| \geq \frac{1}{c_{0} r^{d_{i}}} \quad\left(\text { and } \quad\left|K_{i}\left(y_{i}, x_{i}\right)\right| \geq \frac{1}{c_{0} r^{d_{i}}}\right) .
$$

Notice that the $x_{i}$ in the above definition necessarily satisfies $\left|x_{i}-y_{i}\right| \sim r$. If $T_{i}$ is a CZO whose kernel $K_{i}$ is a non-degenerate Calderón-Zygmund kernel, then we say that $T_{i}$ is a non-degenerate CZO. However, in what follows we do not really need CZOs, we only need the kernels, and $T_{1}$ and $T_{1}^{*}$ will just be notation for the integrals

$$
\begin{array}{ll}
T_{1} h\left(x_{1}\right)=\int_{\mathbb{R}^{d_{1}}} K_{1}\left(x_{1}, y_{1}\right) h\left(y_{1}\right) \mathrm{d} y_{1}, & x_{1} \in \mathbb{R}^{d_{1}} \backslash \operatorname{spt} h, \\
T_{1}^{*} h\left(x_{1}\right)=\int_{\mathbb{R}^{d_{1}}} K_{1}\left(y_{1}, x_{1}\right) h\left(y_{1}\right) \mathrm{d} y_{1}, & x_{1} \in \mathbb{R}^{d_{1}} \backslash \operatorname{spt} h,
\end{array}
$$

and similarly for $T_{2}$.

4.2. Definition (The "reflected" cube $\widetilde{I}_{i}$ ). Let $K_{i}$ be a fixed non-degenerate CalderónZygmund kernel on $\mathbb{R}^{d_{i}}$, and fix a large constant $A \geq 3$. For each cube $I_{i} \subset \mathbb{R}^{d_{i}}$ with center $c_{I_{i}}$ and sidelength $\ell\left(I_{i}\right)$, we define another cube $\widetilde{I}_{i} \subset \mathbb{R}^{d_{i}}$ of the same size by choosing a center $c_{\widetilde{I}_{i}}$, guaranteed by the non-degenaracy of $K_{i}$, so that

$$
\left|c_{I_{i}}-c_{\widetilde{I}_{i}}\right| \sim A \ell\left(I_{i}\right), \quad\left|K_{i}\left(c_{\widetilde{I}_{i}}, c_{I_{i}}\right)\right| \sim\left(A \ell\left(I_{i}\right)\right)^{-d_{i}} .
$$

Notice that $\operatorname{dist}\left(I_{i}, \widetilde{I}_{i}\right) \sim A \ell\left(I_{i}\right)$. If $K_{i}$ is symmetrically non-degenerate, we require in addition that $\left|K_{i}\left(c_{I_{i}}, c_{\widetilde{I}_{i}}\right)\right| \sim\left(A \ell\left(I_{i}\right)\right)^{-d_{i}}$. While the choice of $\widetilde{I}_{i}$ may not be unique in general, in the symmetric case we can and will make it in such a way that $\widetilde{\widetilde{I}}_{i}=I_{i}$.

By (the proof) of Proposition 2.2 in [22] we have for all $x_{i} \in \widetilde{I}_{i}$ and $y_{i} \in I_{i}$ that

$$
\left|K_{i}\left(x_{i}, y_{i}\right)-K_{i}\left(c_{\widetilde{I}_{i}}, c_{I_{i}}\right)\right| \leq \frac{C A^{-\alpha_{i}}}{\left(A \ell\left(I_{i}\right)\right)^{d_{i}}} .
$$

If $K_{1}$ and $K_{2}$ are two (symmetrically) non-degenerate Calderón-Zygmund kernels on $\mathbb{R}^{d_{1}}$ and $\mathbb{R}^{d_{2}}$, and $I_{i} \subset \mathbb{R}^{d_{i}}$ are cubes, we of course define $\widetilde{I}_{i}$ with respect to $K_{i}$. For each rectangle $R=I_{1} \times I_{2} \subset \mathbb{R}^{d}=\mathbb{R}^{d_{1}} \times \mathbb{R}^{d_{2}}$, we define $\widetilde{R}:=\widetilde{I}_{1} \times \widetilde{I}_{2}$.

For a rectangle $R=I_{1} \times I_{2} \subset \mathbb{R}^{d_{1}+d_{2}}=\mathbb{R}^{d}$ and $p_{1}, p_{2} \in[1, \infty]$ we denote

$$
L_{x_{1}, 0}^{p_{1}} L_{x_{2}, 0}^{p_{2}}(R)=\left\{f \in L_{x_{1}}^{p_{1}} L_{x_{2}}^{p_{2}}(R): \int_{I_{1}} f=\int_{I_{2}} f=0\right\} .
$$


We record and prove the following bi-parameter weak factorization theorem. A simpler one-parameter version is Lemma 2.5 of [22]. This will have important corollaries that we use to deduce the commutator lower bounds.

4.4. Lemma. Let $K_{i}$ be a non-degenerate kernel on $\mathbb{R}^{d_{i}}, i=1,2$. If $R=I_{1} \times I_{2} \subset \mathbb{R}^{d}$ is a rectangle and $f \in L_{00}^{1}(R)$, then we can decompose

$$
f=1_{\widetilde{R}} T_{1} T_{2} h-T_{2} h \cdot T_{1}^{*} 1_{\widetilde{R}}-T_{1} h \cdot T_{2}^{*} 1_{\widetilde{R}}+h T_{1}^{*} T_{2}^{*} 1_{\widetilde{R}}+\sum_{j=1}^{3} \tilde{f}_{j},
$$

where each $\tilde{f}_{j} \in L_{00}^{1}\left(R_{j}\right)$ for $R_{1}=\widetilde{I}_{1} \times I_{2}, R_{2}=I_{1} \times \widetilde{I}_{2}$ and $R_{3}=\widetilde{R}$, and they satisfy

$$
\left|\tilde{f}_{1}(x)\right| \lesssim A^{-\alpha_{1}} 1_{\widetilde{I}_{1}}\left(x_{1}\right)\langle|f|\rangle_{I_{1}, 1}\left(x_{2}\right), \quad\left|\tilde{f}_{2}(x)\right| \lesssim A^{-\alpha_{2}}\langle|f|\rangle_{I_{2}, 2}\left(x_{1}\right) 1_{\widetilde{I}_{2}}\left(x_{2}\right)
$$

and

$$
\left|\tilde{f}_{3}(x)\right| \lesssim A^{-\alpha_{1}} A^{-\alpha_{2}} 1_{\widetilde{R}}(x)\langle|f|\rangle_{R}
$$

Moreover, $h \in L^{1}(R)$ satisfies

$$
|h(x)| \lesssim A^{d}|f(x)|
$$

In particular, if $f \in L_{x_{1}, 0}^{u_{1}} L_{x_{2}, 0}^{u_{2}}(R), 1 \leq u_{i} \leq \infty$, then also

$$
\|h\|_{L_{x_{1}}^{u_{1}} L_{x_{2}}^{u_{2}}} \lesssim A^{d}\|f\|_{L_{x_{1}}^{u_{1}} L_{x_{2}}^{u_{2}}} \quad \text { and } \quad\left\|\tilde{f}_{j}\right\|_{L_{x_{1}}^{u_{1}} L_{x_{2}}^{u_{2}}} \lesssim A^{-\min \left(\alpha_{1}, \alpha_{2}\right)}\|f\|_{L_{x_{1}}^{u_{1}} L_{x_{2}}^{u_{2}}},
$$

where the implicit constants depend only on the dimensions and the kernel constants.

Proof. Denote $\phi=1_{\widetilde{R}}=1_{\widetilde{I}_{1}} \otimes 1_{\widetilde{I}_{2}}=: \phi_{1} \otimes \phi_{2}$. We write

$$
f=\frac{f}{T_{1}^{*} T_{2}^{*} \phi} T_{1}^{*} T_{2}^{*} \phi=\phi T_{1} T_{2} h-T_{2} h \cdot T_{1}^{*} \phi-T_{1} h \cdot T_{2}^{*} \phi+h T_{1}^{*} T_{2}^{*} \phi+\tilde{f},
$$

where

$$
h:=\frac{f}{T_{1}^{*} T_{2}^{*} \phi}
$$

and

$$
\begin{aligned}
\tilde{f} & =T_{1} h \cdot T_{2}^{*} \phi+T_{2} h \cdot T_{1}^{*} \phi-\phi T_{1} T_{2} h \\
& =\phi_{1} T_{1}\left(\frac{f}{T_{1}^{*} \phi_{1}}\right)+\phi_{2} T_{2}\left(\frac{f}{T_{2}^{*} \phi_{2}}\right)-\phi T_{1}\left(\frac{1}{T_{1}^{*} \phi_{1}} T_{2}\left(\frac{f}{T_{2}^{*} \phi_{2}}\right)\right) \\
& =\tilde{f}_{1}+\tilde{f}_{2}+\tilde{f}_{3} .
\end{aligned}
$$

Everything is well-defined, since in fact we have the estimates $T_{i}^{*} 1_{\widetilde{I}_{i}}\left(x_{i}\right) \gtrsim A^{-d_{i}}$ for $x \in I_{i}$ (remember that $f$ is supported on $R$ ). To see this, fix $x_{1} \in I_{1}$ and write

$$
\begin{aligned}
T_{1}^{*} 1_{\widetilde{I}_{1}}\left(x_{1}\right) & =\int_{\widetilde{I}_{1}} K_{1}\left(y_{1}, x_{1}\right) \mathrm{d} y_{1} \\
& =K_{1}\left(c_{\widetilde{I}_{1}}, c_{I_{1}}\right)\left|I_{1}\right|+\int_{\widetilde{I}_{1}}\left(K_{1}\left(y_{1}, x_{1}\right)-K_{1}\left(c_{\widetilde{I}_{1}}, c_{I_{1}}\right)\right) \mathrm{d} y_{1} .
\end{aligned}
$$

Using (4.3) it is then easy to see that $\left|T_{1}^{*} 1_{\widetilde{I}_{1}}\left(x_{1}\right)\right| \gtrsim A^{-d_{1}}$. The other estimate is symmetric. This also gives that

$$
|h(x)| \lesssim A^{d}|f(x)|
$$


It is also immediate that $\int_{I_{2}} \tilde{f}_{1}=0$ and

$$
\int_{\widetilde{I}_{1}} \tilde{f}_{1}=\int_{\mathbb{R}^{d_{1}}} \phi_{1} T_{1}\left(\frac{f}{T_{1}^{*} \phi_{1}}\right)=\int_{\mathbb{R}^{d_{1}}} T_{1}^{*} \phi_{1} \frac{f}{T_{1}^{*} \phi_{1}}=\int_{I_{1}} f=0 .
$$

Similarly, we can see that $\int_{I_{1}} \tilde{f}_{2}=\int_{\widetilde{I}_{2}} \tilde{f}_{2}=\int_{\widetilde{I}_{1}} \tilde{f}_{3}=\int_{\widetilde{I}_{2}} \tilde{f}_{3}=0$.

We now prove the poinwise estimates of $\tilde{f}_{i}$. Let $g: \mathbb{R}^{d} \rightarrow \mathbb{C}$ satisfy $g(x)=0$ if $x_{1} \notin I_{1}$ and $\int_{I_{1}} g=0$. For $x=\left(x_{1}, x_{2}\right) \in \widetilde{I}_{1} \times \mathbb{R}^{d_{2}}$ we write

$$
T_{1}\left(\frac{g}{T_{1}^{*} \phi_{1}}\right)(x)=T_{1}\left(\frac{g\left(\cdot, x_{2}\right)}{T_{1}^{*} \phi_{1}}-\frac{g\left(\cdot, x_{2}\right)}{\left|I_{1}\right| K_{1}\left(c_{\widetilde{I}_{1}}, c_{I_{1}}\right)}\right)\left(x_{1}\right)+\frac{1}{\left|I_{1}\right| K_{1}\left(c_{\widetilde{I}_{1}}, c_{I_{1}}\right)} T_{1}\left(g\left(\cdot, x_{2}\right)\right)\left(x_{1}\right) .
$$

We have using (4.3) that

$$
\begin{aligned}
\left|\frac{1}{\left|I_{1}\right| K_{1}\left(c_{\widetilde{I}_{1}}, c_{I_{1}}\right)} T_{1}\left(g\left(\cdot, x_{2}\right)\right)\left(x_{1}\right)\right| & \sim A^{d_{1}}\left|\int_{I_{1}}\left[K_{1}\left(x_{1}, y_{1}\right)-K_{1}\left(c_{\widetilde{I}_{1}}, c_{I_{1}}\right)\right] g\left(y_{1}, x_{2}\right) \mathrm{d} y_{1}\right| \\
& \lesssim A^{-\alpha_{1}} f_{I_{1}}\left|g\left(\cdot, x_{2}\right)\right| .
\end{aligned}
$$

It is similarly straightforward that

$$
\left|T_{1}\left(\frac{g\left(\cdot, x_{2}\right)}{T_{1}^{*} \phi_{1}}-\frac{g\left(\cdot, x_{2}\right)}{\left|I_{1}\right| K_{1}\left(c_{\widetilde{I}_{1}}, c_{I_{1}}\right)}\right)\left(x_{1}\right)\right| \lesssim A^{-\alpha_{1}} f_{I_{1}}\left|g\left(\cdot, x_{2}\right)\right| .
$$

It follows that

$$
\left|T_{1}\left(\frac{g}{T_{1}^{*} \phi_{1}}\right)(x)\right| \lesssim A^{-\alpha_{1}} f_{I_{1}}\left|g\left(\cdot, x_{2}\right)\right|, \quad x \in \widetilde{I}_{1} \times \mathbb{R}^{d_{2}} .
$$

Applying this to $g=f$ we get

$$
\left|\tilde{f}_{1}(x)\right| \lesssim A^{-\alpha_{1}} 1_{\widetilde{I}_{1}}\left(x_{1}\right)\langle|f|\rangle_{I_{1}, 1}\left(x_{2}\right) .
$$

The estimate

$$
\left|\tilde{f}_{2}(x)\right| \lesssim A^{-\alpha_{2}}\langle|f|\rangle_{I_{2}, 2}\left(x_{1}\right) 1_{\widetilde{I}_{2}}\left(x_{2}\right)
$$

is symmetric. Lastly, notice that

$$
\tilde{f}_{3}=\phi_{1} T_{1}\left(\frac{1}{T_{1}^{*} \phi_{1}} \phi_{2} T_{2}\left(\frac{f}{T_{2}^{*} \phi_{2}}\right)\right)=\phi_{1} T_{1}\left(\frac{\tilde{f}_{2}}{T_{1}^{*} \phi_{1}}\right)
$$

so that applying (4.5) to $g=\tilde{f}_{2}$ (which is a legitimate choice) and the estimate of $\tilde{f}_{2}$, we get that for $x \in \widetilde{I}_{1} \times \widetilde{I}_{2}$ there holds

$$
\left|\tilde{f}_{3}(x)\right| \lesssim A^{-\alpha_{1}} f_{I_{1}}\left|\tilde{f}_{2}\left(y_{1}, x_{2}\right)\right| \mathrm{d} y_{1} \lesssim A^{-\alpha_{1}} A^{-\alpha_{2}} f_{R}|f|
$$

The claimed $L^{u_{1}} L^{u_{2}}$ estimates are clear from the pointwise estimates.

4.6. Remark. A much weaker modulus of continuity like in [22] for the kernels $K_{1}, K_{2}$ would also work, but we prefer to use the standard estimates here for simplicity. The weaker assumption for $K_{1}$ would be

$$
\left|K_{1}\left(x_{1}, y_{1}\right)-K_{1}\left(x_{1}^{\prime}, y_{1}\right)\right|+\left|K_{1}\left(y_{1}, x_{1}\right)-K_{1}\left(y_{1}, x_{1}^{\prime}\right)\right| \leq \frac{1}{\left|x_{1}-y_{1}\right| d_{1}} \omega\left(\frac{\left|x_{1}-x_{1}^{\prime}\right|}{\left|x_{1}-y_{1}\right|}\right)
$$


whenever $\left|x_{1}-x_{1}^{\prime}\right| \leq\left|x_{1}-y_{1}\right| / 2$. In these lower bound arguments it is only needed that the increasing modulus of continuity $\omega:[0,1) \rightarrow[0, \infty)$ satisfies $\omega(t) \rightarrow 0$ when $t \rightarrow 0$.

The previous lemma will be used to control the oscillations (3.4) via the following basic observation. The idea is to decompose the dualizing $f$ like above.

4.7. Lemma. For $v_{1}, v_{2} \in[1, \infty]$, we have

$$
\operatorname{osc}^{v_{1}, v_{2}}(b, R) \sim \sup \left\{\left|\int_{R} b f\right|:\|f\|_{L_{x_{1}, 0}^{v_{1}^{\prime}} L_{x_{2}, 0}^{v_{2}^{\prime}(R)}} \leq 1\right\} .
$$

Proof. By standard dualities, we have

$$
\begin{aligned}
\operatorname{Osc}^{v_{1}, v_{2}}(b, R) & =\sup \left\{\left|\iint_{I_{1} \times I_{2}}\left[b-\langle b\rangle_{I_{1}, 1}-\langle b\rangle_{I_{2}, 2}+\langle b\rangle_{R}\right] g\right|:\|g\|_{L^{v_{1}^{\prime}} L^{v_{2}^{\prime}(R)}} \leq 1\right\} \\
& =\sup \left\{\left|\iint_{I_{1} \times I_{2}} b\left[g-\langle g\rangle_{I_{1}, 1}-\langle g\rangle_{I_{2}, 2}+\langle g\rangle_{R}\right]\right|:\|g\|_{L^{v_{1}^{\prime}} L^{v_{2}^{\prime}(R)}} \leq 1\right\} \\
& \leq \sup \left\{\left|\iint_{I_{1} \times I_{2}} b g\right|:\|g\|_{L_{0}^{v_{1}^{\prime}} L_{0}^{v_{2}^{\prime}}(R)} \leq 4\right\} \\
& =\sup \left\{\left|\iint_{I_{1} \times I_{2}}\left[b-\langle b\rangle_{I_{1}, 1}-\langle b\rangle_{I_{2}, 2}+\langle b\rangle_{R}\right] g\right|:\|g\|_{L_{0}^{v_{1}^{\prime}} L_{0}^{v_{2}^{\prime}(R)}} \leq 4\right\} \\
& \leq 4 \operatorname{osc}^{v_{1}, v_{2}}(b, R),
\end{aligned}
$$

so that all these quantities are actually comparable.

Motivated by this we define the following truncated oscillation, which is finite even when $b \in L_{\text {loc }}^{1}$. This is important for technical reasons - the absorption argument below requires a finite quantity. For $b \in L_{\text {loc }}^{1}$ and $N \in \mathbb{N}$ we define

$$
\operatorname{osc}_{N}^{v_{1}, v_{2}}(b, R):=\sup \left\{\left|\iint_{R} b f\right|:\|f\|_{L_{0}^{v_{1}^{\prime}} L_{0}^{v_{2}^{\prime}(R)}} \leq 1 \text { and }\|f\|_{L^{\infty}} \leq N\right\}<\infty .
$$

4.8. Remark. When $v_{1}=v_{2}=1$ - which is the case of most interest - we can directly work with $\operatorname{osc}^{1,1}(b, R) \sim \operatorname{osc}_{N}^{1,1}(b, R)$.

4.9. Lemma. Let $K_{i}$ be a non-degenerate kernel on $\mathbb{R}^{d_{i}}, i=1$, 2. If we fix $A \geq 3$ in Definition 4.2 large enough (depending on the dimensions and kernel constants), then for each $b \in L_{\text {loc' }}^{1}$ $1 \leq v_{i} \leq \infty, N \in \mathbb{N}$ and rectangle $R=I_{1} \times I_{2} \subset \mathbb{R}^{d}$ we have

$$
\begin{aligned}
\operatorname{osc}_{N}^{v_{1}, v_{2}}(b, R) \lesssim & \left|\left\langle\left[T_{1},\left[b, T_{2}\right]\right] h_{0}, 1_{R_{3}}\right\rangle\right|+\left|\left\langle\left[T_{1}^{*},\left[b, T_{2}\right]\right] h_{1}, 1_{R_{2}}\right\rangle\right| \\
& +\left|\left\langle\left[T_{1},\left[b, T_{2}^{*}\right]\right] h_{2}, 1_{R_{1}}\right\rangle\right|+\left|\left\langle\left[T_{1}^{*},\left[b, T_{2}^{*}\right]\right] h_{3}, 1_{R_{0}}\right\rangle\right|
\end{aligned}
$$

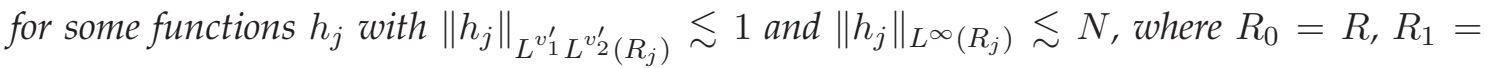
$\widetilde{I}_{1} \times I_{2}, R_{2}=I_{1} \times \widetilde{I}_{2}, R_{3}=\widetilde{R}$.

If $K_{1}$ is symmetrically non-degenerate, we get

$$
\begin{aligned}
\operatorname{osc}_{N}^{v_{1}, v_{2}}(b, R) \lesssim & \left|\left\langle\left[T_{1},\left[b, T_{2}\right]\right] h_{0}, 1_{R_{3}}\right\rangle\right|+\left|\left\langle\left[T_{1},\left[b, T_{2}\right]\right] h_{1}, 1_{R_{2}}\right\rangle\right| \\
& +\left|\left\langle\left[T_{1}^{*},\left[b, T_{2}^{*}\right]\right] h_{2}, 1_{R_{1}}\right\rangle\right|+\left|\left\langle\left[T_{1}^{*},\left[b, T_{2}^{*}\right]\right] h_{3}, 1_{R_{0}}\right\rangle\right| .
\end{aligned}
$$


A symmetric estimate holds if $K_{2}$ is symmetrically non-degenerate. If both $K_{1}$ and $K_{2}$ are symmetrically non-degenerate, then

$$
\operatorname{Osc}_{N}^{v_{1}, v_{2}}(b, R) \lesssim \sum_{j=0}^{3}\left|\left\langle\left[T_{1},\left[b, T_{2}\right]\right] h_{j}, 1_{\widetilde{R}_{j}}\right\rangle\right| \cdot
$$

Proof. Let $N$ be fixed. Fix $f \in L_{0}^{v_{1}^{\prime}} L_{0}^{v_{2}^{\prime}}(R)$ so that $\|f\|_{L_{0}^{v_{1}^{\prime}} L_{0}^{v_{2}^{\prime}(R)}} \leq 1,\|f\|_{L^{\infty}} \leq N$ and $\operatorname{osc}_{N}^{v_{1}, v_{2}}(b, R) \lesssim|\langle b, f\rangle|$. We expand this $f$ according to Lemma 4.4 to arrive at

$$
\begin{aligned}
\langle b, f\rangle & =\left\langle b, 1_{\widetilde{R}} T_{1} T_{2} h_{0}-T_{2} h_{0} \cdot T_{1}^{*} 1_{\widetilde{R}}-T_{1} h_{0} \cdot T_{2}^{*} 1_{\widetilde{R}}+h_{0} T_{1}^{*} T_{2}^{*} 1_{\widetilde{R}}\right\rangle+\sum_{j=1}^{3}\left\langle b, \tilde{f}_{j}\right\rangle \\
& =\left\langle b T_{1} T_{2} h_{0}-T_{1}\left(b T_{2} h_{0}\right)-T_{2}\left(b T_{1} h_{0}\right)+T_{1} T_{2}\left(b h_{0}\right), 1_{\widetilde{R}}\right\rangle+\sum_{j=1}^{3}\left\langle b, \tilde{f}_{j}\right\rangle \\
& =-\left\langle\left[T_{1},\left[b, T_{2}\right]\right] h_{0}, 1_{\widetilde{R}}\right\rangle+\sum_{j=1}^{3}\left\langle b, \tilde{f}_{j}\right\rangle .
\end{aligned}
$$

Hence, we have

$$
\operatorname{osc}_{N}^{v_{1}, v_{2}}(b, R) \lesssim|\langle b, f\rangle| \leq\left|\left\langle\left[T_{1},\left[b, T_{2}\right]\right] h_{0}, 1_{\widetilde{R}}\right\rangle\right|+\sum_{j=1}^{3}\left|\left\langle b, \tilde{f}_{j}\right\rangle\right|,
$$

where, by Lemma 4.4 ,

$$
\left|\left\langle b, \tilde{f}_{j}\right\rangle\right| \lesssim \operatorname{osc}_{N}^{v_{1}, v_{2}}\left(b, R_{j}\right) A^{-\min \left(\alpha_{1}, \alpha_{2}\right)}
$$

and thus

$$
\operatorname{osc}_{N}^{v_{1}, v_{2}}\left(b, R_{0}\right) \lesssim\left|\left\langle\left[T_{1},\left[b, T_{2}\right]\right] h_{0}, 1_{R_{3}}\right\rangle\right|+A^{-\min \left(\alpha_{1}, \alpha_{2}\right)} \sum_{j \in\{1,2,3\}} \operatorname{osc}_{N}^{v_{1}, v_{2}}\left(b, R_{j}\right)
$$

where $\left\|h_{0}\right\|_{L^{v_{1}^{\prime}} L_{2}^{v_{2}^{\prime}(R)}} \lesssim A^{d}$ and $\left\|h_{0}\right\|_{L^{\infty}(R)} \lesssim A^{d} N$.

Notice that if $\widetilde{Q}$ is a reflected cube of $Q$ with respect to a non-degenerate kernel $K$, then $Q$ is a reflected cube of $\widetilde{Q}$ with respect to $K^{*}$. Therefore, we also get

$$
\begin{gathered}
\operatorname{osc}_{N}^{v_{1}, v_{2}}\left(b, R_{1}\right) \lesssim\left|\left\langle\left[T_{1}^{*},\left[b, T_{2}\right]\right] h_{1}, 1_{R_{2}}\right\rangle\right|+A^{-\min \left(\alpha_{1}, \alpha_{2}\right)} \sum_{j \in\{0,2,3\}} \operatorname{osc}_{N}^{v_{1}, v_{2}}\left(b, R_{j}\right), \\
\operatorname{osc}_{N}^{v_{1}, v_{2}}\left(b, R_{2}\right) \lesssim\left|\left\langle\left[T_{1},\left[b, T_{2}^{*}\right]\right] h_{2}, 1_{R_{1}}\right\rangle\right|+A^{-\min \left(\alpha_{1}, \alpha_{2}\right)} \sum_{j \in\{0,1,3\}} \operatorname{osc}_{N}^{v_{1}, v_{2}}\left(b, R_{j}\right)
\end{gathered}
$$

and

$$
\operatorname{osc}_{N}^{v_{1}, v_{2}}\left(b, R_{3}\right) \lesssim\left|\left\langle\left[T_{1}^{*},\left[b, T_{2}^{*}\right]\right] h_{3}, 1_{R_{0}}\right\rangle\right|+A^{-\min \left(\alpha_{1}, \alpha_{2}\right)} \sum_{j \in\{0,1,2\}} \operatorname{osc}_{N}^{v_{1}, v_{2}}\left(b, R_{j}\right)
$$


Combining (4.11), (4.12), (4.13) and (4.14), and fixing $A$ large enough, we obtain by an absorption argument that

$$
\begin{aligned}
\operatorname{osc}_{N}^{v_{1}, v_{2}}(b, R) & \leq \sum_{j=0}^{3} \operatorname{osc}_{N}^{v_{1}, v_{2}}\left(b, R_{j}\right) \\
& \lesssim\left|\left\langle\left[T_{1},\left[b, T_{2}\right]\right] h_{0}, 1_{R_{3}}\right\rangle\right|+\left|\left\langle\left[T_{1}^{*},\left[b, T_{2}\right]\right] h_{1}, 1_{R_{2}}\right\rangle\right| \\
& +\left|\left\langle\left[T_{1},\left[b, T_{2}^{*}\right]\right] h_{2}, 1_{R_{1}}\right\rangle\right|+\left|\left\langle\left[T_{1}^{*},\left[b, T_{2}^{*}\right]\right] h_{3}, 1_{R_{0}}\right\rangle\right|,
\end{aligned}
$$

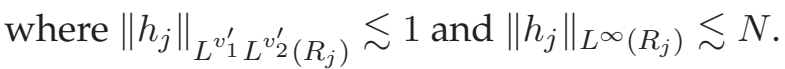

Next, if $K$ is symmetrically non-degenerate and $\widetilde{Q}$ is a reflected cube of $Q$ with respect to $K$, then $Q$ is a reflected cube of $\widetilde{Q}$ with respect to $K$ and $\widetilde{Q}$ is also a reflected cube of $Q$ with respect to $K^{*}$. Suppose $K_{1}$ is symmetrically non-degenerate. Then we can replace (4.12) by

$$
\operatorname{osc}_{N}^{v_{1}, v_{2}}\left(b, R_{1}\right) \lesssim\left|\left\langle\left[T_{1},\left[b, T_{2}\right]\right] h_{1}, 1_{R_{2}}\right\rangle\right|+A^{-\min \left(\alpha_{1}, \alpha_{2}\right)} \sum_{j \in\{0,2,3\}} \operatorname{osc}_{N}^{v_{1}, v_{2}}\left(b, R_{j}\right),
$$

and (4.13) by

$$
\operatorname{osc}_{N}^{v_{1}, v_{2}}\left(b, R_{2}\right) \lesssim\left|\left\langle\left[T_{1}^{*},\left[b, T_{2}^{*}\right]\right] h_{2}, 1_{R_{1}}\right\rangle\right|+A^{-\min \left(\alpha_{1}, \alpha_{2}\right)} \sum_{j \in\{0,1,3\}} \operatorname{osc}_{N}^{v_{1}, v_{2}}\left(b, R_{j}\right)
$$

The other symmetry statements are similar. This ends our proof.

From this point on, given non-degenerate kernels $K_{i}$, we always consider $A$ fixed large enough so that the conclusion of Lemma 4.9 hold.

4.17. Remark. We have that $\left[T_{1},\left[b, T_{2}\right]\right]^{*}=\left[T_{1}^{*},\left[b, T_{2}^{*}\right]\right]$, but a partial adjoint like $\left[T_{1}^{*},\left[b, T_{2}\right]\right]$ can more easily be unbounded even if $\left[T_{1},\left[b, T_{2}\right]\right]$ is bounded. Thus, it can be useful to be able to eliminate these partial adjoint terms if at least one of the kernels is symmetrically non-degenerate.

4.A. Off-support constants. Notice that if we take a pairing, such as $\left|\left\langle\left[T_{1},\left[b, T_{2}\right]\right] h_{0}, 1_{\tilde{R}}\right\rangle\right|$, from the conclusion of Lemma 4.9, then $h_{0}$ is supported on $I_{1} \times I_{2}$ and $\widetilde{R}=\widetilde{I}_{1} \times \widetilde{I}_{2}$ satisfies $\operatorname{dist}\left(I_{i}, \widetilde{I}_{i}\right) \sim \ell\left(I_{i}\right)$. Moreover, the case $v_{1}=v_{2}=1$ is a bit special, as then $\left\|h_{j}\right\|_{L^{\infty}\left(R_{j}\right)} \lesssim 1$.

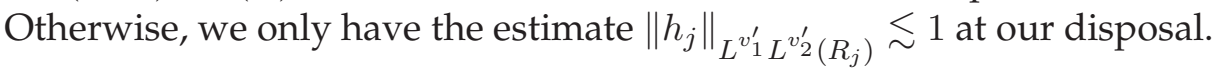

We fix $b \in L_{\text {loc }}^{1}$ and non-degenerate kernels $K_{i}$ - the off-support constants will depend on these but this will not be denoted. We first define off-support constants that are useful in the $v_{1}=v_{2}=1$ situation - which is a case that is encountered often. For $s_{1}, s_{2}, t_{1}, t_{2} \in$ $(1, \infty)$ and $B(x, y)=b\left(x_{1}, x_{2}\right)-b\left(x_{1}, y_{2}\right)-b\left(y_{1}, x_{2}\right)+b\left(y_{1}, y_{2}\right)$ define

$$
\begin{aligned}
\operatorname{Off}_{\left(s_{1}, s_{2}\right)}^{\left(t_{1}, t_{2}\right)}=\sup \frac{1}{\left|J_{1}\right|^{1+1 / s_{1}-1 / t_{1}}} & \frac{1}{\left|J_{2}\right|^{1+1 / s_{2}-1 / t_{2}}} \\
& \left|\iint_{\mathbb{R}^{d} \times \mathbb{R}^{d}} B(x, y) K_{1}\left(x_{1}, y_{1}\right) K_{2}\left(x_{2}, y_{2}\right) f_{1}(y) f_{2}(x) \mathrm{d} y \mathrm{~d} x\right|,
\end{aligned}
$$

where the supremum is taken over rectangles $P_{1}=J_{1} \times J_{2}$ and $P_{2}=L_{1} \times L_{2}$ with

$$
\ell\left(J_{i}\right)=\ell\left(L_{i}\right) \quad \text { and } \quad \operatorname{dist}\left(J_{i}, L_{i}\right) \sim \ell\left(J_{i}\right)
$$


and over functions $f_{i} \in L^{\infty}\left(P_{i}\right)$ with $\left\|f_{j}\right\|_{L^{\infty}} \leq 1$. We also denote $\mathrm{Off}_{\left(s_{1}, s_{2}\right)}^{\left(t_{1}, t_{2}\right), i *}$ the constant where $K_{i}$ is replaced by $K_{i}^{*}$.

If $T_{i}$ is a non-degenerate $\mathrm{CZO}$ with kernel $K_{i}$, then

$$
\mathrm{Off}_{\left(s_{1}, s_{2}\right)}^{\left(t_{1}, t_{2}\right)} \leq\left\|\left[T_{1},\left[b, T_{2}\right]\right]\right\|_{L_{x_{1}}^{s_{1}} L_{x_{2}}^{s_{2}} \rightarrow L_{x_{1}}^{t_{1}} L_{x_{2}}^{t_{2}}}
$$

whenever the commutator exists as a bounded linear mapping. This explains why these weaker off-support constants make sense.

4.18. Theorem. Let $K_{i}$ be a non-degenerate kernel on $\mathbb{R}^{d_{i}}, i=1,2$, and let $b \in L_{\text {loc }}^{1}$. Let $p_{1}, p_{2}, q_{1}, q_{2} \in(1, \infty)$. Then we have for all rectangles $R=I_{1} \times I_{2}$ that

$$
\frac{\operatorname{Osc}^{1,1}(b, R)}{|R|} \lesssim\left(\operatorname{Off}_{\left(p_{1}, p_{2}\right)}^{\left(q_{1}, q_{2}\right)}+\operatorname{Off}_{\left(s_{1}, s_{2}\right)}^{\left(t_{1}, t_{2}\right), 1 *}\right)\left|I_{1}\right|^{\frac{1}{p_{1}}-\frac{1}{q_{1}}}\left|I_{2}\right|^{\frac{1}{p_{2}}-\frac{1}{q_{2}}}
$$

with any $s_{i}, t_{i} \in(1, \infty)$ satisfying $1 / p_{i}-1 / q_{i}=1 / s_{i}-1 / t_{i}, i=1,2$.

If, in addition, one of the kernels $K_{i}$ is symmetrically non-degenerate, then

$$
\frac{\operatorname{osc}^{1,1}(b, R)}{|R|} \lesssim \operatorname{Off}_{\left(p_{1}, p_{2}\right)}^{\left(q_{1}, q_{2}\right)}\left|I_{1}\right|^{\frac{1}{p_{1}}-\frac{1}{q_{1}}}\left|I_{2}\right|^{\frac{1}{p_{2}}-\frac{1}{q_{2}}} .
$$

Proof. By Lemma 4.9 we have for each rectangle $R=I_{1} \times I_{2} \subset \mathbb{R}^{d}$ that

$$
\begin{aligned}
\operatorname{osc}^{1,1}(b, R) \lesssim & \left|\left\langle\left[T_{1},\left[b, T_{2}\right]\right] h_{0}, 1_{R_{3}}\right\rangle\right|+\left|\left\langle\left[T_{1}^{*},\left[b, T_{2}\right]\right] h_{1}, 1_{R_{2}}\right\rangle\right| \\
& +\left|\left\langle\left[T_{1},\left[b, T_{2}^{*}\right]\right] h_{2}, 1_{R_{1}}\right\rangle\right|+\left|\left\langle\left[T_{1}^{*},\left[b, T_{2}^{*}\right]\right] h_{3}, 1_{R_{0}}\right\rangle\right|
\end{aligned}
$$

for some functions $h_{j}$ with $\left\|h_{j}\right\|_{L^{\infty}\left(R_{j}\right)} \lesssim 1$, where $R_{0}=R, R_{1}=\widetilde{I}_{1} \times I_{2}, R_{2}=I_{1} \times \widetilde{I}_{2}$, $R_{3}=\widetilde{R}$. Clearly, we have

$$
\left|\left\langle\left[T_{1},\left[b, T_{2}\right]\right] h_{0}, 1_{R_{3}}\right\rangle\right|+\left|\left\langle\left[T_{1}^{*},\left[b, T_{2}^{*}\right]\right] h_{3}, 1_{R_{0}}\right\rangle\right| \lesssim \operatorname{Off}_{\left(p_{1}, p_{2}\right)}^{\left(q_{1}, q_{2}\right)}|R|\left|I_{1}\right|^{\frac{1}{p_{1}}-\frac{1}{q_{1}}}\left|I_{2}\right|^{\frac{1}{p_{2}}-\frac{1}{q_{2}}}
$$

and

$$
\left|\left\langle\left[T_{1}^{*},\left[b, T_{2}\right]\right] h_{1}, 1_{R_{2}}\right\rangle\right|+\left|\left\langle\left[T_{1},\left[b, T_{2}^{*}\right]\right] h_{2}, 1_{R_{1}}\right\rangle\right| \lesssim \operatorname{Off}_{\left(s_{1}, s_{2}\right)}^{\left(t_{1}, t_{2}\right), 1 *}|R|\left|I_{1}\right|^{\frac{1}{p_{1}}-\frac{1}{q_{1}}}\left|I_{2}\right|^{\frac{1}{p_{2}}-\frac{1}{q_{2}}} .
$$

The symmetry claim follows from the symmetry claims of Lemma 4.9 .

We now define larger off-support constants that can be used to control $\operatorname{osc}_{N}^{v_{1}, v_{2}}(b, R)$, uniformly on $N$, for general $v_{1}, v_{2}$. For $s_{1}, s_{2}, t_{1}, t_{2} \in(1, \infty)$ define

$$
\widetilde{\mathrm{Offf}}_{\left(s_{1}, s_{2}\right)}^{\left(t_{1}, t_{2}\right)}=\sup \left|\iint_{\mathbb{R}^{d} \times \mathbb{R}^{d}} B(x, y) K_{1}\left(x_{1}, y_{1}\right) K_{2}\left(x_{2}, y_{2}\right) f_{1}(y) f_{2}(x) \mathrm{d} y \mathrm{~d} x\right|,
$$

where the supremum is taken over rectangles $P_{1}=J_{1} \times J_{2}$ and $P_{2}=L_{1} \times L_{2}$ with

$$
\ell\left(J_{i}\right)=\ell\left(L_{i}\right) \quad \text { and } \quad \operatorname{dist}\left(J_{i}, L_{i}\right) \sim \ell\left(J_{i}\right)
$$

and over functions $f_{j} \in L^{\infty}\left(P_{j}\right)$ with

$$
\left\|f_{1}\right\|_{L_{x_{1}}^{s_{1}} L_{x_{2}}^{s_{2}}} \leq 1 \quad \text { and } \quad\left\|f_{2}\right\|_{L_{x_{1}}^{t_{1}^{\prime}} L_{x_{2}}^{t_{2}^{\prime}}} \leq 1
$$

We also define the dual off-support constants $\widetilde{\mathrm{Off}}_{\left(s_{1}, s_{2}\right)}^{\left(t_{1}, t_{2}\right), 1 *}, \widetilde{\mathrm{Off}}_{\left(s_{1}, s_{2}\right)}^{\left(t_{1}, t_{2}\right), 2 *}$ and $\widetilde{\mathrm{Off}}_{\left(s_{1}, s_{2}\right)}^{\left(t_{1}, t_{2}\right) *}$ in the natural way. Notice that $\operatorname{Off}_{\left(s_{1}, s_{2}\right)}^{\left(t_{1}, t_{2}\right)} \leq \widetilde{\mathrm{Off}}_{\left(s_{1}, s_{2}\right)}^{\left(t_{1}, t_{2}\right)}$, and if $T_{i}$ is a non-degenerate CZO with 
kernel $K_{i}$, then $\widetilde{\mathrm{Off}}_{\left(s_{1}, s_{2}\right)}^{\left(t_{1}, t_{2}\right)} \leq\left\|\left[T_{1},\left[b, T_{2}\right]\right]\right\|_{L_{x_{1}}^{s_{1}} L_{x_{2}}^{s_{2}} \rightarrow L_{x_{1}}^{t_{1}} L_{x_{2}}^{t_{2}}}$ whenever the commutator exists as a bounded linear mapping. Notice also that $\widetilde{\mathrm{Off}}_{\left(s_{1}, s_{2}\right)}^{\left(t_{1}, t_{2}\right), *}=\widetilde{\mathrm{Off}}_{\left(t_{1}^{\prime}, t_{2}^{\prime}\right)}^{\left(s_{1}^{\prime}, s^{\prime}\right)}$.

4.19. Theorem. Let $K_{i}$ be a non-degenerate kernel on $\mathbb{R}^{d_{i}}, i=1,2$, and let $b \in L_{\text {loc }}^{1}$. Let $p_{1}, p_{2}, q_{1}, q_{2} \in(1, \infty)$. Then we have for all rectangles $R=I_{1} \times I_{2}$ that

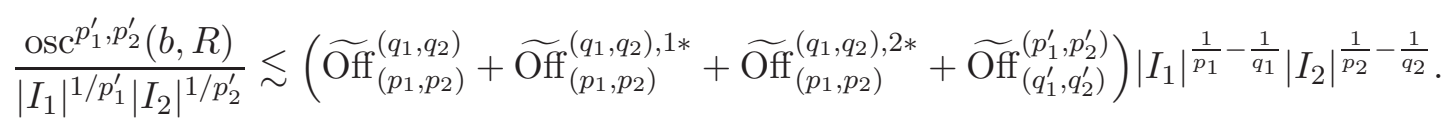

If one of the kernels $K_{i}$ is symmetrically non-degenerate, we can drop the terms $\widetilde{\mathrm{Off}}_{\left(p_{1}, p_{2}\right)}^{\left(q_{1}, q_{2}\right), 1 *}$ and $\widetilde{\mathrm{Off}}_{\left(p_{1}, p_{2}\right)}^{\left(q_{1}, q_{2}\right), 2 *}$, and if both $K_{1}$ and $K_{2}$ are symmetrically non-degenerate, then

$$
\frac{\operatorname{Osc}^{p_{1}^{\prime}, p_{2}^{\prime}}(b, R)}{\left|I_{1}\right|^{1 / p_{1}^{\prime}}\left|I_{2}\right|^{1 / p_{2}^{\prime}}} \lesssim{\widetilde{\mathrm{Off}^{\prime}}}_{\left(p_{1}, p_{2}\right)}^{\left(q_{1}, q_{2}\right)}\left|I_{1}\right|^{\frac{1}{p_{1}}-\frac{1}{q_{1}}}\left|I_{2}\right|^{\frac{1}{p_{2}}-\frac{1}{q_{2}}}
$$

Proof. Follows directly from Lemma 4.9 similarly to Theorem 4.18 .

\section{NECESSARY CONDITIONS FOR COMMUTATOR BOUNDEDNESS}

For many of the commutator lower bounds (necessary conditions) we have already done the main work; oscillatory chararacterizations of function spaces from Section 3 combined with the control of oscillations by off-support constants, Theorem 4.18 and Theorem 4.19, will yield the results. However, when there is an $\dot{L}^{r}$ norm involved, we will still have to use Lemma 4.9 directly, and we will also need to introduce yet another off-support constant.

Many of our necessary conditions are optimal; we will later be able to prove the sufficiency of the obtained necessary condition, see Theorem 1.3. A reason, but not the only reason, a lower bound might not be optimal is that the techniques used to obtain the lower bounds cannot distinguish between symmetric cases like

$$
p_{1}<q_{1} \text { and } p_{2}=q_{2}
$$

and

$$
p_{1}=q_{1} \text { and } p_{2}<q_{2},
$$

and yield symmetric lower bounds: in the first case we can bound $\|b\|_{\dot{C}_{x_{1}}^{0, \beta_{1}}\left(\mathrm{BMO}_{x_{2}}\right)}$ and in the second case $\|b\|_{\dot{C}_{x_{2}}^{0, \beta_{2}}\left(\mathrm{BMO}_{x_{1}}\right)}$ by $\left\|\left[T_{1},\left[b, T_{2}\right]\right]\right\|_{L_{x_{1}}^{p_{1}} L_{x_{2}}^{p_{2}} \rightarrow L_{x_{1}}^{q_{1}} L_{x_{2}}^{q_{2}}}$. Somewhat strikingly, both of these conditions happen to be optimal. However, e.g. in the upper right corner of the table of Theorem 1.3, where $p_{1}>q_{1}$ and $p_{2}<q_{2}$, the conditions do not match, while in the symmetric case $p_{1}<q_{1}$ and $p_{2}>q_{2}$ (the lower left corner) they do match.

To have clean statements and to avoid repetition, we will state all of the commutator lower bounds for symmetrically non-degenerate kernels $K_{i}$. From the results of Section 4 it is clear that this is not necessary - the general case just requires various dual off-support constants, and, as we saw, often it is enough that only one of the kernels is symmetrically non-degenerate to get clean statements.

Sometimes we only obtain a necessary condition in terms of an oscillatory condition that we cannot relate to a function space norm. These are recorded in this section but are not visible in the table of Theorem 1.3 (the three cases on the lower right corner). 
In what follows we always have $p_{1}, p_{2}, q_{1}, q_{2} \in(1, \infty)$, and

$$
\beta_{i}:=d_{i}\left(\frac{1}{p_{i}}-\frac{1}{q_{i}}\right), \quad \text { if } \quad p_{i}<q_{i} ; \quad \frac{1}{r_{i}}:=\frac{1}{q_{i}}-\frac{1}{p_{i}}, \quad \text { if } \quad p_{i}>q_{i} .
$$

Moreover, we also let $b \in L_{\text {loc }}^{1}$ and $K_{i}$ be a symmetrically non-degenerate kernel on $\mathbb{R}^{d_{i}}$, $i=1,2$. This data is not repeated in the statements of the results.

\section{A. The condition $\dot{C}^{0, \beta_{1}}\left(\dot{C}^{0, \beta_{2}}\right)$.}

5.1. Proposition. If $p_{1}<q_{1}$ and $p_{2}<q_{2}$, then

$$
\|b\|_{\dot{C}^{0, \beta_{1}}\left(\dot{C}^{\left.0, \beta_{2}\right)}\right.} \lesssim \operatorname{Off}_{\left(p_{1}, p_{2}\right)}^{\left(q_{1}, q_{2}\right)} .
$$

Proof. By Proposition 3.5 we have

$$
\|b\|_{\dot{C}^{0, \beta_{1}}\left(\dot{C}^{0, \beta_{2}}\right)} \sim \sup \left\{\frac{1}{\ell\left(I_{1}\right)^{\beta_{1} \ell\left(I_{2}\right)^{\beta_{2}}}} \frac{\operatorname{osc}^{1,1}\left(b, I_{1} \times I_{2}\right)}{\left|I_{1}\right|\left|I_{2}\right|}: I_{i} \subset \mathbb{R}^{d_{i}} \text { is a cube }\right\},
$$

where, by Theorem 4.18 ,

$$
\frac{1}{\ell\left(I_{1}\right)^{\beta_{1}} \ell\left(I_{2}\right)^{\beta_{2}}} \frac{\operatorname{osc}^{1,1}\left(b, I_{1} \times I_{2}\right)}{\left|I_{1}\right|\left|I_{2}\right|} \lesssim \frac{1}{\ell\left(I_{1}\right)^{\beta_{1} \ell}\left(I_{2}\right)^{\beta_{2}}} \operatorname{Off}_{\left(p_{1}, p_{2}\right)}^{\left(q_{1}, q_{2}\right)}\left|I_{1}\right|^{\frac{1}{p_{1}}-\frac{1}{q_{1}}}\left|I_{2}\right|^{\frac{1}{p_{2}}-\frac{1}{q_{2}}}=\operatorname{Off}_{\left(p_{1}, p_{2}\right)}^{\left(q_{1}, q_{2}\right)} .
$$

\section{B. The condition $\dot{C}^{0, \beta}(\mathrm{BMO})$.}

5.2. Proposition. If $p_{1}<q_{1}$ and $p_{2}=q_{2}$, then

$$
\|b\|_{\dot{C}_{x_{1}}^{0, \beta_{1}}\left(\mathrm{BMO}_{x_{2}}\right)} \lesssim \mathrm{Off}_{\left(p_{1}, p_{2}\right)}^{\left(q_{1}, p_{2}\right)} .
$$

If $p_{1}=q_{1}$ and $p_{2}<q_{2}$, then

$$
\|b\|_{\dot{C}_{x_{2}}^{0, \beta_{2}}\left(\mathrm{BMO}_{x_{1}}\right)} \lesssim \mathrm{Off}_{\left(p_{1}, p_{2}\right)}^{\left(p_{1}, q_{2}\right)} .
$$

Proof. Let $p_{1}<q_{1}$ and $p_{2}=q_{2}$. By Proposition 3.6 we have

$$
\|b\|_{\dot{C}_{x_{1}}^{0, \beta_{1}}\left(\mathrm{BMO}_{x_{2}}\right)} \sim \sup \left\{\frac{1}{\ell\left(I_{1}\right)^{\beta_{1}}} \frac{\operatorname{osc}^{1,1}\left(b, I_{1} \times I_{2}\right)}{\left|I_{1}\right|\left|I_{2}\right|}: I_{i} \subset \mathbb{R}^{d_{i}} \text { is a cube }\right\},
$$

where, by Theorem 4.18 ,

$$
\frac{1}{\ell\left(I_{1}\right)^{\beta_{1}}} \frac{\operatorname{osc}^{1,1}\left(b, I_{1} \times I_{2}\right)}{\left|I_{1}\right|\left|I_{2}\right|} \lesssim \frac{1}{\ell\left(I_{1}\right)^{\beta_{1}}} \operatorname{Off}_{\left(p_{1}, p_{2}\right)}^{\left(q_{1}, p_{2}\right)}\left|I_{1}\right|^{\frac{1}{p_{1}}-\frac{1}{q_{1}}}=\operatorname{Off}_{\left(p_{1}, p_{2}\right)}^{\left(q_{1}, p_{2}\right)} .
$$

The proof of the case $p_{1}=q_{1}$ and $p_{2}<q_{2}$ is symmetric.

5.C. The condition $\mathrm{BMO}_{\text {rect }}$. We record the following lower bound concerning the diagonal of Theorem 1.3, even though our real focus is on the off-diagonal cases.

5.3. Proposition. If $p_{1}=q_{1}$ and $p_{2}=q_{2}$ we have

$$
\|b\|_{\mathrm{BMO}_{\text {rect }}\left(p_{1}^{\prime}, p_{2}^{\prime}\right)} \lesssim \widetilde{\mathrm{Off}}_{\left(p_{1}, p_{2}\right)}^{\left(p_{1}, p_{2}\right)}
$$

Proof. By definition and Theorem 4.19 we have

$$
\|b\|_{\mathrm{BMO}_{\text {rect }}\left(p_{1}^{\prime}, p_{2}^{\prime}\right)}=\sup _{R=I_{1} \times I_{2}} \frac{\operatorname{osc}^{p_{1}^{\prime}, p_{2}^{\prime}}(b, R)}{\left|I_{1}\right|^{1 / p_{1}^{\prime}}\left|I_{2}\right|^{1 / p_{2}^{\prime}}} \lesssim{\widetilde{\mathrm{Off}^{(}}}_{\left(p_{1}, p_{2}\right)}^{\left(p_{1}, p_{2}\right)}
$$


5.D. Conditions related to $\dot{C}^{0, \beta}\left(\dot{L}^{r}\right)$ and $\operatorname{BMO}\left(\dot{L}^{r}\right)$. We need a new off-support constant to deal with the calculations arising from considerations involving the space $\dot{L}^{r}$. Define $B(x, y)=b\left(x_{1}, x_{2}\right)-b\left(x_{1}, y_{2}\right)-b\left(y_{1}, x_{2}\right)+b\left(y_{1}, y_{2}\right)$ and set

$$
\operatorname{Off}_{\left(p_{1}, p_{2}\right), \Sigma}^{\left(q_{1}, q_{2}\right)}=\sup \frac{\sum_{i=1}^{N}\left|\iint_{\mathbb{R}^{d} \times \mathbb{R}^{d}} B(x, y) K_{1}\left(x_{1}, y_{1}\right) K_{2}\left(x_{2}, y_{2}\right) f_{1, i}(y) f_{2, i}(x) \mathrm{d} y \mathrm{~d} x\right|}{\left\|\sum_{i=1}^{N}\right\| f_{1, i}\left\|_{L^{\infty}} 1_{P_{1, i}}\right\|_{L_{x_{1}}^{p_{1}} L_{x_{2}}^{p_{2}}}\left\|\sum_{i=1}^{N}\right\| f_{2, i}\left\|_{L^{\infty}} 1_{P_{2, i}}\right\|_{L_{x_{1}}^{q_{1}^{\prime}} L_{x_{2}}^{q_{2}^{\prime}}}},
$$

where the supremum is taken over rectangles $P_{1, i}=J_{1, i} \times J_{2, i}$ and $P_{2, i}=L_{1, i} \times L_{2, i}$ with

$$
\ell\left(J_{k, i}\right)=\ell\left(L_{k, i}\right) \quad \text { and } \quad \operatorname{dist}\left(J_{k, i}, L_{k, i}\right) \sim \ell\left(J_{k, i}\right)
$$

and over functions $f_{k, i} \in L^{\infty}\left(P_{k, i}\right), k=1,2, i=1, \ldots, N$. Using that for linear operators $U$ we have the random sign trick

$$
\sum_{i=1}^{N}\left\langle U f_{i}, g_{i}\right\rangle=\mathbb{E}\left\langle U\left(\sum_{i=1}^{N} \varepsilon_{i} f_{i}\right), \sum_{j=1}^{N} \varepsilon_{j} g_{j}\right\rangle,
$$

we see that $\operatorname{Off}_{\left(p_{1}, p_{2}\right), \Sigma}^{\left(q_{1}, q_{2}\right)} \leq\left\|\left[T_{1},\left[b, T_{2}\right]\right]\right\|_{L_{x_{1}}^{p_{1}} L_{x_{2}}^{p_{2}} \rightarrow L_{x_{1}}^{q_{1}} L_{x_{2}}^{q_{2}}}$ whenever the commutator exists as a bounded operator. Thus, this is a reasonable off-support constant.

5.4. Lemma. Suppose $p_{2}>q_{2}$. For all sparse collections $\mathscr{S}_{2}$ of cubes in $\mathbb{R}^{d_{2}}$, non-negative coefficients $\lambda_{S_{2}}$ satisfying $\sum_{S_{2} \in \mathscr{S}_{2}} \lambda_{S_{2}}^{r_{2}^{\prime}}\left|S_{2}\right| \leq 1$ and cubes $I_{1} \subset \mathbb{R}^{d_{1}}$, we have

$$
\sum_{S_{2} \in \mathscr{S}_{2}} \lambda_{S_{2}} \operatorname{Osc}^{1,1}\left(b, I_{1} \times S_{2}\right) \lesssim \operatorname{Off}_{\left(p_{1}, p_{2}\right), \Sigma}^{\left(q_{1}, q_{2}\right)}\left|I_{1}\right|^{1+\left(1 / p_{1}-1 / q_{1}\right)} .
$$

Proof. Using Lemma 4.9 we estimate

$$
\sum_{S_{2} \in \mathscr{S}_{2}} \lambda_{S_{2}} \operatorname{Osc}^{1,1}\left(b, I_{1} \times S_{2}\right) \lesssim \sum_{i=0}^{3} \sum_{S_{2} \in \mathscr{S}_{2}} \lambda_{S_{2}}\left|\left\langle\left[T_{1},\left[b, T_{2}\right]\right] h_{\left(I_{1} \times S_{2}\right)_{i}}, 1_{\left(I_{1} \times S_{2}\right)_{i}}\right\rangle\right|
$$

for some $h_{\left(I_{1} \times S_{2}\right)_{i}} \in L^{\infty}\left(\left(I_{1} \times S_{2}\right)_{i}\right)$ with $\left\|h_{\left(I_{1} \times S_{2}\right)_{i}}\right\|_{L^{\infty}} \lesssim 1$. Using the algebra

$$
\frac{r_{2}^{\prime}}{p_{2}}+\frac{r_{2}^{\prime}}{q_{2}^{\prime}}=r_{2}^{\prime}\left(\frac{1}{p_{2}}+1-\frac{1}{q_{2}}\right)=r_{2}^{\prime}\left(1-\frac{1}{r_{2}}\right)=1
$$

we write this in the form

$$
\sum_{i=0}^{3} \sum_{S_{2} \in \mathscr{S}_{2}}\left|\left\langle\left[T_{1},\left[b, T_{2}\right]\right]\left(\lambda_{S_{2}}^{r_{2}^{\prime} / p_{2}} h_{\left(I_{1} \times S_{2}\right)_{i}}\right), \lambda_{S_{2}}^{r_{2}^{\prime} / q_{2}^{\prime}} 1_{\left(I_{1} \times S_{2}\right)_{i}}\right\rangle\right| .
$$

This can directly be dominated with

$$
\operatorname{Off}_{\left(p_{1}, p_{2}\right), \Sigma}^{\left(q_{1}, q_{2}\right)} \sum_{i=0}^{3}\left\|\sum_{S_{2} \in \mathscr{S}_{2}} \lambda_{S_{2}}^{r_{2}^{\prime} / p_{2}} 1_{\left(I_{1} \times S_{2}\right)_{i}}\right\|\left\|_{L_{x_{1}}^{p_{1}} L_{x_{2}}^{p_{2}}}\right\| \sum_{S_{2} \in \mathscr{S}_{2}} \lambda_{S_{2}}^{r_{2}^{\prime} / q_{2}^{\prime}} 1_{\left(I_{1} \times S_{2}\right)_{i}} \|_{L_{x_{1}}^{q_{1}^{\prime}} L_{x_{2}}^{q_{2}^{\prime}}} .
$$

Here $1_{\left(I_{1} \times S_{2}\right)_{i}} \leq 1_{I_{1}^{*} \times S_{2}^{*}}$, where $I_{1}^{*} \supset I_{1} \cup \widetilde{I}_{1}$ and $S_{2}^{*} \supset S_{2} \cup \widetilde{S}_{2}$ are concentric dilations of $I_{1}$ and $S_{2}$ by a bounded factor. Since the collection $\left\{S_{2}^{*}: S_{2} \in \mathscr{S}_{2}\right\}$ is still sparse (with 
a different sparseness constant), it follows from (3.3) and $\sum_{S_{2} \in \mathscr{S}_{2}} \lambda_{S_{2}}^{r_{2}^{\prime}}\left|S_{2}\right| \leq 1$ that

$$
\begin{aligned}
& \left\|\sum_{S_{2} \in \mathscr{S}_{2}} \lambda_{S_{2}}^{r_{2}^{\prime} / p_{2}} 1_{\left(I_{1} \times S_{2}\right)_{i}}\right\|_{L_{x_{1}}^{p_{1}} L_{x_{2}}^{p_{2}}} \leq\left\|\sum_{S_{2} \in \mathscr{S}_{2}} \lambda_{S_{2}}^{r_{2}^{\prime} / p_{2}} 1_{I_{1}^{*} \times S_{2}^{*}}\right\|_{L_{x_{1}}^{p_{1}} L_{x_{2}}^{p_{2}}} \\
& =\left\|1_{I_{1}^{*}}\right\|_{L^{p_{1}}}\left\|\sum_{S_{2} \in \mathscr{S}_{2}} \lambda_{S_{2}}^{r_{2}^{\prime} / p_{2}} 1_{S_{2}^{*}}\right\|_{L^{p_{2}}} \lesssim\left|I_{1}\right|^{1 / p_{1}}\left(\sum_{S_{2} \in \mathscr{S}_{2}}\left(\lambda_{S_{2}}^{r_{2}^{\prime} / p_{2}}\right)^{p_{2}}\left|S_{2}\right|\right)^{1 / p_{2}} \leq\left|I_{1}\right|^{1 / p_{1}} .
\end{aligned}
$$

Similarly, using $1_{\left(I_{1} \times S_{2}\right)_{i}} \leq 1_{I_{1}^{*} \times S_{2}^{*}}$, we conclude that

$$
\left\|\sum_{S_{2} \in \mathscr{S}_{2}} \lambda_{S_{2}}^{r_{2}^{\prime} / q_{2}^{\prime}} 1_{\left(I_{1} \times S_{2}\right)_{i}^{\sim}}\right\|_{L_{x_{1}}^{q_{1}^{\prime}} L_{x_{2}}^{q_{2}^{\prime}}} \lesssim\left|I_{1}\right|^{1 / q_{1}^{\prime}}
$$

and this ends the proof.

The symmetric version reads as follows.

5.5. Lemma. Suppose $p_{1}>q_{1}$. For all sparse collections $\mathscr{S}_{1}$ of cubes in $\mathbb{R}^{d_{1}}$, non-negative coefficients $\lambda_{S_{1}}$ satisfying $\sum_{S_{1} \in \mathscr{I}_{1}} \lambda_{S_{1}}^{r_{1}^{\prime}}\left|S_{1}\right| \leq 1$ and cubes $I_{2} \subset \mathbb{R}^{d_{2}}$, we have

$$
\sum_{S_{1} \in \mathscr{P}_{1}} \lambda_{S_{1}} \operatorname{Osc}^{1,1}\left(b, S_{1} \times I_{2}\right) \lesssim \operatorname{Off}_{\left(p_{1}, p_{2}\right), \Sigma}^{\left(q_{1}, q_{2}\right)}\left|I_{2}\right|^{1+\left(1 / p_{2}-1 / q_{2}\right)}
$$

Relating the oscillatory constants to function spaces we can say the following.

5.6. Proposition. If $p_{1}<q_{1}$ and $p_{2}>q_{2}$ then

$$
\|b\|_{\dot{C}_{x_{1}}^{0, \beta_{1}}\left(\dot{L}_{x_{2}}^{r_{2}}\right)} \lesssim \mathrm{Off}_{\left(p_{1}, p_{2}\right), \Sigma}^{\left(q_{1}, q_{2}\right)}
$$

and if $p_{1}=q_{1}$ and $p_{2}>q_{2}$ then

$$
\begin{aligned}
\sup \left\{\frac{1}{\left|I_{1}\right|} \sum_{S_{2} \in \mathscr{S}_{2}} \lambda_{S_{2}} \operatorname{osc}^{1,1}\left(b, I_{1} \times S_{2}\right): I_{1} \subset \mathbb{R}^{d_{1}}, \mathscr{S}_{2}\right. \text { sparse, } & \left.\sum_{S_{2} \in \mathscr{S}_{2}}\left|S_{2}\right| \lambda_{S_{2}}^{r_{2}^{\prime}} \leq 1\right\} \\
& \lesssim \operatorname{Off}_{\left(p_{1}, p_{2}\right), \Sigma}^{\left(p_{1}, q_{2}\right)}
\end{aligned}
$$

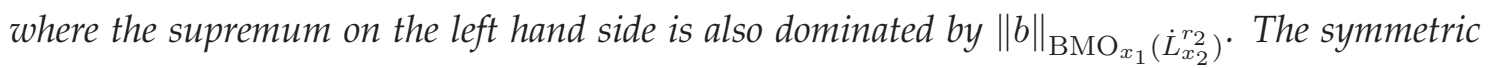
statements hold if $p_{1}>q_{1}$ and $p_{2}<q_{2}$ or $p_{1}>q_{1}$ and $p_{2}=q_{2}$.

Proof. Follows by combining the above two lemmas with Proposition 3.7 and Lemma 3.10.

\section{E. A condition related to $\dot{L}^{r_{1}}\left(\dot{L}^{r_{2}}\right)$.}

5.7. Proposition. If $p_{1}>q_{1}$ and $p_{2}>q_{2}$, then for all sparse collections $\mathscr{S}_{i}$ of cubes in $\mathbb{R}^{d_{i}}$ and non-negative coefficients $\lambda_{i, S_{i}}$ satisfying $\sum_{S_{i} \in \mathscr{S}_{i}} \lambda_{i, S_{i}}^{r_{i}^{\prime}}\left|S_{i}\right| \leq 1$ we have

$$
\sum_{S_{1} \in \mathscr{I}_{1}} \sum_{S_{2} \in \mathscr{S}_{2}} \lambda_{1, S_{1}} \lambda_{2, S_{2}} \operatorname{osc}^{1,1}\left(b, S_{1} \times S_{2}\right) \lesssim \operatorname{Off}_{\left(p_{1}, p_{2}\right), \Sigma}^{\left(q_{1}, q_{2}\right)}
$$


Proof. Using Lemma 4.9 we estimate

$$
\begin{aligned}
& \sum_{S_{1} \in \mathscr{S}_{1}} \sum_{S_{2} \in \mathscr{S}_{2}} \lambda_{1, S_{1}} \lambda_{2, S_{2}} \operatorname{osc}^{1,1}\left(b, S_{1} \times S_{2}\right) \\
& \lesssim \sum_{i=0}^{3} \sum_{S_{1} \in \mathscr{S}_{1}} \sum_{S_{2} \in \mathscr{S}_{2}} \lambda_{1, S_{1}} \lambda_{2, S_{2}}\left|\left\langle\left[T_{1},\left[b, T_{2}\right]\right] h_{\left(S_{1} \times S_{2}\right)_{i}}, 1_{\left.\left(S_{1} \times S_{2}\right)_{i}\right\rangle}\right\rangle\right| \\
&=\sum_{i=0}^{3} \sum_{S_{1} \in \mathscr{S}_{1}} \sum_{S_{2} \in \mathscr{S}_{2}}\left|\left\langle\left[T_{1},\left[b, T_{2}\right]\right]\left(\lambda_{1, S_{1}}^{r_{1}^{\prime} / p_{1}} \lambda_{2, S_{2}}^{r_{2}^{\prime} / p_{2}} h_{\left.\left(S_{1} \times S_{2}\right)_{i}\right)}\right), \lambda_{1, S_{1}}^{r_{1}^{\prime} / q_{1}^{\prime}} \lambda_{2, S_{2}}^{r_{2}^{\prime} / q_{2}^{\prime}} 1_{\left(S_{1} \times S_{2}\right)_{i}}\right\rangle\right| \\
& \leq \sum_{i=0}^{3} \operatorname{Off}_{\left(p_{1}, p_{2}\right), \Sigma}^{\left(q_{1}, q_{2}\right)}\left\|\sum_{\substack{S_{1} \in \mathscr{S}_{1} \\
S_{2} \in \mathscr{S}_{2}}} \lambda_{1, S_{1}}^{r_{1}^{\prime} / p_{1}} \lambda_{2, S_{2}}^{r_{2}^{\prime} / p_{2}} 1_{\left(S_{1} \times S_{2}\right)_{i}}\right\|_{L_{x_{1}}^{p_{1}} L_{x_{2}}^{p_{2}}} \times \\
&\left\|\sum_{\substack{S_{1} \in \mathscr{S}_{1} \\
S_{2} \in \mathscr{S}_{2}}} \lambda_{1, S_{1}}^{r_{1}^{\prime} / q_{1}^{\prime}} \lambda_{2, S_{2}}^{r_{2}^{\prime} / q_{2}^{\prime}} 1_{\left(S_{1} \times S_{2}\right)_{i}}\right\|_{L_{x_{1}}^{q_{1}^{\prime}} L_{x_{2}}^{q_{2}^{\prime}}} .
\end{aligned}
$$

Using the notation of the proof of Lemma 5.4 we have

$$
\begin{aligned}
\left\|\sum_{\substack{S_{1} \in \mathscr{S}_{1} \\
S_{2} \in \mathscr{S}_{2}}} \lambda_{1, S_{1}}^{r_{1}^{\prime} / p_{1}} \lambda_{2, S_{2}}^{r_{2}^{\prime} / p_{2}} 1_{\left(S_{1} \times S_{2}\right)_{i}}\right\|_{L_{x_{1}}^{p_{1}} L_{x_{2}}^{p_{2}}} \\
\quad \leq\left\|\sum_{\substack{S_{1} \in \mathscr{S}_{1} \\
S_{2} \in \mathscr{S}_{2}}} \lambda_{1, S_{1}}^{r_{1}^{\prime} / p_{1}} \lambda_{2, S_{2}}^{r_{2}^{\prime} / p_{2}} 1_{S_{1}^{*} \times S_{2}^{*}}\right\|_{L_{x_{1}}^{p_{1}} L_{x_{2}}^{p_{2}}} \\
\quad=\left\|\sum_{S_{1} \in \mathscr{S}_{1}} \lambda_{1, S_{1}}^{r_{1}^{\prime} / p_{1}} 1_{S_{1}^{*}}\right\|\left\|_{L_{x_{1}}^{p_{1}}}\right\| \sum_{S_{2} \in \mathscr{S}_{2}} \lambda_{2, S_{2}}^{r_{2}^{\prime} / p_{2}} 1_{S_{2}^{*}} \|_{L_{x_{2}}^{p_{2}}},
\end{aligned}
$$

where for both $i=1,2$ we have by the sparseness of the family $\left\{S_{i}^{*}: S_{i} \in \mathscr{S}_{i}\right\}$ that

$$
\left\|\sum_{S_{i} \in \mathscr{S}_{i}} \lambda_{i, S_{i}}^{r_{i}^{\prime} / p_{i}} 1_{S_{i}^{*}}\right\|_{L_{x_{i}}^{p_{i}}} \lesssim\left(\sum_{S_{i} \in \mathscr{S}_{i}}\left(\lambda_{i, S_{i}}^{r_{i}^{\prime} / p_{i}}\right)^{p_{i}}\left|S_{i}\right|\right)^{1 / p_{i}} \leq 1,
$$

and the the estimate for the $L_{x_{1}}^{q_{1}^{\prime}} L_{x_{2}}^{q_{2}^{\prime}}$ norm is completely analogous.

Of course, we would like to be able to bound $\|b\|_{\dot{L}_{x_{1}}^{r_{1}} \dot{L}_{x_{2}}^{r_{2}}}$ above, but we only know the direction (3.11).

5.8. Remark. For $p_{1}=p_{2}=: p$ and $q_{1}=q_{2}=: q$, almost the same argument gives the following slightly stronger conclusion: Suppose that $\left[T_{1},\left[b, T_{2}\right]\right]: L^{p} \rightarrow L^{q}$, where $p>q$ (this can be replaced with the off-support constants as usual). Then for $1 / r=1 / q-$ $1 / p$, any sparse collections $\mathscr{R}$ of rectangles $R=I_{1} \times I_{2}$ in $\mathbb{R}^{d_{1}} \times \mathbb{R}^{d_{2}}$ and coefficients $\sum_{R \in \mathscr{R}} \lambda_{R}^{r^{\prime}}|R| \leq 1$, we have

$$
\sum_{R \in \mathscr{R}} \lambda_{R} \operatorname{osc}^{1,1}(b, R) \lesssim\left\|\left[T_{1},\left[b, T_{2}\right]\right]\right\|_{L^{p} \rightarrow L^{q}} .
$$

The difference is that the coefficients are not of the product form, while above we had $\mathscr{R}=\mathscr{S}_{1} \times \mathscr{S}_{2}=\left\{S_{1} \times S_{2}: S_{1} \in \mathscr{S}_{1}, S_{2} \in \mathscr{S}_{2}\right\}$. The point of the generalisation is that we 
can still split the coefficients $\lambda_{R}=\lambda_{R}^{r^{\prime} / p} \lambda_{R}^{r^{\prime} / q^{\prime}}$ and estimate

$$
\left\|\sum_{R \in \mathscr{R}} \lambda_{R}^{r^{\prime} / p} 1_{R^{*}}\right\|_{L^{p}} \lesssim\left(\sum_{R \in \mathscr{R}}\left(\lambda_{R}^{r^{\prime} / p}\right)^{p}|R|\right)^{1 / p} \lesssim 1 ;
$$

in the case of mixed norms, it seems unclear how to perform this splitting of the coefficients. But even with the stronger condition, we do not know how to relate it to $b \in \dot{L}^{r}\left(\dot{L}^{r}\right)$. This problem is a variant of the question of bi-parameter sparse domination that has attracted some attention. Recently, [2] showed the failure of the natural sparse form bound for the strong maximal operator. However, our problem is more like finding a sparse bound for the identity operator!

\section{SUFFICIENT CONDITIONS FOR COMMUTATOR BOUNDEDNESS}

In this section we are given Calderón-Zygmund operators $T_{i}$ on $\mathbb{R}^{d_{i}}$ with standard kernels $K_{i}, i=1,2$. We also always assume that $b \in L_{\text {loc }}^{s}\left(\mathbb{R}^{d}\right)$ for some $s>1$. Moreover, we let $p_{1}, p_{2}, q_{1}, q_{2} \in(1, \infty)$ and use the familiar notation

$$
\beta_{i}:=d_{i}\left(\frac{1}{p_{i}}-\frac{1}{q_{i}}\right), \quad \text { if } \quad p_{i}<q_{i} ; \quad \frac{1}{r_{i}}:=\frac{1}{q_{i}}-\frac{1}{p_{i}}, \quad \text { if } \quad p_{i}>q_{i} .
$$

This fixed data is not repeated in the statements of the results.

We aim to prove

$$
\left\|\left[T_{1},\left[b, T_{2}\right]\right]\right\|_{L_{x_{1}}^{p_{1}} L_{x_{2}}^{p_{2}} \rightarrow L_{x_{1}}^{q_{1}} L_{x_{2}}^{q_{2}}} \lesssim\|b\|_{Y_{p_{i}, q_{i}}}
$$

preferably with a function space $Y_{p_{i}, q_{i}}$ that matches the corresponding necessary condition obtained in Section 5 ,

Along the way we will need several kernel representations for $\left\langle\left[T_{1},\left[b, T_{2}\right]\right] f, g\right\rangle$ even if $f$ and $g$ do not have disjoint supports. To make these rigorous we recall the notion of maximal truncations of CZOs. For $\epsilon>0$ we define for all $x_{i} \in \mathbb{R}^{d_{i}}$ and $f \in L^{p}\left(\mathbb{R}^{d_{i}}\right)$, $1 \leq p<\infty$, that

$$
T_{i, \epsilon} f\left(x_{i}\right)=\int_{\left|x_{i}-y_{i}\right|>\epsilon} K_{i}\left(x_{i}, y_{i}\right) f\left(y_{i}\right) \mathrm{d} y_{i} .
$$

We define the maximal truncation of $T_{i}$ via the formula

$$
T_{i, *} f\left(x_{i}\right)=\sup _{\epsilon>0}\left|T_{i, \epsilon} f\left(x_{i}\right)\right| .
$$

For the CZO $T_{i}$ the following Cotlar's inequality is true: for $0<r<1$ we have for all $x_{i} \in \mathbb{R}^{d_{i}}$ that

$$
T_{i, *} f\left(x_{i}\right) \lesssim r M_{r} T_{i} f\left(x_{i}\right)+M f\left(x_{i}\right) .
$$

Here $M$ is the Hardy-Littlewood maximal function on $\mathbb{R}^{d_{i}}$ and $M_{r} g=\left(M|g|^{r}\right)^{1 / s}$. The implicit constant in Cotlar's inequality depends on $\left\|T_{i}\right\|_{L^{2} \rightarrow L^{2}}$, but this is the type of CZO data that we will not track. The consequence of Cotlar's inequality is that also $T_{*}$ maps $L^{p}\left(\mathbb{R}^{d_{i}}\right)$ to $L^{p}\left(\mathbb{R}^{d_{i}}\right)$ for all $p \in(1, \infty)$. A standard fact also is that

$$
T_{i} f=T_{i, 0} f+a_{i} f
$$

where $a_{i}$ is a bounded function on $\mathbb{R}^{d_{i}}, T_{i, 0}$ is bounded on $L^{p}\left(\mathbb{R}^{d_{i}}\right)$ for all $p \in(1, \infty)$, and for some sequence $\epsilon_{j} \rightarrow 0$ we have

$$
\int T_{i, 0} f \cdot g=\lim _{j \rightarrow \infty} \int T_{i, \epsilon_{j}} f \cdot g
$$


whenever $f \in L^{p}\left(\mathbb{R}^{d_{i}}\right)$ and $g \in L^{p^{\prime}}\left(\mathbb{R}^{d_{i}}\right)$ for some $p \in(1, \infty)$.

For the kernel representations it is convenient to again denote

$$
B(x, y)=b\left(x_{1}, x_{2}\right)-b\left(x_{1}, y_{2}\right)-b\left(y_{1}, x_{2}\right)+b\left(y_{1}, y_{2}\right) .
$$

6.A. $\dot{C}^{0, \alpha}(X)$. We begin with our first kernel representation.

6.2. Proposition. If

$$
(x, y) \mapsto B(x, y) K_{1}\left(x_{1}, y_{1}\right) K_{2}\left(x_{2}, y_{2}\right) \in L_{\mathrm{loc}}^{1}\left(\mathbb{R}^{2 d}\right),
$$

then for all $f, g \in L_{c}^{\infty}\left(\mathbb{R}^{d}\right)$ we have

$$
\left\langle\left[T_{1},\left[b, T_{2}\right]\right] f, g\right\rangle=-\iint_{\mathbb{R}^{d} \times \mathbb{R}^{d}} B(x, y) K_{1}\left(x_{1}, y_{1}\right) K_{2}\left(x_{2}, y_{2}\right) f(y) g(x) \mathrm{d} y \mathrm{~d} x .
$$

Proof. We write $T_{i} h=T_{i, 0} h+a_{i} h$ as in (6.1). Notice that $\left[T_{1},\left[b, T_{2}\right]\right]=\left[T_{1,0},\left[b, T_{2,0}\right]\right]$ and that we have by definition

$$
\left[T_{1,0},\left[b, T_{2,0}\right]\right] f=T_{1,0}\left(b T_{2,0} f\right)+T_{2,0}\left(b T_{1,0} f\right)-b T_{1,0} T_{2,0} f-T_{1,0} T_{2,0}(b f) .
$$

We consider these terms separately. For all $t \in(1, s)$ we have for almost every $x_{2}$ that $x_{1} \mapsto b\left(x_{1}, x_{2}\right) T_{2,0}\left(f\left(x_{1}, \cdot\right)\right)\left(x_{2}\right) \in L^{t}\left(\mathbb{R}^{d_{1}}\right)$. Therefore, we have

$$
\left\langle T_{1,0}\left(b T_{2,0} f\right), g\right\rangle_{\mathbb{R}^{d_{1}}}=\lim _{j \rightarrow \infty}\left\langle T_{1, \varepsilon_{j}}\left(b T_{2,0} f\right), g\right\rangle_{\mathbb{R}^{d_{1}}} .
$$

Since $T_{1, *}\left(b T_{2,0} f\right) g \in L^{1}\left(\mathbb{R}^{d}\right)$ we have by dominated convergence theorem and Fubini's theorem that

$$
\left\langle T_{1,0}\left(b T_{2,0} f\right), g\right\rangle=\lim _{j \rightarrow \infty}\left\langle T_{1, \varepsilon_{j}}\left(b T_{2,0} f\right), g\right\rangle=\lim _{j \rightarrow \infty} \int_{\mathbb{R}^{d_{1}}}\left\langle T_{2,0} f, b T_{1, \varepsilon_{j}}^{*} g\right\rangle_{\mathbb{R}^{d_{2}}} .
$$

Similarly as above, we further have

$$
\left\langle T_{1,0}\left(b T_{2,0} f\right), g\right\rangle=\lim _{j \rightarrow \infty} \int_{\mathbb{R}^{d_{1}}} \lim _{k \rightarrow \infty}\left\langle T_{2, \rho_{k}} f, b T_{1, \varepsilon_{j}}^{*} g\right\rangle_{\mathbb{R}^{d_{2}}}=\lim _{j \rightarrow \infty} \lim _{k \rightarrow \infty}\left\langle T_{1, \varepsilon_{j}}\left(b T_{2, \rho_{k}} f\right), g\right\rangle .
$$

We can similarly write out all the other terms of the commutator $\left\langle\left[T_{1,0},\left[b, T_{2,0}\right]\right] f, g\right\rangle$. Thus, we have

$$
\begin{aligned}
& \left\langle\left[T_{1,0},\left[b, T_{2,0}\right]\right] f, g\right\rangle=\lim _{j \rightarrow \infty} \lim _{k \rightarrow \infty}\left\langle\left[T_{1, \varepsilon_{j}},\left[b, T_{2, \rho_{k}}\right]\right] f, g\right\rangle \\
& =-\lim _{j \rightarrow \infty} \lim _{k \rightarrow \infty} \int_{\mathbb{R}^{d}} \int_{\substack{y_{1}:\left|y_{1}-x_{1}\right|>\epsilon_{j} \\
y_{2}:\left|y_{2}-x_{2}\right|>\rho_{k}}} B(x, y) K_{1}\left(x_{1}, y_{1}\right) K_{2}\left(x_{2}, y_{2}\right) f(y) g(x) \mathrm{d} y \mathrm{~d} x .
\end{aligned}
$$

The proof is finished by a final application of the dominated convergence theorem.

We now give the upper bound that matches Proposition 5.1 .

6.3. Proposition. If $p_{1}<q_{1}$ and $p_{2}<q_{2}$, we have

$$
\left\|\left[T_{1},\left[b, T_{2}\right]\right]\right\|_{L_{x_{1}}^{p_{1}} L_{x_{2}}^{p_{2}} \rightarrow L_{x_{1}}^{q_{1}} L_{x_{2}}^{q_{2}}} \lesssim\|b\|_{\dot{C}^{0, \beta_{1}}\left(\dot{C}^{0, \beta_{2}}\right)} .
$$

Proof. By Proposition 6.2 we have for $b \in \dot{C}^{0, \beta_{1}}\left(\dot{C}^{0, \beta_{2}}\right)$ that

$$
\left|\left\langle\left[T_{1},\left[b, T_{2}\right]\right] f, g\right\rangle\right| \leq\|b\|_{\dot{C}^{0, \beta_{1}}\left(\dot{C}^{\left.0, \beta_{2}\right)}\right.}\left\langle I_{1, \beta_{1}} I_{2, \beta_{2}}|f|,|g|\right\rangle,
$$

where

$$
I_{1, \beta_{1}} f=\int_{\mathbb{R}^{d_{1}}} \frac{f\left(y_{1}\right)}{\left|x_{1}-y_{1}\right|^{d_{1}-\beta_{1}}} d y_{1}
$$


is the fractional integral hitting on $\mathbb{R}^{d_{1}}$ and $I_{2, \beta_{2}}$ is defined similarly. The proof is completed by using the well-known boundedness of the fractional integrals:

$$
I_{i, \beta_{i}}: L^{p_{i}} \rightarrow L^{q_{i}}
$$

Indeed, notice that by Minkowski's integral inequality and the positivity of the operator

$$
\left\|I_{1, \beta_{1}}|h|\right\|_{L_{x_{1}}^{q_{1}} L_{x_{2}}^{q_{2}}} \leq\left\|I_{1, \beta_{1}}\right\| h\left\|_{L_{x_{2}}^{q_{2}}}\right\|_{L_{x_{1}}^{q_{1}}} \lesssim\|\| h\left\|_{L_{x_{2}}^{q_{2}}}\right\|_{L_{x_{1}}^{p_{1}}}=\|h\|_{L_{x_{1}}^{p_{1}} L_{x_{2}}^{q_{2}}}
$$

so that no vector-valued theory is really used.

We move on to proving the remaining $\dot{C}^{0, \alpha}(X)$ type upper bounds. Again, a kernel representation is required.

6.4. Proposition. If $b \in \dot{C}_{x_{1}}^{0, \alpha}\left(\mathrm{BMO}_{x_{2}}\right)$ for some $\alpha>0$, then for all $f, g \in L_{c}^{\infty}\left(\mathbb{R}^{d}\right)$ we have

$$
\left\langle\left[T_{1},\left[b, T_{2}\right]\right] f, g\right\rangle=-\iint_{\mathbb{R}^{d_{1}} \times \mathbb{R}^{d_{1}}} K_{1}\left(x_{1}, y_{1}\right)\left\langle\left[b\left(x_{1}\right)-b\left(y_{1}\right), T_{2}\right] f\left(y_{1}\right), g\left(x_{1}\right)\right\rangle_{\mathbb{R}^{d_{2}}} .
$$

Proof. We write

$$
\begin{aligned}
\left\langle\left[T_{1},\left[b, T_{2}\right]\right] f, g\right\rangle=\left\langle\left[T_{1,0},\left[b, T_{2}\right]\right] f, g\right\rangle & =\left\langle\left[T_{1,0},\left[\widetilde{b}, T_{2}\right]\right] f, g\right\rangle \\
& =\left\langle T_{2}\left[\widetilde{b}, T_{1,0}\right] f, g\right\rangle-\left\langle\left[\widetilde{b}, T_{1,0}\right] T_{2} f, g\right\rangle,
\end{aligned}
$$

where $\widetilde{b}=b-\langle b\rangle_{V, 2}$ and $V \subset \mathbb{R}^{d_{2}}$ is a cube with $\operatorname{spt}_{\mathbb{R}^{d_{2}}} f \cup \operatorname{spt}_{\mathbb{R}^{d_{2}}} g \subset V$. We can write

$$
\begin{aligned}
\left\langle T_{2}\left[\widetilde{b}, T_{1,0}\right] f, g\right\rangle & =\left\langle T_{1,0} f, \widetilde{b} T_{2}^{*} g\right\rangle-\left\langle T_{1,0}(\widetilde{b} f), T_{2}^{*} g\right\rangle \\
& =\int_{\mathbb{R}^{d_{2}}} \lim _{j \rightarrow \infty}\left[\left\langle T_{1, \varepsilon_{j}} f, \widetilde{b} T_{2}^{*} g\right\rangle_{\mathbb{R}^{d_{1}}}-\left\langle T_{1, \varepsilon_{j}}(\widetilde{b} f), T_{2}^{*} g\right\rangle_{\mathbb{R}^{d_{1}}}\right] \\
& =\lim _{j \rightarrow \infty} \int_{\mathbb{R}^{d_{2}}}\left[\left\langle T_{1, \varepsilon_{j}} f, \widetilde{b} T_{2}^{*} g\right\rangle_{\mathbb{R}^{d_{1}}}-\left\langle T_{1, \varepsilon_{j}}(\widetilde{b} f), T_{2}^{*} g\right\rangle_{\mathbb{R}^{d_{1}}}\right],
\end{aligned}
$$

where in the last step we have used the dominated convergence theorem. Notice that we can now write the above as

$\lim _{j \rightarrow \infty} \int_{\mathbb{R}^{d_{2}}} \int_{\mathbb{R}^{d_{1}}} \int_{y_{1}:\left|x_{1}-y_{1}\right|>\varepsilon_{j}} K_{1}\left(x_{1}, y_{1}\right)\left(\widetilde{b}\left(x_{1}, y_{2}\right)-\widetilde{b}\left(y_{1}, y_{2}\right)\right) f\left(y_{1}, y_{2}\right) T_{2}^{*} g\left(x_{1}, y_{2}\right) \mathrm{d} y_{1} \mathrm{~d} x_{1} \mathrm{~d} y_{2}$.

Since $b \in \dot{C}_{x_{1}}^{0, \alpha}\left(\mathrm{BMO}_{x_{2}}\right)$, by definition we know that for all $p \in(1, \infty)$ we have

$$
y_{2} \mapsto \frac{\widetilde{b}\left(x_{1}, y_{2}\right)-\widetilde{b}\left(y_{1}, y_{2}\right)}{\left|x_{1}-y_{1}\right|^{\alpha}}=\frac{b\left(x_{1}, y_{2}\right)-b\left(y_{1}, y_{2}\right)-\left\langle b\left(x_{1}, \cdot\right)-b\left(y_{1}, \cdot\right)\right\rangle_{V}}{\left|x_{1}-y_{1}\right|^{\alpha}} \in L^{p}(V)
$$

uniformly for all $x_{1}, y_{1}$ with $x_{1} \neq y_{1}$. Using the above and the boundedness of the fractional integrals we can verify that

$$
\left(x_{1}, y_{1}, y_{2}\right) \mapsto K_{1}\left(x_{1}, y_{1}\right)\left(\widetilde{b}\left(x_{1}, y_{2}\right)-\widetilde{b}\left(y_{1}, y_{2}\right)\right) f\left(y_{1}, y_{2}\right) T_{2}^{*} g\left(x_{1}, y_{2}\right) \in L^{1}\left(\mathbb{R}^{2 d_{1}+d_{2}}\right) .
$$

Therefore, we have

$$
\begin{aligned}
& \left\langle T_{2}\left[\widetilde{b}, T_{1,0}\right] f, g\right\rangle \\
& =\int_{\mathbb{R}^{2 d_{1}}} \int_{\mathbb{R}^{d_{2}}} K_{1}\left(x_{1}, y_{1}\right) T_{2}\left(\left(\widetilde{b}\left(x_{1}, \cdot\right)-\widetilde{b}\left(y_{1}, \cdot\right)\right) f\left(y_{1}, \cdot\right)\right)\left(y_{2}\right) g\left(x_{1}, y_{2}\right) \mathrm{d} y_{2} \mathrm{~d} y_{1} \mathrm{~d} x_{1} .
\end{aligned}
$$


We complete the proof by noting that similarly $\left\langle\left[\widetilde{b}, T_{1,0}\right] T_{2} f, g\right\rangle$ equals

$$
\int_{\mathbb{R}^{2 d_{1}}} \int_{\mathbb{R}^{d_{2}}} K_{1}\left(x_{1}, y_{1}\right)\left(\widetilde{b}\left(x_{1}, y_{2}\right)-\widetilde{b}\left(y_{1}, y_{2}\right)\right) T_{2} f\left(y_{1}, y_{2}\right) g\left(x_{1}, y_{2}\right) \mathrm{d} y_{2} \mathrm{~d} y_{1} \mathrm{~d} x_{1} .
$$

We obtain the following counterpart of Proposition 5.2 .

6.5. Proposition. If $p_{1}<q_{1}$ and $p_{2}=q_{2}$, then

$$
\left\|\left[T_{1},\left[b, T_{2}\right]\right]\right\|_{L_{x_{1}}^{p_{1}} L_{x_{2}}^{p_{2}} \rightarrow L_{x_{1}}^{q_{1}} L_{x_{2}}^{p_{2}}} \lesssim\|b\|_{\dot{C}_{x_{1}}^{0, \beta_{1}}\left(\mathrm{BMO}_{x_{2}}\right)} .
$$

If $p_{1}=q_{1}$ and $p_{2}<q_{2}$, then

$$
\left\|\left[T_{1},\left[b, T_{2}\right]\right]\right\|_{L_{x_{1}}^{p_{1}} L_{x_{2}}^{p_{2}} \rightarrow L_{x_{1}}^{p_{1}} L_{x_{2}}^{q_{2}}} \lesssim\|b\|_{\dot{C}_{x_{2}}^{0, \beta_{2}}\left(\mathrm{BMO}_{x_{1}}\right)} .
$$

Proof. Let first $p_{1}<q_{1}$ and $p_{2}=q_{2}$. For $f, g \in L_{c}^{\infty}$ we have by Proposition 6.4 that

$$
\begin{aligned}
& \left|\left\langle\left[T_{1},\left[b, T_{2}\right]\right] f, g\right\rangle\right| \\
& =\left|\iint_{\mathbb{R}^{d_{1} \times \mathbb{R}^{d_{1}}}}\right| x_{1}-\left.y_{1}\right|^{\beta_{1}} K_{1}\left(x_{1}, y_{1}\right)\left\langle\left[\frac{b\left(x_{1}\right)-b\left(y_{1}\right)}{\left|x_{1}-y_{1}\right|^{\beta_{1}}}, T_{2}\right] f\left(y_{1}\right), g\left(x_{1}\right)\right\rangle_{\mathbb{R}^{d_{2}}} \mid \\
& \lesssim \iint_{\mathbb{R}^{d_{1} \times \mathbb{R}^{d_{1}}}}\left|x_{1}-y_{1}\right|^{\beta_{1}-d_{1}}\left\|\frac{b\left(x_{1}\right)-b\left(y_{1}\right)}{\left|x_{1}-y_{1}\right|^{\beta_{1}}}\right\|_{\mathrm{BMO}_{x_{2}}}\left\|f\left(y_{1}\right)\right\|_{L_{x_{2}}^{p_{2}}}\left\|g\left(x_{1}\right)\right\|_{L_{x_{2}}^{p_{2}^{\prime}}}\|\| b\left\|_{\dot{C}_{x_{1}}^{0, \beta_{1}}\left(\mathrm{BMO}_{x_{2}}\right)}\right\| f\left\|_{L_{x_{1}}^{p_{1}} L_{x_{2}}^{p_{2}}}\right\| g \|_{L_{x_{1}}^{q_{1}^{\prime}} L_{x_{2}}^{p_{2}^{\prime}},}
\end{aligned}
$$

where we have used the well-known boundedness of one parameter commutators and the fractional integrals.

Next, let $p_{1}=q_{1}$ and $p_{2}<q_{2}$. Similarly as in Proposition 6.4 for $f, g \in L_{c}^{\infty}$ we may write

$$
\left\langle\left[T_{1},\left[b, T_{2}\right]\right] f, g\right\rangle=-\iint_{\mathbb{R}^{d_{2}} \times \mathbb{R}^{d_{2}}} K_{2}\left(x_{2}, y_{2}\right)\left\langle\left[b\left(x_{2}\right)-b\left(y_{2}\right), T_{1}\right] f\left(y_{2}\right), g\left(x_{2}\right)\right\rangle_{\mathbb{R}^{d_{1}}} .
$$

By the sparse domination of commutators - see [31] - we have for all $r \in(1, \infty)$ that

$$
\begin{aligned}
\mid\left\langle\left[ b\left(x_{2}\right)-b\left(y_{2}\right)\right.\right. & \left.\left., T_{1}\right] f\left(y_{2}\right), g\left(x_{2}\right)\right\rangle_{\mathbb{R}^{d_{1}}} \mid \\
& \lesssim\left\|b\left(\cdot, x_{2}\right)-b\left(\cdot, y_{2}\right)\right\|_{\mathrm{BMO}\left(\mathbb{R}^{d_{1}}\right)} \int_{\mathbb{R}^{d_{1}}} M_{r}^{1} f\left(\cdot, y_{2}\right) M_{r}^{1} g\left(\cdot, x_{2}\right) .
\end{aligned}
$$

Choosing $1<r<\min \left\{p_{1}, p_{2}, p_{1}^{\prime}, q_{2}^{\prime}\right\}$ and using the above we have that $\left|\left\langle\left[T_{1},\left[b, T_{2}\right]\right] f, g\right\rangle\right|$ can be dominated by

$$
\begin{aligned}
\|b\|_{\dot{C}_{x_{2}}^{0, \beta_{2}}\left(\mathrm{BMO}_{x_{1}}\right)} \iint_{\mathbb{R}_{\mathbb{R}_{2} \times \mathbb{R}^{d_{2}}}}\left|K_{2}\left(x_{2}, y_{2}\right) \| x_{2}-y_{2}\right|^{\beta_{2}} \int_{\mathbb{R}_{d_{1}}} M_{r}^{1} f\left(x_{1}, y_{2}\right) M_{r}^{1} g\left(x_{1}, x_{2}\right) \\
\leq\|b\|_{\dot{C}_{x_{2}}^{0, \beta_{2}}\left(\mathrm{BMO}_{x_{1}}\right)}\|f\|_{L_{x_{1}}^{p_{1}} L_{x_{2}}^{p_{2}}}\|g\|_{L_{x_{1}}^{p_{1}^{\prime}} L_{x_{2}}^{q_{2}^{\prime}}},
\end{aligned}
$$

where we have used the boundedness of fractional integrals and mixed norm estimates of $M_{r}^{1}$. This completes the proof.

We move on to the upper bounds that are related to the Hölder space estimates of Proposition5.6. 
6.6. Proposition. If $p_{1}<q_{1}$ and $p_{2}>q_{2}$ then

$$
\left\|\left[T_{1},\left[b, T_{2}\right]\right]\right\|_{L_{x_{1}}^{p_{1}} L_{x_{2}}^{p_{2}} \rightarrow L_{x_{1}}^{q_{1}} L_{x_{2}}^{q_{2}}} \lesssim\|b\|_{\dot{C}_{x_{1}}^{0, \beta_{1}}\left(\dot{L}_{x_{2}}^{r_{2}}\right)}
$$

If $p_{1}>q_{1}$ and $p_{2}<q_{2}$ then

$$
\left\|\left[T_{1},\left[b, T_{2}\right]\right]\right\|_{L_{x_{1}}^{p_{1}} L_{x_{2}}^{p_{2}} \rightarrow L_{x_{1}}^{q_{1}} L_{x_{2}}^{q_{2}}} \lesssim\|b\|_{\dot{L}_{x_{1}}^{r_{1}}\left(\dot{C}_{x_{2}}^{0, \beta_{2}}\right)} .
$$

Proof. Let $p_{1}<q_{1}$ and $p_{2}>q_{2}$. Similarly as above for $f, g \in L_{c}^{\infty}$ we may write

$$
\left\langle\left[T_{1},\left[b, T_{2}\right]\right] f, g\right\rangle=-\iint_{\mathbb{R}^{d_{1} \times \mathbb{R}^{d_{1}}}} K_{1}\left(x_{1}, y_{1}\right)\left\langle\left[b\left(x_{1}\right)-b\left(y_{1}\right), T_{2}\right] f\left(y_{1}\right), g\left(x_{1}\right)\right\rangle_{\mathbb{R}^{d_{2}}} .
$$

This gives that

$$
\begin{aligned}
& \left|\left\langle\left[T_{1},\left[b, T_{2}\right]\right] f, g\right\rangle\right| \\
& =\left|\iint_{\mathbb{R}^{d_{1} \times \mathbb{R}^{d_{1}}}}\right| x_{1}-\left.y_{1}\right|^{\beta_{1}} K_{1}\left(x_{1}, y_{1}\right)\left\langle\left[\frac{b\left(x_{1}\right)-b\left(y_{1}\right)}{\left|x_{1}-y_{1}\right|^{\beta_{1}}}, T_{2}\right] f\left(y_{1}\right), g\left(x_{1}\right)\right\rangle_{\mathbb{R}^{d_{2}}} \mid \\
& \lesssim \iint_{\mathbb{R}^{d_{1} \times \mathbb{R}^{d_{1}}}}\left|x_{1}-y_{1}\right|^{\beta_{1}-d_{1}}\left\|\frac{b\left(x_{1}\right)-b\left(y_{1}\right)}{\left|x_{1}-y_{1}\right|^{\beta_{1}}}\right\|_{\dot{L}_{x_{2}}^{r_{2}}}\left\|f\left(y_{1}\right)\right\|_{L_{x_{2}}^{p_{2}}}\left\|g\left(x_{1}\right)\right\|_{L_{x_{2}}^{q_{2}^{\prime}}} \\
& \lesssim\|b\|_{\dot{C}_{x_{1}}^{0, \beta_{1}}\left(\dot{L}_{x_{2}}^{r_{2}}\right)}\|f\|_{L_{x_{1}}^{p_{1}} L_{x_{2}}^{p_{2}}}\|g\|_{L_{x_{1}}^{q_{1}^{\prime}} L_{x_{2}}^{q_{2}^{\prime}},}
\end{aligned}
$$

where we have used the elementary inequality (6.9) and the boundedness of fractional integrals.

Next, let $p_{1}>q_{1}$ and $p_{2}<q_{2}$. We simply estimate

$$
\begin{aligned}
\left\|\left[T_{1},\left[b, T_{2}\right]\right] f\right\|_{L_{x_{1}}^{q_{1}} L_{x_{2}}^{q_{2}}} & =\left\|\left[T_{1},\left[b-c_{2}, T_{2}\right]\right] f\right\|_{L_{x_{1}}^{q_{1}} L_{x_{2}}^{q_{2}}} \\
& \leq\left\|T_{1}\left[b-c_{2}, T_{2}\right] f\right\|_{L_{x_{1}}^{q_{1}} L_{x_{2}}^{q_{2}}}+\left\|\left[b-c_{2}, T_{2}\right] T_{1} f\right\|_{L_{x_{1}}^{q_{1}} L_{x_{2}}^{q_{2}}},
\end{aligned}
$$

use the mixed norm estimates of $T_{1}$, and the estimate

$$
\left\|\left[b-c_{2}, T_{2}\right] f\right\|_{L_{x_{1}}^{q_{1}} L_{x_{2}}^{q_{2}}} \lesssim\|\| b-c_{2}\left\|_{\dot{C}_{x_{2}}^{0, \beta_{2}}}\right\| f\left\|_{L_{x_{2}}^{p_{2}}}\right\|_{L_{x_{1}}^{q_{1}}} \leq\left\|b-c_{2}\right\|_{L_{x_{1}}^{r_{1}}\left(\dot{C}_{x_{2}}^{0, \beta_{2}}\right)}\|f\|_{L_{x_{1}}^{p_{1}} L_{x_{2}}^{p_{2}}} .
$$

To end the proof we take the infimum over suitable $c_{2}(x)=c_{2}\left(x_{2}\right)$.

6.B. BMO and $\dot{L}^{r}$. Proposition 5.6 recorded the oscillatory lower bounds related to the space $\operatorname{BMO}\left(\dot{L}^{r}\right)$. Here are the related upper bounds.

6.7. Proposition. If $p_{1}=q_{1}$ and $p_{2}>q_{2}$, then

$$
\left\|\left[T_{1},\left[b, T_{2}\right]\right]\right\|_{L_{x_{1}}^{p_{1}} L_{x_{2}}^{p_{2}} \rightarrow L_{x_{1}}^{p_{1}} L_{x_{2}}^{q_{2}}} \lesssim\|b\|_{\mathrm{BMO}_{x_{1}}\left(\dot{L}_{x_{2}}^{r_{2}}\right)} .
$$

If $p_{1}>q_{1}$ and $p_{2}=q_{2}$, then

$$
\left\|\left[T_{1},\left[b, T_{2}\right]\right]\right\|_{L_{x_{1}}^{p_{1}} L_{x_{2}}^{p_{2}} \rightarrow L_{x_{1}}^{q_{1}} L_{x_{2}}^{p_{2}}} \lesssim\|b\|_{\dot{L}_{x_{1}}^{r_{1}}\left(\mathrm{BMO}_{x_{2}}\right)} .
$$

Proof. The first estimate, where $p_{1}=q_{1}$ and $p_{2}>q_{2}$, requires some vector-valued theory of commutators. We notice that

$$
\left(\left[T_{1},\left[b, T_{2}\right]\right] f\right)^{\sharp} \lesssim \varepsilon\|b\|_{\mathrm{BMO}_{x_{1}}\left(\dot{L}_{x_{2}}^{r_{2}}\right)}\left(M_{1+\varepsilon}\left\|T_{1} f\right\|_{L_{x_{2}}^{p_{2}}}+M_{1+\varepsilon}\|f\|_{L_{x_{2}}^{p_{2}}}\right),
$$


where $M$ is the maximal function, $M_{s} g=\left(M|g|^{s}\right)^{1 / s}$ and the sharp maximal function is defined using the $L_{x_{2}}^{q_{2}}$ norm as follows

$$
g^{\sharp}\left(x_{1}\right):=\sup _{I_{1}} f_{I_{1}}\left\|g-\langle g\rangle_{I_{1}}\right\|_{L_{x_{2}}^{q_{2}}} .
$$

Here the supremum is over the cubes $I_{1} \subset \mathbb{R}^{d_{1}}$ centered at $x_{1}$. The proof of this is essentially a vector-valued version of a known pointwise bound for the sharp maximal function of $\left[b, T_{1}\right]$, but we need to keep the operator $T_{2}$ around to exploit the elementary estimate

$$
\left\|\left[b, T_{2}\right] f\right\|_{L_{x_{2}}^{q_{2}}} \lesssim\|b\|_{\dot{L}_{x_{2}}^{r_{2}}}\|f\|_{L_{x_{2}}^{p_{2}}} .
$$

We give the full details of (6.8) for the convenience of the reader. Below we repeatedly use the well-known vector-valued boundedness of $T_{1}$ and (6.9) without explicit mention.

Fix a cube $I_{1}$ containing the implicit variable. Let $f_{1}=f 1_{I_{1}^{*}}$ and $f_{2}=f-f_{1}$, where $I_{1}^{*}=5 \sqrt{d_{1}} I_{1}$. Let

We can now write

$$
c=\left\langle T_{1}\left(\left[b-a, T_{2}\right] f_{2}\right)\right\rangle_{I_{1}, 1}, \quad a=\langle b\rangle_{I_{1}^{*}, 1}
$$

$$
\left[T_{1},\left[b, T_{2}\right]\right] f=\left[T_{1},\left[b-a, T_{2}\right]\right] f=-\left[b-a, T_{2}\right] T_{1} f+T_{1}\left(\left[b-a, T_{2}\right] f_{1}\right)+T_{1}\left(\left[b-a, T_{2}\right] f_{2}\right) .
$$

Using this we split

$$
\begin{aligned}
f_{I_{1}}\left\|\left[T_{1},\left[b, T_{2}\right]\right] f-c\right\|_{L_{x_{2}}^{q_{2}}} & \leq f_{I_{1}}\left\|\left[b-a, T_{2}\right] T_{1} f\right\|_{L_{x_{2}}^{q_{2}}}+f_{I_{1}}\left\|T_{1}\left(\left[b-a, T_{2}\right] f_{1}\right)\right\|_{L_{x_{2}}^{q_{2}}} \\
& +f_{I_{1}}\left\|T_{1}\left(\left[b-a, T_{2}\right] f_{2}\right)-c\right\|_{L_{x_{2}}^{q_{2}}} \\
& =: L_{1}+L_{2}+L_{3} .
\end{aligned}
$$

We have

$$
L_{1} \lesssim\left(f_{I_{1}^{*}}\|b-a\|_{\dot{L}_{x_{2}}^{r_{2}}}^{(1+\varepsilon)^{\prime}}\right)^{\frac{1}{(1+\varepsilon)^{\prime}}}\left(f_{I_{1}}\left\|T_{1} f\right\|_{L_{x_{2}}^{p_{2}}}^{1+\varepsilon}\right)^{\frac{1}{1+\varepsilon}} \lesssim\|b\|_{\mathrm{BMO}_{x_{1}}\left(\dot{L}_{x_{2}}^{r_{2}}\right)} M_{1+\varepsilon}\left(\left\|T_{1} f\right\|_{L_{x_{2}}^{p_{2}}}\right),
$$

where in the last inequality we have used John-Nirenberg inequality of $\mathrm{BMO}_{x_{1}}\left(\dot{L}_{x_{2}}^{r_{2}}\right)$, which has the same proof as the usual vector-valued John-Nirenberg inequality (the dot in $\dot{L}_{x_{2}}^{r_{2}}$ does not matter).

We move to $L_{2}$. This time we have

$$
\begin{aligned}
L_{2} & \lesssim\left|I_{1}\right|^{-\frac{1}{1+\frac{\varepsilon}{2}}}\left\|\left[b-a, T_{2}\right] f_{1}\right\|_{L_{x_{1}}^{1+\frac{\varepsilon}{2}}\left(L_{x_{2}}^{q_{2}}\right)} \\
& \lesssim\left(f_{I_{1}^{*}}\|b-a\|_{\dot{L}_{x_{2}}^{r_{2}}}^{1+\frac{\varepsilon}{2}}\|f\|_{L_{x_{2}}^{p_{2}}}^{1+\frac{\varepsilon}{2}}\right)^{\frac{1}{1+\frac{\varepsilon}{2}}} \lesssim\|b\|_{\mathrm{BMO}_{x_{1}}\left(\dot{L}_{x_{2}}^{r_{2}}\right)} M_{1+\varepsilon}\|f\|_{L_{x_{2}}^{p_{2}}} .
\end{aligned}
$$

We used John-Nirenberg again in the last step.

It remains to estimate $L_{3}$. Since $I_{1} \cap \operatorname{spt} f_{2}=\emptyset$, using the kernel representation of $T_{1}$ and the regularity of the kernel we have

$$
L_{3} \lesssim \sum_{j=0}^{\infty} 2^{-j \alpha_{1}} f_{2^{j+1} I_{1}^{*}}\left\|\left[b-a, T_{2}\right] f\right\|_{L_{x_{2}}^{q_{2}}} \lesssim \sum_{j=0}^{\infty} 2^{-j \alpha_{1}} f_{2^{j+1} I_{1}^{*}}\|b-a\|_{\dot{L}_{x_{2}}^{r_{2}}}\|f\|_{L_{x_{2}}^{p_{2}}}
$$




$$
\begin{aligned}
& \leq \sum_{j=0}^{\infty} 2^{-j \alpha_{1}} f_{2^{j+1} I_{1}^{*}}\left\|b-\langle b\rangle_{2^{j+1} I_{1}^{*}, 1}\right\|{\dot{L}_{x_{2}}^{r_{2}}}\|f\|_{L_{x_{2}}^{p_{2}}} \\
& +\sum_{j=0}^{\infty} 2^{-j \alpha_{1}} f_{2^{j+1} I_{1}^{*}}\left\|\langle b\rangle_{I_{1}^{*}, 1}-\langle b\rangle_{2^{j+1} I_{1}^{*}, 1}\right\|_{\dot{L}_{L_{x_{2}}^{p_{2}}}^{r_{2}}\|f\|_{L_{x_{2}}^{p_{2}}} .}
\end{aligned}
$$

Using John-Nirenberg again, the first sum can be bounded by $\|b\|_{\mathrm{BMO}_{x_{1}}\left(\dot{L}_{x_{2}}^{r_{2}}\right)} M_{1+\varepsilon}\|f\|_{L_{x_{2}}^{p_{2}}}$. Moreover, since

$$
\left\|\langle b\rangle_{I_{1}^{*}, 1}-\langle b\rangle_{2^{j+1} I_{1}^{*}, 1}\right\|_{\dot{L}_{x_{2}}^{r_{2}}} \leq \sum_{i=0}^{j}\left\|\langle b\rangle_{2^{i+1} I_{1}^{*}, 1}-\langle b\rangle_{2^{i} I_{1}^{*}, 1}\right\|_{\dot{L}_{x_{2}}^{r_{2}}} \lesssim j\|b\|_{\mathrm{BMO}_{x_{1}}\left(\dot{L}_{x_{2}}^{r_{2}}\right)},
$$

the desired estimate follows for the second term as well. The inequality (6.8) follows.

Next, if $\varepsilon$ is so small that $p_{1} /(1+\varepsilon)>1$, we have by the standard Fefferman-Stein inequality that

$$
\begin{aligned}
\left\|\left[T_{1},\left[b, T_{2}\right]\right] f\right\|_{L_{x_{1}}^{p_{1}} L_{x_{2}}^{q_{2}}}^{p_{1}} & =\int_{\mathbb{R}^{d_{1}}}\left\|\left[T_{1},\left[b, T_{2}\right]\right] f\right\|_{L_{x_{2}}^{q_{2}}}^{p_{1}} \\
& \lesssim \int_{\mathbb{R}^{d_{1}}}\left[\left(\left[T_{1},\left[b, T_{2}\right]\right] f\right)^{\sharp}\right]^{p_{1}} \\
& \lesssim\|b\|_{\mathrm{BMO}_{x_{1}}\left(\dot{L}_{x_{2}}^{r_{2}}\right)}^{p_{1}}\left(\left\|M_{1+\varepsilon}\right\| T_{1} f\left\|_{L_{x_{2}}^{p_{2}}}\right\|_{L_{x_{1}}^{p_{1}}}^{p_{p_{1}}}+\left\|M_{1+\varepsilon}\right\| f\left\|_{L_{x_{2}}^{p_{2}}}\right\|_{L_{x_{1}}^{p_{1}}}^{p_{p_{1}}}\right) \\
& \lesssim\|b\|_{\mathrm{BMO}_{x_{1}}\left(\dot{L}_{x_{2}}^{r_{2}}\right)}^{p_{1}}\|f\|_{L_{x_{1}}^{p_{1}} L_{x_{2}}^{p_{2}}}^{p_{2}} .
\end{aligned}
$$

We are done with the case $p_{1}=q_{1}$ and $p_{2}>q_{2}$. See Section 7 for more about vectorvalued estimates of commutators.

The case $p_{1}>q_{1}$ and $p_{2}=q_{2}$ is the following elementary deduction. Estimate

$$
\begin{aligned}
\left\|\left[T_{1},\left[b, T_{2}\right]\right] f\right\|_{L_{x_{1}}^{q_{1}} L_{x_{2}}^{p_{2}}} & =\left\|\left[T_{1},\left[b-c_{2}, T_{2}\right]\right] f\right\|_{L_{x_{1}}^{q_{1}} L_{x_{2}}^{p_{2}}} \\
& \leq\left\|T_{1}\left[b-c_{2}, T_{2}\right] f\right\|_{L_{x_{1}}^{q_{1}} L_{x_{2}}^{p_{2}}}+\left\|\left[b-c_{2}, T_{2}\right] T_{1} f\right\|_{L_{x_{1}}^{q_{1}} L_{x_{2}}^{p_{2}}},
\end{aligned}
$$

use the mixed norm estimates of $T_{1}$ and

$$
\left\|\left[b-c_{2}, T_{2}\right] f\right\|_{L_{x_{1}}^{q_{1}} L_{x_{2}}^{p_{2}}} \lesssim\|\| b-c_{2}\left\|_{\mathrm{BMO}_{x_{2}}}\right\| f\left\|_{L_{x_{2}}^{p_{2}}}\right\|_{L_{x_{1}}^{q_{1}}} \leq\left\|b-c_{2}\right\|_{L_{x_{1}}^{r_{1}}\left(\mathrm{BMO}_{x_{2}}\right)}\|f\|_{L_{x_{1}}^{p_{1}} L_{x_{2}}^{p_{2}}} .
$$

To end the proof we take the infimum over suitable $c_{2}(x)=c_{2}\left(x_{2}\right)$.

The oscillatory lower bound related to $\dot{L}^{r_{1}} \dot{L}^{r_{2}}$ was recorded in Proposition 5.7 The upper bound is completely elementary and does not utilize any cancellation of the bicommutator.

6.10. Proposition. If $p_{1}>q_{1}$ and $p_{2}>q_{2}$, then

$$
\left\|\left[T_{1},\left[b, T_{2}\right]\right]\right\|_{L_{x_{1}}^{p_{1}} L_{x_{2}}^{p_{2}} \rightarrow L_{x_{1}}^{q_{1}} L_{x_{2}}^{q_{2}}} \lesssim\|b\|_{\dot{L}_{x_{1}}^{r_{1}}\left(\dot{L}_{x_{2}}^{r_{2}}\right)} .
$$

Proof. Simply use Hölder's inequality and the mixed-norm estimates of $T_{1}$ and $T_{2}$. 


\section{VECTOR-VALUED ESTIMATES FOR COMMUTATORS}

In the proof of Proposition 6.7 we used some vector-valued theory of commutators. Although the proof of Proposition 6.7 was completely self-contained, in this section we more systematically prove, and partly recall, such vector-valued estimates. This topic is of independent interest.

7.A. Vector-valued estimates for $[b, T]$. In the one-parameter setting $[b, T]$, where $T$ is a $\mathrm{CZO}$ in $\mathbb{R}^{d}$, the vector-valued estimates are known and quite easy, but perhaps not so well-known in the community. They are simple as there is a certain pointwise estimate for the sharp maximal function of $[b, T]$, which has a straightforward vector-valued extension. A reference is [44]. This idea was already used in the proof of Proposition 6.7. We now recall how this can be done in a general situation.

First, a few definitions. For an extensive treatment of Banach space theory see the books [24, 25] by Hytönen, van Neerven, Veraar and Weis.

Given a Banach space $X$ with norm $|\cdot|_{X}$ we let $L^{p}\left(\mathbb{R}^{d} ; X\right), p \in(0, \infty]$, be the Bochner space of $X$-valued functions with $\int_{\mathbb{R}^{d}}|f(x)|_{X}^{p} \mathrm{~d} x<\infty$. For $f: \mathbb{R}^{d} \rightarrow X$, we also define

$$
f^{\sharp}(x):=\sup _{Q=Q(x, r), r>0} \frac{1}{|Q|} \int_{Q}\left|f-\langle f\rangle_{Q}\right|_{X},
$$

where the supremum is over the cubes $Q(x, r)$ centred at $x$. We have

$$
f^{\sharp}(x) \sim \sup _{Q=Q(x, r), r>0} \inf _{e \in X} \frac{1}{|Q|} \int_{Q}|f-e|_{X} .
$$

The UMD property is a necessary and sufficient condition for the boundedness of various one-parameter singular integrals on $L^{p}\left(\mathbb{R}^{d} ; X\right)=L^{p}(X)$, see [24, Sec. 5.2.c and the Notes to Sec. 5.2]. We essentially only need to blackbox this fact, but below is the definition.

7.1. Definition. A Banach space $X$ is said to be a UMD space, where UMD stands for unconditional martingale differences, if for all $p \in(1, \infty)$, all $X$-valued $L^{p}$-martingale difference sequences $\left(d_{j}\right)_{j=1}^{k}$ and all choices of signs $\varepsilon_{j} \in\{-1,1\}$ we have

$$
\left\|\sum_{j=1}^{k} \varepsilon_{j} d_{j}\right\|_{L^{p}(X)} \lesssim\left\|\sum_{j=1}^{k} d_{j}\right\|_{L^{p}(X)} .
$$

The $L^{p}(X)$-norm is with respect to the measure space where the martingale differences are defined.

A standard property of UMD spaces is that if (7.2) holds for one $p_{0} \in(1, \infty)$ it holds for all $p \in(1, \infty)$ [24, Theorem 4.2.7]. Moreover, if $X$ is UMD then so is the dual space $X^{*}$ [24, Prop. 4.2.17].

Suppose now that $X_{1}$ is a Banach space and $X_{2}, X_{3}$ are UMD spaces such that there exists a bilinear map $X_{1} \times X_{2} \rightarrow X_{3}$, which we denote by

$$
\left(x_{1}, x_{2}\right) \rightarrow x_{1} x_{2},
$$

so that

$$
\left|x_{1} x_{2}\right|_{X_{3}} \leq\left|x_{1}\right|_{X_{1}}\left|x_{2}\right|_{X_{2}}
$$


Let $f$ take values in $X_{2}$ and $b$ take values in $X_{1}$. For a CZO $T$ on $\mathbb{R}^{d}$ a standard estimate as in the proof of Proposition 6.7 gives that for all $\varepsilon>0$ we have

$$
([b, T] f)^{\sharp} \lesssim_{\varepsilon}\|b\|_{\operatorname{BMO}\left(X_{1}\right)}\left(M_{1+\varepsilon}\left(|T f|_{X_{2}}\right)+M_{1+\varepsilon}|f|_{X_{2}}\right),
$$

where $M$ is the maximal function and $M_{s} g=\left(M|g|^{s}\right)^{1 / s}$. The sharp maximal function is defined using the norm of $X_{3}$. To get this estimate, already the boundedness $T: L^{q}\left(X_{3}\right) \rightarrow L^{q}\left(X_{3}\right), q \in(1, \infty)$, is required - this is true as $X_{3}$ is UMD. The vectorvalued estimate

$$
\|[b, T] f\|_{L^{p}\left(X_{3}\right)} \lesssim\|b\|_{\mathrm{BMO}\left(X_{1}\right)}\|f\|_{L^{p}\left(X_{2}\right)}, \quad p \in(1, \infty),
$$

follows from (7.3) using the Fefferman-Stein inequality as in the proof of Proposition 6.7 Here the UMD property of $X_{2}$ is needed via the boundedness $T: L^{p}\left(X_{2}\right) \rightarrow L^{p}\left(X_{2}\right)$.

Notice that the proof of Proposition 6.7 was almost just this vector-valued estimate we just needed to work a bit more to get the norm $\|b\|_{\mathrm{BMO}_{x_{1}}\left(\dot{L}_{x_{2}}^{r_{2}}\right)}$ instead of $\|b\|_{\mathrm{BMO}_{x_{1}}\left(L_{x_{2}}^{r_{2}}\right)}$.

Alternatively, it is also possible to directly prove a vector-valued version of the sparse domination of commutators [31]:

$$
\begin{aligned}
|\langle[b, T] f, g\rangle| & \lesssim \sum_{Q \in \mathcal{S}}\left\langle\left|b-\langle b\rangle_{Q}\right|_{X_{1}}|f|_{X_{2}}\right\rangle_{Q}\left\langle|g|_{X_{3}^{*}}\right\rangle_{Q}|Q| \\
& +\sum_{Q \in \mathcal{S}}\left\langle|f|_{X_{2}}\right\rangle_{Q}\left\langle\left|b-\langle b\rangle_{Q}\right|_{X_{1}}|g|_{X_{3}^{*}}\right\rangle_{Q}|Q| .
\end{aligned}
$$

7.B. Vector-valued estimates for bi-commutators. We will prove the bi-parameter analogue of the vector-valued estimate (7.4). There is no equally cheap way for this as in the above one-parameter case. However, we want to show that it can still be done with different methods.

We need more definitions and tools.

Dyadic notation. Let $\mathcal{D}$ be some fixed dyadic lattice in $\mathbb{R}^{d}$. For a fixed $Q \in \mathcal{D}$ and $f \in$ $L_{\text {loc }}^{1}(X)$ we define as follows.

- If $k \in \mathbb{Z}, k \geq 0$, then $Q^{(k)}$ denotes the unique cube $S \in \mathcal{D}$ for which $Q \subset S$ and $\ell(Q)=2^{-k} \ell(S)$.

- The dyadic children of $Q$ are denoted by $\operatorname{ch}(Q)=\left\{Q^{\prime} \in \mathcal{D}:\left(Q^{\prime}\right)^{(1)}=Q\right\}$.

- The average operator is $E_{Q} f=\langle f\rangle_{Q} 1_{Q}$.

- The martingale difference $\Delta_{Q} f$ is defined by $\Delta_{Q} f=\sum_{Q^{\prime} \in \operatorname{ch}(Q)} E_{Q^{\prime}} f-E_{Q} f$.

- For $k \in \mathbb{Z}, k \geq 0$, we define the martingale difference block

$$
\Delta_{Q, k} f=\sum_{\substack{S \in \mathcal{D} \\ S^{(k)}=Q}} \Delta_{S} f .
$$

Haar functions are used to decompose martingale differences $\Delta_{Q} f$ in terms of rankone operators. For an interval $I \subset \mathbb{R}$, we denote by $I_{l}$ and $I_{r}$ the left and right halves of the interval $I$, respectively. We define $h_{I}^{0}=|I|^{-1 / 2} 1_{I}$ and $h_{I}^{1}=|I|^{-1 / 2}\left(1_{I_{l}}-1_{I_{r}}\right)$. Let now $Q=I_{1} \times \cdots \times I_{d} \in \mathcal{D}$, and define the Haar function $h_{Q}^{\eta}, \eta=\left(\eta_{1}, \ldots, \eta_{d}\right) \in\{0,1\}^{d}$, via

$$
h_{Q}^{\eta}=h_{I_{1}}^{\eta_{1}} \otimes \cdots \otimes h_{I_{d}}^{\eta_{d}} .
$$


If $\eta \neq 0$, the Haar function is cancellative: $\int h_{Q}^{\eta}=0$. We may write $\Delta_{Q} f=\sum_{\eta \neq 0}\left\langle f, h_{Q}^{\eta}\right\rangle h_{Q^{\prime}}^{\eta}$ where $\left\langle f, h_{Q}^{\eta}\right\rangle=\int f h_{Q}^{\eta}$. We exploit notation by suppressing the presence of $\eta$, and simply write $h_{Q}$ for some $h_{Q}^{\eta}, \eta \neq 0$. Similarly, we write $\Delta_{Q} f=\left\langle f, h_{Q}\right\rangle h_{Q}$.

When performing bi-parameter analysis and thinking of $\mathbb{R}^{d}$ as a product space $\mathbb{R}^{d_{1}} \times$ $\mathbb{R}^{d_{2}}$, we denote a dyadic grid in $\mathbb{R}^{d_{i}}$ by $\mathcal{D}^{i}$ and denote the related dyadic rectangles in $\mathbb{R}^{d}$ by $\mathcal{D}=\mathcal{D}^{1} \times \mathcal{D}^{2}$. If $R=I_{1} \times I_{2} \in \mathcal{D}$ we set $h_{R}=h_{I_{1}} \otimes h_{I_{2}}$. We also define $\Delta_{I_{1}}^{1} f(x):=$ $\Delta_{I_{1}}\left(f\left(\cdot, x_{2}\right)\right)\left(x_{1}\right)$ and define $\Delta_{I_{2}}^{2} f$ analogously. Then we set $\Delta_{R} f=\Delta_{I_{1}}^{1} \Delta_{I_{2}}^{2} f=\Delta_{I_{2}}^{2} \Delta_{I_{1}}^{1} f$. We record that $\Delta_{I_{1}}^{1} f=h_{I_{1}} \otimes\left\langle f, h_{I_{1}}\right\rangle_{1}, \Delta_{I_{2}}^{2} f=\left\langle f, h_{I_{2}}\right\rangle_{2} \otimes h_{I_{2}}$ and $\Delta_{R} f=\left\langle f, h_{R}\right\rangle h_{R}$. Bi-parameter martingale blocks may also be defined in the natural way

$$
\Delta_{R}^{j_{1}, j_{2}} f=\sum_{J_{1}: J_{1}^{\left(j_{1}\right)}=I_{1}} \sum_{J_{2}: J_{2}^{\left(j_{2}\right)}=I_{2}} \Delta_{J_{1} \times J_{2}} f .
$$

More about Banach spaces. In addition to UMD we will need the "property $(\alpha)^{\prime}$ ". Biparameter extensions require this - see [25. Sec. 8.3.e]. Suppose that for all $N$, all scalars $a_{i, j}$ and all $e_{i, j} \in X, 1 \leq i, j \leq N$, there holds

$$
\left(\mathbb{E} \mathbb{E}^{\prime}\left|\sum_{1 \leq i, j \leq N} \varepsilon_{i} \varepsilon_{j}^{\prime} a_{i, j} e_{i, j}\right|_{X}^{2}\right)^{1 / 2} \lesssim \max _{i, j}\left|a_{i, j}\right|\left(\mathbb{E} \mathbb{E}^{\prime}\left|\sum_{1 \leq i, j \leq N} \varepsilon_{i} \varepsilon_{j}^{\prime} e_{i, j}\right|_{X}^{2}\right)^{1 / 2} .
$$

If this holds, the Banach space $X$ is said to satisfy the property $(\alpha)$ of Pisier. Here $\left\{\varepsilon_{k}\right\}_{k}$ is a collection of independent random signs - that is, the following holds. We have $\varepsilon_{k}: \mathcal{M} \rightarrow$ $\{-1,1\}$, where $(\mathcal{M}, \rho)$ is a probability space, the collection $\left\{\varepsilon_{k}\right\}_{k}$ is independent and $\rho\left(\left\{\varepsilon_{k}=1\right\}\right)=\rho\left(\left\{\varepsilon_{k}=-1\right\}\right)=1 / 2$.

Instead of dealing with UMD spaces with property $(\alpha)$ we will be working with the formally stronger (although for concrete examples essentially the same) assumption that $X$ is a UMD function lattice. For proofs and background on function lattices see [1, 23, 36, 37]. A normed space $X$ is a Banach function lattice if the following four conditions hold. Let $(\Omega, \mathcal{A}, \mu)$ be a $\sigma$-finite measure space.

(1) Every $e \in X$ is a measurable function $e: \Omega \rightarrow \mathbb{R}$ (an equivalence class).

(2) If $e: \Omega \rightarrow \mathbb{R}$ is measurable, $u \in X$ and $|e(\omega)| \leq|u(\omega)|$ for $\mu$-a.e. $\omega \in \Omega$, then $e \in X$ and $|e|_{X} \leq|u|_{X}$.

(3) There is an element $e \in X$ so that $e>0$ (i.e. $e(\omega)>0$ for $\mu$-a.e. $\omega \in \Omega$ ).

(4) If $e_{i}, e$ are non-negative, $e_{i} \in X, e_{i} \leq e_{i+1}, e_{i}(\omega) \rightarrow e(\omega)$ for $\mu$-a.e. $\omega \in \Omega$ and $\sup _{i}\left|e_{i}\right|_{X}<\infty$, we have $e \in X$ and $\left|e_{i}\right|_{X} \rightarrow|e|_{X}=\sup _{i}\left|e_{i}\right|_{X}$.

Such a space $X$ is automatically a Banach space. For a measurable $u: \Omega \rightarrow \mathbb{R}$ with $e u \in$ $L^{1}(\mu)$ for all $e \in X$, we define

$$
\Lambda_{u}: X \rightarrow \mathbb{R}, e_{u}(e)=\int_{\Omega} e(\omega) u(\omega) \mathrm{d} \mu(\omega)
$$

In this case $\Lambda_{u} \in X^{*}$ (the dual space of $X$ ). We set $X^{\prime} \subset X^{*}$ to consist of those elements of $X^{*}$ that have the form $\Lambda_{u}$ for some $u$ like above and make the obvious identification. The Banach function lattice $X^{\prime}$ is called the Köthe dual of $X$.

We mostly work with UMD Banach function lattices, where $X^{\prime}=X^{*}$ is automatic. Indeed, UMD spaces are always reflexive, and in reflexive Banach lattices $X^{\prime}=X^{*}$ holds. 
When $X^{\prime}=X^{*}$ is not automatic, we prefer to tacitly assume it for clarity. A UMD function lattice $X$ also automatically satisfies the property $(\alpha)$ of Pisier - this explains why this assumption is not explicitly needed.

Next, we explain some maximal function estimates by Bourgain [3] and Rubio de Francia [40] that are valid in UMD function lattices (see also [16]). We also explain some standard square function estimates, see e.g. the recent paper [23].

Let $X$ be a UMD function lattice, $\mathbb{R}^{d}=\mathbb{R}^{d_{1}} \times \mathbb{R}^{d_{2}}$ and $\mathcal{D}=\mathcal{D}^{1} \times \mathcal{D}^{2}$, where $\mathcal{D}^{i}$ is a dyadic grid in $\mathbb{R}^{d_{i}}$. Given $f: \mathbb{R}^{d} \rightarrow X$ and $g: \mathbb{R}^{d_{1}} \rightarrow X$ we define the dyadic (lattice) maximal functions

$M_{\mathcal{D}^{1}} g\left(x_{1}, \omega\right):=\sup _{I \in \mathcal{D}^{1}} \frac{1_{I}\left(x_{1}\right)}{|I|} \int_{I}\left|g\left(x_{1}, \omega\right)\right| \mathrm{d} x_{1}$ and $M_{\mathcal{D}} f(x, \omega):=\sup _{R \in \mathcal{D}} \frac{1_{R}(x)}{|R|} \int_{R}|f(x, \omega)| \mathrm{d} x$.

We also set $M_{\mathcal{D}^{1}}^{1} f\left(x_{1}, x_{2}\right)=M_{\mathcal{D}^{1}}\left(f\left(\cdot, x_{2}\right)\right)\left(x_{1}\right)$. The operator $M_{\mathcal{D}^{2}}^{2}$ is defined similarly.

Similarly, define the square functions

$$
S_{\mathcal{D}} f=\left(\sum_{R \in \mathcal{D}}\left|\Delta_{R} f\right|^{2}\right)^{1 / 2}, S_{\mathcal{D}^{1}}^{1} f=\left(\sum_{I \in \mathcal{D}^{n}}\left|\Delta_{I}^{1} f\right|^{2}\right)^{1 / 2} \text { and } S_{\mathcal{D}^{2}}^{2} f=\left(\sum_{J \in \mathcal{D}^{m}}\left|\Delta_{J}^{2} f\right|^{2}\right)^{1 / 2} .
$$

Notice that $|x| \in X$ and $|x|^{2} \in X$ are defined in the natural pointwise sense, as $X$ is a function lattice. Define also

$$
S_{\mathcal{D}^{1}, M_{\mathcal{D}^{2}}} f=\left(\sum_{I \in \mathcal{D}^{1}} \frac{1_{I}}{|I|} \otimes\left[M_{\mathcal{D}^{2}}\left\langle f, h_{I}\right\rangle_{1}\right]^{2}\right)^{1 / 2}
$$

and define $S_{\mathcal{D}^{2}, M_{\mathcal{D} 1}} f$ analogously. We are ready to state the following standard estimates that are at least recorded in [23].

7.5. Lemma. Let $\mathcal{D}=\mathcal{D}^{1} \times \mathcal{D}^{2}$, where $\mathcal{D}^{i}$ is a dyadic grid in $\mathbb{R}^{d_{i}}$, and let $X$ be a UMD function lattice. Then we have for all $p_{1}, p_{2} \in(1, \infty)$ that

$$
\|f\|_{L_{x_{1}}^{p_{1}} L_{x_{2}}^{p_{2}}(X)} \sim\left\|S_{\mathcal{D}} f\right\|_{L_{x_{1}}^{p_{1}} L_{x_{2}}^{p_{2}}(X)} \sim\left\|S_{\mathcal{D}^{1}}^{1} f\right\|_{L_{x_{1}}^{p_{1}} L_{x_{2}}^{p_{2}}(X)} \sim\left\|S_{\mathcal{D}^{2}}^{2} f\right\|_{L_{x_{1}}^{p_{1}} L_{x_{2}}^{p_{2}}(X)} .
$$

Moreover, for $s \in(1, \infty)$ we have the Fefferman-Stein inequality

$$
\left\|\left(\sum_{j}\left|M f_{j}\right|^{s}\right)^{1 / s}\right\|_{L_{x_{1}}^{p_{1}} L_{x_{2}}^{p_{2}}(X)} \lesssim\left\|\left(\sum_{j}\left|f_{j}\right|^{s}\right)^{1 / s}\right\|_{L_{x_{1}}^{p_{1}} L_{x_{2}}^{p_{2}}(X)} .
$$

Here $M$ can e.g. be $M_{\mathcal{D}^{1}}^{1}$ or $M_{\mathcal{D}}$. Finally, we have

$$
\left\|S_{\mathcal{D}^{1}, M_{\mathcal{D}^{2}}} f\right\|_{L_{x_{1}}^{p_{1}} L_{x_{2}}^{p_{2}}(X)}+\left\|S_{\mathcal{D}^{2}, M_{\mathcal{D}^{1}}} f\right\|_{L_{x_{1}}^{p_{1}} L_{x_{2}}^{p_{2}}(X)} \lesssim\|f\|_{L_{x_{1}}^{p_{1}} L_{x_{2}}^{p_{2}}(X)} .
$$

Product BMO and vector-valued $H^{1}-\mathrm{BMO}$ estimates. Let again $X$ be a Banach function lattice, $\mathbb{R}^{d}=\mathbb{R}^{d_{1}} \times \mathbb{R}^{d_{2}}$ and $\mathcal{D}=\mathcal{D}^{1} \times \mathcal{D}^{2}$, where $\mathcal{D}^{i}$ is a dyadic grid in $\mathbb{R}^{d_{i}}$. A locally integrable function $b: \mathbb{R}^{d} \rightarrow X$ belongs to the dyadic $X$-valued product BMO space $\operatorname{BMO}_{\mathcal{D}, \operatorname{prod}}(p, X), 0<p<\infty$, if

$$
\sup _{\Omega} \frac{1}{|\Omega|^{1 / p}}\left\|\left(\sum_{\substack{R \in \mathcal{D} \\ R \subset \Omega}}\left|\left\langle b, h_{R}\right\rangle\right|^{2} \frac{1_{R}}{|R|}\right)^{1 / 2}\right\|_{L^{p}(X)}<\infty,
$$

where the supremum is taken over all open subsets $\Omega \subset \mathbb{R}^{d}$ of finite measure. We define $\mathrm{BMO}_{\mathcal{D} \text {,prod }}(X):=\mathrm{BMO}_{\mathcal{D} \text {,prod }}(2, X)$. With this definition we will not require a Banachvalued bi-parameter John-Nirenberg inequality for which we do not have a convenient 
reference. In the scalar-valued case it is well-known that all these norms are equivalent. The product $\mathrm{BMO}$ is the supremum over all dyadic grids of the dyadic product BMO norms.

7.6. Remark. The following explanation gives one way to arrive at the definition of product BMO in our generality. If $X$ is $\mathrm{UMD}$ and $p \in(1, \infty)$ then

$$
\left(\int_{J_{i}}\left|b-\langle b\rangle_{J_{i}}\right|_{X}^{p}\right)^{1 / p}=\left\|\sum_{\substack{I_{i} \in \mathcal{D}^{i} \\ I_{i} \subset J_{i}}}\left\langle b, h_{I_{i}}\right\rangle h_{I_{i}}\right\|_{L^{p}(X)} \sim \mathbb{E}\left\|\sum_{\substack{I_{i} \in \mathcal{D}^{i} \\ I_{i} \subset J_{i}}} \varepsilon_{I_{i}}\left\langle b, h_{I_{i}}\right\rangle \frac{1_{I_{i}}}{\left|I_{i}\right|^{1 / 2}}\right\|_{L^{p}(X)} .
$$

If $X$ is a UMD function lattice we further have

$$
\mathbb{E}\left\|\sum_{\substack{I_{i} \in \mathcal{D}^{i} \\ I_{i} \subset J_{i}}} \varepsilon_{I_{i}}\left\langle b, h_{I_{i}}\right\rangle \frac{1_{I_{i}}}{\left|I_{i}\right|^{1 / 2}}\right\|_{L^{p}(X)} \sim\left\|\left(\sum_{\substack{I_{i} \in \mathcal{D}^{i} \\ I_{i} \subset J_{i}}}\left|\left\langle b, h_{I_{i}}\right\rangle\right|^{2} \frac{1_{I_{i}}}{\left|I_{i}\right|}\right)^{1 / 2}\right\|_{L^{p}(X)} .
$$

The last similarity can be seen as follows. The Kahane-Khintchine inequality (see [24, Theorem 6.2.4]) says if $X$ is a Banach space, we have for all $x_{1}, \ldots, x_{M} \in X$ and $p, q \in$ $(0, \infty)$ that

$$
\left(\mathbb{E}\left|\sum_{m=1}^{M} \varepsilon_{m} x_{m}\right|_{X}^{p}\right)^{1 / p} \sim\left(\mathbb{E}\left|\sum_{m=1}^{M} \varepsilon_{m} x_{m}\right|_{X}^{q}\right)^{1 / q}
$$

Applying this twice we see that

$$
\mathbb{E}\left\|\sum_{\substack{I_{i} \in \mathcal{D}^{i} \\ I_{i} \subset J_{i}}} \varepsilon_{I_{i}}\left\langle b, h_{I_{i}}\right\rangle \frac{1_{I_{i}}}{\left|I_{i}\right|^{1 / 2}}\right\|_{L^{p}(X)} \sim\left\|\left(\mathbb{E}\left|\sum_{\substack{I_{i} \in \mathcal{D}^{i} \\ I_{i} \subset J_{i}}} \varepsilon_{I_{i}}\left\langle b, h_{I_{i}}\right\rangle \frac{1_{I_{i}}}{\left|I_{i}\right|^{1 / 2}}\right|_{X}^{2}\right)^{1 / 2}\right\|_{L^{p}}
$$

It remains to use the equivalence

$$
\left(\mathbb{E}\left|\sum_{j} \varepsilon_{j} e_{j}\right|_{X}^{2}\right)^{1 / 2} \sim\left|\left(\sum_{j}\left|e_{j}\right|^{2}\right)^{1 / 2}\right|_{X}
$$

for all $e_{j} \in X$. This theorem of Khintchine-Maurey holds in all Banach lattices with finite cotype - in particular, in UMD function lattices. For a proof see Theorem 7.2.13 in [25].

This explains that in one-parameter the dyadic $\operatorname{BMO}(X)$ norm (if $X$ is UMD) also has the equivalent form

$$
\sup _{J_{i} \in \mathcal{D}^{i}}\left\|\left(\sum_{\substack{I_{i} \in \mathcal{D}^{i} \\ I_{i} \subset J_{i}}}\left|\left\langle b, h_{I_{i}}\right\rangle\right|^{2} \frac{1_{I_{i}}}{\left|I_{i}\right|}\right)^{1 / 2}\right\|_{L^{p}(X)} .
$$

The product BMO is simply the bi-parameter analogue of this form, where the supremum is taken over all open sets. The rectangular BMO is the analogue of this, where the supremum is over dyadic rectangles. This is because

$$
\sum_{\substack{P \in \mathcal{D} \\ P \subset R=I_{1} \times I_{2}}}\left\langle b, h_{P}\right\rangle h_{P}=\left(b-\langle b\rangle_{I_{1}, 1}-\langle b\rangle_{I_{2}, 2}+\langle b\rangle_{R}\right) 1_{R} .
$$

Let $b: \mathbb{R}^{d} \rightarrow X$ and $f: \mathbb{R}^{d} \rightarrow X^{\prime}$, where $X$ is a Banach function lattice and $X^{\prime}$ is the Köthe dual of $X$. Denote $\langle b, f\rangle=\int_{\mathbb{R}^{d}}\{b(x), f(x)\}_{X} \mathrm{~d} x$, where $\{\cdot, \cdot\}_{X}$ denotes the duality pairing between $X$ and $X^{\prime}$, in other words, we have $\left\{e, e^{\prime}\right\}_{X}=\int_{\Omega} e(\omega) e^{\prime}(\omega) \mathrm{d} \mu(\omega)$ for $e \in X$ and $e^{\prime} \in X^{\prime}$. 
The following $H^{1}$-BMO duality in the lattice case is convenient. The proof is similar to the scalar-valued case.

7.7. Lemma. Let $X$ be a Banach function lattice. Then we have

$$
|\langle b, f\rangle| \lesssim\|b\|_{\mathrm{BMO}_{\mathcal{D}, \text { prod }}(X)}\left\|S_{\mathcal{D}} f\right\|_{L^{1}\left(X^{\prime}\right)} .
$$

Proof. We may assume $\left\|S_{\mathcal{D}} f\right\|_{L^{1}\left(X^{\prime}\right)}<\infty$. Estimate

$$
|\langle b, f\rangle| \leq \sum_{R \in \mathcal{D}}\left\{\left|\left\langle b, h_{R}\right\rangle\right|,\left|\left\langle f, h_{R}\right\rangle\right|\right\}_{X} .
$$

Given $k \in \mathbb{Z}$ we define

$$
U_{k}=\left\{x:\left|S_{\mathcal{D}} f(x)\right|_{X^{\prime}}>2^{-k}\right\},
$$

and $\widehat{\mathcal{R}}_{k}=\left\{R \in \mathcal{D}:\left|R \cap U_{k}\right|>|R| / 2\right\}$. If $R \in \widehat{\mathcal{R}}_{k}$, then $R \subset \tilde{U}_{k}:=\left\{x: M_{\mathcal{D}} 1_{U_{k}}>1 / 2\right\}$, and, of course, $\left|\tilde{U}_{k}\right| \lesssim\left|U_{k}\right|$.

For all $R_{0} \in \mathcal{D}$ and $x \in R_{0}$ we have

$$
\left|S_{\mathcal{D}} f(x)\right|_{X^{\prime}} \geq \frac{\left|\left\langle f, h_{R_{0}}\right\rangle\right|_{X^{\prime}}}{\left|R_{0}\right|^{1 / 2}}
$$

If $\left|\left\langle f, h_{R_{0}}\right\rangle\right|_{X^{\prime}}>0$, then this implies $R_{0} \in \widehat{\mathcal{R}}_{k}$ for all large enough $k$. As $\left|U_{k}\right| \rightarrow 0$ when $k \rightarrow-\infty$ we also have that $R_{0} \notin \widehat{\mathcal{R}}_{k}$ for all small enough $k$. Let $\mathcal{R}_{k}=\widehat{\mathcal{R}}_{k} \backslash \widehat{\mathcal{R}}_{k-1}, k \in \mathbb{Z}$, and notice that by above

$$
\begin{aligned}
& \sum_{R \in \mathcal{D}}\left\{\left|\left\langle b, h_{R}\right\rangle\right|,\left|\left\langle f, h_{R}\right\rangle\right|\right\}_{X} \\
& \leq 2 \sum_{k \in \mathbb{Z}} \sum_{R \in \mathcal{R}_{k}}\left\langle\left|\left\langle b, h_{R}\right\rangle\right| \frac{1_{R}}{|R|^{1 / 2}},\left|\left\langle f, h_{R}\right\rangle\right| \frac{1_{R}}{|R|^{1 / 2}} 1_{\tilde{U}_{k}} 1_{U_{k-1}^{c}}\right\rangle \\
& \lesssim \sum_{k \in \mathbb{Z}}\left\|\left(\sum_{\substack{R \in \mathcal{D} \\
R \subset \tilde{U}_{k}}}\left|\left\langle b, h_{R}\right\rangle\right|^{2} \frac{1_{R}}{|R|}\right)^{1 / 2}\right\|\left\|_{L^{2}(X)}\right\|\left(\sum_{R \in \mathcal{D}}\left|\left\langle f, h_{R}\right\rangle\right|^{2} \frac{1_{R}}{|R|}\right)^{1 / 2} 1_{\tilde{U}_{k}} 1_{U_{k-1}^{c}} \|_{L^{2}\left(X^{\prime}\right)} \\
& \leq \sum_{k \in \mathbb{Z}}\|b\|_{\mathrm{BMO}_{\mathcal{D}, \text { prod }}(X)}\left|\tilde{U}_{k}\right|^{1 / 2} \times\left\|2^{-(k-1)} 1_{\tilde{U}_{k}}\right\|_{L^{2}} \\
& \lesssim \sum_{k \in \mathbb{Z}}\|b\|_{\mathrm{BMO}_{\mathcal{D}, \text { prod }}(X)} 2^{-k}\left|U_{k}\right| \sim\|b\|_{\mathrm{BMO}_{\mathcal{D}, \text { prod }}(X)}\left\|S_{\mathcal{D}} f\right\|_{L^{1}\left(X^{\prime}\right)} .
\end{aligned}
$$

Paraproducts. When we represent a singular integral using dyadic model operators [20, 21], one type of model operator that appears is a paraproduct. However, this will not be the only source of paraproducts in our arguments. In fact, the main source comes from the desire to expand products of functions $b f$ - that appear naturally in commutators using paraproducts. In this section we explain how this is done.

For $i \in\{1,2\}$ we define the one-parameter paraproducts

$$
A_{1}^{i}(b, f)=\sum_{I_{i} \in \mathcal{D}^{i}} \Delta_{I_{i}}^{i} b \Delta_{I_{i}}^{i} f, A_{2}^{i}(b, f)=\sum_{I_{i} \in \mathcal{D}^{i}} \Delta_{I_{i}}^{i} b E_{I_{i}}^{i} f \text { and } A_{3}^{i}(b, f)=\sum_{I_{i} \in \mathcal{D}^{i}} E_{I_{i}}^{i} b \Delta_{I_{i}}^{i} f \text {. }
$$


By writing $b=\sum_{I_{i}} \Delta_{I_{i}}^{i} b$ and $f=\sum_{J_{i}} \Delta_{J_{i}}^{i} f$, and collapsing sums such as $\sum_{J_{i}: I_{i} \subsetneq J_{i}} \Delta_{J_{i}}^{i} f=$ $E_{I_{i}}^{i} f$, we formally have

$$
b f=\sum_{I_{i}} \Delta_{I_{i}}^{i} b \Delta_{I_{i}}^{i} f+\sum_{I_{i} \subsetneq J_{i}} \Delta_{I_{i}}^{i} b \Delta_{J_{i}}^{i} f+\sum_{J_{i} \subsetneq I_{i}} \Delta_{I_{i}}^{i} b \Delta_{J_{i}}^{i} f=\sum_{j=1}^{3} A_{j}^{i}(b, f) .
$$

Sometimes, when we pair $b f$ with a tensor product of Haar functions, only one of the appearing Haar functions is cancellative. In this type of a situation we expand using these one-parameter paraproducts, like above, in the parameter $i \in\{1,2\}$ where the cancellative Haar function appears.

A feature of such decompositions is that the paraproduct $A_{3}^{i}(b, f)$ appears (e.g. such paraproducts do not appear in the dyadic representation of singular integrals). Indeed, there is an average on $b$, and so no BMO type philosophy on that parameter can characterize its boundedness. However, these will appear in commutator decompositions and the cancellation present in the commutator is key to handling such terms. In fact, none of the one-parameter paraproducts are bounded by themselves with the assumption $b \in \mathrm{BMO}_{\text {prod. }}$.

We continue to define bi-parameter paraproducts, which can be obtained by chaining two one-parameter paraproducts. For $j_{1}, j_{2} \in\{1,2,3\}$ define formally

$$
A_{j_{1}, j_{2}}(b, f)=A_{j_{1}}^{1} A_{j_{2}}^{2}(b, f)
$$

so that e.g.

$$
A_{1,2}(b, f)=\sum_{I_{2} \in \mathcal{D}^{2}} A_{1}^{1}\left(\Delta_{I_{2}}^{2} b, E_{I_{2}}^{2} f\right)=\sum_{R=I_{1} \times I_{2} \in \mathcal{D}} \Delta_{R} b \Delta_{I_{1}}^{1} E_{I_{2}}^{2} f .
$$

We can again expand the product $b f$ as follows

$$
b f=\sum_{j_{1}, j_{2} \in\{1,2,3\}} A_{j_{1}, j_{2}}(b, f) .
$$

Now, those bi-parameter paraproducts where $j_{1}, j_{2} \in\{1,2\}$, will be automatically bounded if $b$ is in product BMO. The rest will have to be handled by exploiting the cancellation of the commutator.

We now formulate the vector-valued setting, where we can prove the boundedness of our paraproducts. We say that $\left(X_{1}, X_{2}, X_{3}\right)$ is a compatible triple of function lattices, if each $X_{i}$ is a Banach lattice defined using the same underlying measure space $(\Omega, \mu), X_{2}$ and $X_{3}$ are UMD, and we have

$$
\left|x_{1} x_{2}\right|_{X_{3}} \leq\left|x_{1}\right|_{X_{1}}\left|x_{2}\right|_{X_{2}} .
$$

7.8. Proposition. Let $p_{1}, p_{2} \in(1, \infty)$ and $\left(X_{1}, X_{2}, X_{3}\right)$ be a compatible triple of function lattices. For $j_{1}, j_{2} \in\{1,2\}$ we have the paraproduct estimate

$$
\left\|A_{j_{1}, j_{2}}(b, f)\right\|_{L_{x_{1}}^{p_{1}} L_{x_{2}}^{p_{2}}\left(X_{3}\right)} \lesssim\|b\|_{\mathrm{BMO}_{\mathcal{D}, \text { prod }}\left(X_{1}\right)}\|f\|_{L_{x_{1}}^{p_{1}} L_{x_{2}}^{p_{2}}\left(X_{2}\right)} .
$$

Proof. We will show the estimate for $A_{1,2}(b, f)$ - the other cases are very similar. Let $g \in L_{x_{1}}^{p_{1}^{\prime}} L_{x_{2}}^{p_{2}^{\prime}}\left(X_{3}^{\prime}\right)$ and estimate

$$
\left|\left\langle A_{1,2}(b, f), g\right\rangle\right| \leq \sum_{R=I_{1} \times I_{2}}\left\{\left|\left\langle b, h_{R}\right\rangle\right|,\left|\left\langle f, h_{I_{1}} \otimes \frac{1_{I_{2}}}{\left|I_{2}\right|}\right\rangle\right|\left|\left\langle g, h_{I_{1}} h_{I_{1}} \otimes h_{I_{2}}\right\rangle\right|\right\}
$$




$$
\lesssim\|b\|_{\mathrm{BMO}_{\mathcal{D}, \text { prod }}\left(X_{1}\right)}\left\|\left(\sum_{R=I_{1} \times I_{2}}\left|\left\langle f, h_{I_{1}} \otimes \frac{1_{I_{2}}}{\left|I_{2}\right|}\right\rangle\right|^{2}\left|\left\langle g, h_{I_{1}} h_{I_{1}} \otimes h_{I_{2}}\right\rangle\right|^{2} \frac{1_{R}}{|R|}\right)^{1 / 2}\right\|_{L^{1}\left(X_{1}^{\prime}\right)} .
$$

Here we used Lemma 7.7. We can now further dominate this by

$$
\left\|S_{\mathcal{D}^{1}, M_{\mathcal{D}^{2}}} f\right\|_{L_{x_{1}}^{p_{1}} L_{x_{2}}^{p_{2}}\left(X_{2}\right)}\left\|S_{\mathcal{D}^{2}, M_{\mathcal{D}^{1}}} g\right\|_{L_{x_{1}}^{p_{1}^{\prime}} L_{x_{2}}^{p_{2}^{\prime}}\left(X_{3}^{\prime}\right)},
$$

and the proof is finished using Lemma 7.5 .

7.9. Remark. In the one-parameter case, paraproduct estimates are known to hold in all UMD spaces (i.e., without imposing any additional function lattice structure), but the proofs become slightly more demanding under such minimal assumptions. For background and a modern proof of such estimates see e.g. [26].

We are now ready to give the proof of the bi-parameter analogue of (7.4). The proof follows the modern paradigm of dyadic representation theorems of singular integrals combined with the paraproduct decompositions of commutators.

7.10. Remark. A vector-valued estimate for $[b, T]$, where $T$ is a bi-parameter $\mathrm{CZO}$ [38], could extremely likely also be proved with an adaptation of the strategy below.

7.11. Theorem. Let $p_{1}, p_{2} \in(1, \infty)$ and $\left(X_{1}, X_{2}, X_{3}\right)$ be a compatible triple of function lattices. Let $T_{i}$ be a CZO in $\mathbb{R}^{d_{i}}, i=1,2$. Then we have

$$
\left\|\left[T_{1},\left[b, T_{2}\right]\right] f\right\|_{L_{x_{1}}^{p_{1}} L_{x_{2}}^{p_{2}}\left(X_{3}\right)} \lesssim\|b\|_{\mathrm{BMO}_{\mathrm{prod}}\left(X_{1}\right)}\|f\|_{L_{x_{1}}^{p_{1}} L_{x_{2}}^{p_{2}}\left(X_{2}\right)} .
$$

Proof. The proof is a vector-valued adaptation of the recent commutator decompositions [33, 34, 35]. In particular, see [34] for a two-weight version of this bound in the scalarvalued setting. To begin the proof, we recall that it is enough to fix lattices $\mathcal{D}^{i}$ and to estimate $\left[U_{1},\left[b, U_{2}\right]\right] f$, where $U_{1}$ and $U_{2}$ are DMOs - dyadic model operators - appearing in the representation theorem [20, 21]. That is, we have that $U_{i} \in\left\{S_{i}, \pi_{i}\right\}$, where $S_{i}$ is a so-called dyadic shift in $\mathbb{R}^{d_{i}}$ and $\pi_{i}$ is a dyadic paraproduct in $\mathbb{R}^{d_{i}}$. We will recall the definitions during the proof. It is enough to obtain a polynomial dependence on the complexity of the shift.

We will only explicitly show the case $\left[S_{1},\left[b, \pi_{2}\right]\right] f$ (see [34] for the decompositions used in the other cases). The dyadic shift $S_{1}$ has the form

$$
S_{1} f=\sum_{\substack{K_{1} \in \mathcal{D}^{1} \\ I_{1}^{\left(i_{1}\right)}=J_{1}^{\left(j_{1}\right)}=K_{1}}} a_{K_{1}, I_{1}, J_{1}} h_{J_{1}} \otimes\left\langle f, h_{I_{1}}\right\rangle_{1},
$$

where $i_{1}, j_{1} \geq 0$ and $\left|a_{K_{1}, I_{1}, J_{1}}\right| \leq\left|I_{1}\right|^{1 / 2}\left|J_{1}\right|^{1 / 2}\left|K_{1}\right|^{-1}$. The paraproduct $\pi_{2}$ has the form

$$
\pi_{2} f=\sum_{K_{2} \in \mathcal{D}^{2}} a_{K_{2}}\langle f\rangle_{K_{2}, 2} \otimes h_{K_{2}}
$$

where $\left\|\left(a_{K_{2}}\right)\right\|_{\mathrm{BMO}\left(\mathcal{D}^{2}\right)}=\sup _{P_{2} \in \mathcal{D}^{2}}\left(\frac{1}{\left|P_{2}\right|} \sum_{K_{2} \subset P_{2}}\left|a_{K_{2}}\right|^{2}\right)^{1 / 2} \leq 1$. We now write out

$$
\left[S_{1},\left[b, \pi_{2}\right]\right] f=S_{1}\left(b \pi_{2} f\right)-b \pi_{2} S_{1} f-S_{1} \pi_{2}(b f)+\pi_{2}\left(b S_{1} f\right) .
$$

We identify the products

$$
b \pi_{2} f, b \pi_{2} S_{1} f, b f, b S_{1} f
$$


that we want to expand using paraproducts. Of course, it is possible to expand these in the one-parameter sense (in either parameter) or in the bi-parameter sense. What is actually useful is determined by the form of the operators $S_{1}$ and $\pi_{2}$, and looking at where the cancellative Haar functions appear. This leads us to e.g. expand

$$
\begin{aligned}
b \pi_{2} f & =\sum_{k_{1}, k_{2} \in\{1,2,3\}} A_{k_{1}, k_{2}}\left(b, \pi_{2} f\right) \\
& =\sum_{k_{1}, k_{2} \in\{1,2\}} A_{k_{1}, k_{2}}\left(b, \pi_{2} f\right)+\sum_{\substack{\left(k_{1}, k_{2}\right) \neq(3,3) \\
3 \in\left\{k_{1}, k_{2}\right\}}} A_{k_{1}, k_{2}}\left(b, \pi_{2} f\right)+A_{3,3}\left(b, \pi_{2} f\right),
\end{aligned}
$$

where the idea of the latter grouping is that in the case $k_{1}, k_{2} \in\{1,2\}$ we can directly use Proposition 7.8, while the remaining terms cannot be handled alone. They need to be grouped with terms coming from the other product expansions, which are as follows:

$$
\begin{gathered}
b \pi_{2} S_{1} f=\sum_{k_{1}, k_{2} \in\{1,2\}} A_{k_{1}, k_{2}}\left(b, \pi_{2} S_{1} f\right)+\sum_{\substack{\left.k_{1}, k_{2}\right) \neq(3,3) \\
3 \in\left\{k_{1}, k_{2}\right\}}} A_{k_{1}, k_{2}}\left(b, \pi_{2} S_{1} f\right)+A_{3,3}\left(b, \pi_{2} S_{1} f\right), \\
b f=\sum_{k_{1}=1}^{2} A_{k_{1}}^{1}(b, f)+A_{3}^{1}(b, f)
\end{gathered}
$$

and

$$
b S_{1} f=\sum_{k_{1}=1}^{2} A_{k_{1}}^{1}\left(b, S_{1} f\right)+A_{3}^{1}\left(b, S_{1} f\right) .
$$

None of the one-parameter paraproducts are bounded as they are. However, when $k_{1} \neq$ 3 , they will be paired with one other term coming from the other product expansions. In this sense, they are similar to the terms of the form $A_{k_{1}, k_{2}}(b, F),\left(k_{1}, k_{2}\right) \neq(3,3), 3 \in$ $\left\{k_{1}, k_{2}\right\}$. Finally, all the four terms $A_{3,3}\left(b, \pi_{2} f\right), A_{3,3}\left(b, \pi_{2} S_{1} f\right), A_{3}^{1}(b, f)$ and $A_{3}^{1}\left(b, S_{1} f\right)$ will be grouped together.

The above explanation leads us to the long expansion

$$
\begin{aligned}
{\left[S_{1},\left[b, \pi_{2}\right]\right] f=} & \sum_{k_{1}, k_{2} \in\{1,2\}} S_{1}\left(A_{k_{1}, k_{2}}\left(b, \pi_{2} f\right)\right)-\sum_{k_{1}, k_{2} \in\{1,2\}} A_{k_{1}, k_{2}}\left(b, \pi_{2} S_{1} f\right) \\
+ & \left\{\sum_{\substack{\left(k_{1}, k_{2}\right) \neq(3,3) \\
3 \in\left\{k_{1}, k_{2}\right\}}} S_{1}\left(A_{k_{1}, k_{2}}\left(b, \pi_{2} f\right)\right)+\sum_{k_{1}=1}^{2} \pi_{2}\left(A_{k_{1}}^{1}\left(b, S_{1} f\right)\right)\right. \\
& \left.\quad-\sum_{\substack{\left(k_{1}, k_{2}\right) \neq(3,3) \\
3 \in\left\{k_{1}, k_{2}\right\}}} A_{k_{1}, k_{2}}\left(b, \pi_{2} S_{1} f\right)-\sum_{k_{1}=1}^{2} S_{1} \pi_{2}\left(A_{k_{1}}^{1}(b, f)\right)\right\} \\
+ & \left\{\left[S_{1}\left(A_{3,3}\left(b, \pi_{2} f\right)\right)-S_{1} \pi_{2}\left(A_{3}^{1}(b, f)\right)\right]+\left[\pi_{2}\left(A_{3}^{1}\left(b, S_{1} f\right)\right)-A_{3,3}\left(b, \pi_{2} S_{1} f\right)\right]\right\} .
\end{aligned}
$$

Using Proposition 7.8 and the fact (see [26]) that $S_{1}$ and $\pi_{2}$ are bounded in $L_{x_{1}}^{s_{1}} L_{x_{2}}^{s_{2}}(X)$ for all $s_{1}, s_{2} \in(1, \infty)$ and for all UMD function lattices $X$, we have that the first two terms in the expansion above automatically satisfy the desired estimate. The big group in the middle consists of the various terms, where exactly one of the paraproduct indices is 3 or we have a one-parameter paraproduct with an indice $k_{1} \neq 3$. They will not all be handled 
together, but rather by always pairing two suitable ones together (notice that there are 6 terms with a plus sign and 6 terms with a minus sign). It is not straightforward to explain which terms should be paired together - it can be seen by analysing their forms. We get to them later.

We start our work with the last term, which is the combination of the four terms, where all the paraproduct indices are 3 . First, we have by the definition of $A_{3,3}$ and $\pi_{2}$ that

$$
\begin{aligned}
A_{3,3}\left(b, \pi_{2} f\right) & =\sum_{L_{1}, L_{2}}\langle b\rangle_{L_{1} \times L_{2}}\left\langle\pi_{2} f, h_{L_{1}} \otimes h_{L_{2}}\right\rangle h_{L_{1}} \otimes h_{L_{2}} \\
& =\sum_{L_{1}, L_{2}}\langle b\rangle_{L_{1} \times L_{2}}\left\langle\sum_{K_{2}} a_{K_{2}}\langle f\rangle_{K_{2}, 2} \otimes h_{K_{2}}, h_{L_{1}} \otimes h_{L_{2}}\right\rangle h_{L_{1}} \otimes h_{L_{2}} \\
& =\sum_{L_{1}, K_{2}} a_{K_{2}}\langle b\rangle_{L_{1} \times K_{2}}\left\langle f, h_{L_{1}} \otimes \frac{1_{K_{2}}}{\left|K_{2}\right|}\right\rangle h_{L_{1}} \otimes h_{K_{2}} .
\end{aligned}
$$

Now, using the definition of $S_{1}$ we calculate

$$
\begin{aligned}
S_{1}\left(A_{3,3}\left(b, \pi_{2} f\right)\right)= & \sum_{\substack{K_{1} \\
I_{1}^{\left(i_{1}\right)}=J_{1}^{\left(j_{1}\right)}=K_{1}}} a_{K_{1}, I_{1}, J_{1}} h_{J_{1}} \otimes\left\langle A_{3,3}\left(b, \pi_{2} f\right), h_{I_{1}}\right\rangle_{1} \\
= & \sum_{\substack{K_{1} \\
I_{1}^{\left(i_{1}\right)}=J_{1}^{\left(j_{1}\right)}=K_{1}}} a_{K_{1}, I_{1}, J_{1}} h_{J_{1}} \otimes\left\langle\sum_{L_{1}, K_{2}} a_{K_{2}}\langle b\rangle_{L_{1} \times K_{2}}\left\langle f, h_{L_{1}} \otimes \frac{1_{K_{2}}}{\left|K_{2}\right|}\right\rangle h_{L_{1}} \otimes h_{K_{2}}, h_{I_{1}}\right\rangle_{1} \\
= & \sum_{K_{2}} a_{K_{2}} \sum_{\substack{K_{1} \\
I_{1}^{\left(i_{1}\right)}=J_{1}^{\left(j_{1}\right)}=K_{1}}} a_{K_{1}, I_{1}, J_{1}}\langle b\rangle_{I_{1} \times K_{2}}\left\langle f, h_{I_{1}} \otimes \frac{1_{K_{2}}}{\left|K_{2}\right|}\right\rangle h_{J_{1}} \otimes h_{K_{2}} .
\end{aligned}
$$

The next task is to perform a completely analogous calculation to get that

$$
S_{1} \pi_{2}\left(A_{3}^{1}(b, f)\right)=\sum_{K_{2}} a_{K_{2}} \sum_{\substack{K_{1} \\ I_{1}^{\left(i_{1}\right)}=J_{1}^{\left(j_{1}\right)}=K_{1}}} a_{K_{1}, I_{1}, J_{1}}\left\langle\langle b\rangle_{I_{1}, 1}\left\langle f, h_{I_{1}}\right\rangle_{1}\right\rangle_{K_{2}} h_{J_{1}} \otimes h_{K_{2}} .
$$

We can now write

$$
\begin{aligned}
& S_{1}\left(A_{3,3}\left(b, \pi_{2} f\right)\right)-S_{1} \pi_{2}\left(A_{3}^{1}(b, f)\right) \\
& \quad=\sum_{K_{2}} a_{K_{2}} \sum_{\substack{K_{1} \\
I_{1}^{\left(i_{1}\right)}=J_{1}^{\left(j_{1}\right)}=K_{1}}} a_{K_{1}, I_{1}, J_{1}}\left\langle\left[\langle b\rangle_{I_{1} \times K_{2}}-\langle b\rangle_{I_{1}, 1}\right]\left\langle f, h_{I_{1}}\right\rangle_{1}\right\rangle_{K_{2}} h_{J_{1}} \otimes h_{K_{2}} .
\end{aligned}
$$

Here the term $\left\langle\left[\langle b\rangle_{I_{1} \times K_{2}}-\langle b\rangle_{I_{1}, 1}\right]\left\langle f, h_{I_{1}}\right\rangle_{1}\right\rangle_{K_{2}}$ appears, which we further expand by applying the martingale difference expansion $g 1_{K_{2}}=\sum_{I_{2} \subset K_{2}}\left\langle g, h_{I_{2}}\right\rangle h_{I_{2}}+\langle g\rangle_{K_{2}} 1_{K_{2}}$ to the function $\langle b\rangle_{I_{1}, 1}$. This gives that

$$
\begin{aligned}
\left\langle\left[\langle b\rangle_{I_{1} \times K_{2}}-\langle b\rangle_{I_{1}, 1}\right]\left\langle f, h_{I_{1}}\right\rangle_{1}\right\rangle_{K_{2}} & =-\left\langle\left[\sum_{I_{2} \subset K_{2}}\left\langle\left\langle b, h_{I_{2}}\right\rangle_{2}\right\rangle_{I_{1}} h_{I_{2}}\right]\left\langle f, h_{I_{1}}\right\rangle_{1}\right\rangle_{K_{2}} \\
& =-\frac{1}{\left|K_{2}\right|} \sum_{I_{2} \subset K_{2}}\left\langle\left\langle b, h_{I_{2}}\right\rangle_{2}\right\rangle_{I_{1}}\left\langle f, h_{I_{1}} \otimes h_{I_{2}}\right\rangle .
\end{aligned}
$$


Plugging this into (7.12) gives the final formula

$$
\begin{aligned}
& S_{1}\left(A_{3,3}\left(b, \pi_{2} f\right)\right)-S_{1} \pi_{2}\left(A_{3}^{1}(b, f)\right) \\
& \quad=-\sum_{K_{2}} \frac{a_{K_{2}}}{\left|K_{2}\right|} \sum_{\substack{K_{1} \\
I_{1}^{\left(i_{1}\right)}=J_{1}^{\left(j_{1}\right)}=K_{1}}} \sum_{I_{2} \subset K_{2}} a_{K_{1}, I_{1}, J_{1}}\left\langle\left\langle b, h_{I_{2}}\right\rangle_{2}\right\rangle_{I_{1}}\left\langle f, h_{I_{1}} \otimes h_{I_{2}}\right\rangle h_{J_{1}} \otimes h_{K_{2}} .
\end{aligned}
$$

This is clear progress as there is now one cancellative Haar function paired with $b$. On the other hand, this is simply not enough, since we need two cancellative Haar functions to exploit the product BMO assumption. However, with very similar calculation as above we see that

$$
\begin{aligned}
& \pi_{2}\left(A_{3}^{1}\left(b, S_{1} f\right)\right)-A_{3,3}\left(b, \pi_{2} S_{1} f\right) \\
& \quad=\sum_{K_{2}} \frac{a_{K_{2}}}{\left|K_{2}\right|} \sum_{\substack{K_{1} \\
I_{1}^{\left(i_{1}\right)}=J_{1}^{\left(j_{1}\right)}=K_{1}}} \sum_{I_{2} \subset K_{2}} a_{K_{1}, I_{1}, J_{1}}\left\langle\left\langle b, h_{I_{2}}\right\rangle_{2}\right\rangle_{J_{1}}\left\langle f, h_{I_{1}} \otimes h_{I_{2}}\right\rangle h_{J_{1}} \otimes h_{K_{2}} .
\end{aligned}
$$

The only difference is that we have a plus sign in front and that $\left\langle\left\langle b, h_{I_{2}}\right\rangle_{2}\right\rangle_{I_{1}}$ is replaced by $\left\langle\left\langle b, h_{I_{2}}\right\rangle_{2}\right\rangle_{J_{1}}$. We have arrived at the combined formula

$$
\begin{array}{r}
{\left[S_{1}\left(A_{3,3}\left(b, \pi_{2} f\right)\right)-S_{1} \pi_{2}\left(A_{3}^{1}(b, f)\right)\right]+\left[\pi_{2}\left(A_{3}^{1}\left(b, S_{1} f\right)\right)-A_{3,3}\left(b, \pi_{2} S_{1} f\right)\right]} \\
=\sum_{K_{2}} \frac{a_{K_{2}}}{\left|K_{2}\right|} \sum_{\substack{K_{1} \\
I_{1}^{\left(i_{1}\right)}=J_{1}^{\left(j_{1}\right)}=K_{1}}} \sum_{I_{2} \subset K_{2}} a_{K_{1}, I_{1}, J_{1}}\left[\left\langle\left\langle b, h_{I_{2}}\right\rangle_{2}\right\rangle_{J_{1}}-\left\langle\left\langle b, h_{I_{2}}\right\rangle_{2}\right\rangle_{I_{1}}\right] \\
\times\left\langle f, h_{I_{1}} \otimes h_{I_{2}}\right\rangle h_{J_{1}} \otimes h_{K_{2}} .
\end{array}
$$

Next, we add and subtract $\left\langle\left\langle b, h_{I_{2}}\right\rangle_{2}\right\rangle_{K_{1}}$ and e.g. write

$$
\left\langle\left\langle b, h_{I_{2}}\right\rangle_{2}\right\rangle_{J_{1}}-\left\langle\left\langle b, h_{I_{2}}\right\rangle_{2}\right\rangle_{K_{1}}=\sum_{l_{1}=1}^{j_{1}}\left\langle b, h_{J_{1}^{\left(l_{1}\right)}} \otimes h_{I_{2}}\right\rangle\left\langle h_{J_{1}^{\left(l_{1}\right)}}\right\rangle_{J_{1}} .
$$

It is now enough to fix $l_{1} \in\left\{1, \ldots, j_{1}\right\}$ and consider, for $g \in L_{x_{1}}^{p_{1}^{\prime}} L_{x_{2}}^{p_{2}^{\prime}}\left(X_{3}^{\prime}\right)$, the following dualized term

$$
\begin{aligned}
& \sum_{K_{1}} \sum_{L_{1}^{\left(j_{1}-l_{1}\right)}=K_{1}} \sum_{I_{2}}\left\{\left|\left\langle b, h_{L_{1}} \otimes h_{I_{2}}\right\rangle\right|,\right. \\
&\left.\sum_{\substack{I_{1}^{\left(i_{1}\right)}=K_{1} \\
J_{1}^{\left(l_{1}\right)}=L_{1}}}\left|L_{1}\right|^{-1 / 2}\left|a_{K_{1}, I_{1}, J_{1}}\right|\left|\left\langle f, h_{I_{1}} \otimes h_{I_{2}}\right\rangle\right| \sum_{K_{2} \supset I_{2}} \frac{\left|a_{K_{2}}\right|}{\left|K_{2}\right|}\left|\left\langle g, h_{J_{1}} \otimes h_{K_{2}}\right\rangle\right|\right\} .
\end{aligned}
$$

Notice that we can estimate

$$
\begin{aligned}
\sum_{K_{2} \supset I_{2}} \frac{\left|a_{K_{2}}\right|}{\left|K_{2}\right|}\left|\left\langle g, h_{J_{1}} \otimes h_{K_{2}}\right\rangle\right| & =\left\langle\sum_{K_{2} \supset I_{2}}\left|a_{K_{2}}\right|\left|\left\langle g, h_{J_{1}} \otimes h_{K_{2}}\right\rangle\right| \frac{1_{K_{2}}}{\left|K_{2}\right|}\right\rangle_{I_{2}} \\
& \leq\left|J_{1}\right|^{1 / 2}\left\langle\sum_{K_{2}}\left|a_{K_{2}}\right|\left\langle\left|\left\langle g, h_{K_{2}}\right\rangle_{2}\right|\right\rangle_{J_{1}} \frac{1_{K_{2}}}{\left|K_{2}\right|}\right\rangle_{I_{2}},
\end{aligned}
$$


and use the normalisation of $\left|a_{K_{1}, I_{1}, J_{1}}\right|$ to estimate

$$
\begin{aligned}
\left|L_{1}\right|^{-1 / 2}\left|a_{K_{1}, I_{1}, J_{1}}\right|\left|\left\langle f, h_{I_{1}} \otimes h_{I_{2}}\right\rangle\right| & \leq\left|L_{1}\right|^{-1 / 2} \frac{\left|I_{1}\right|^{1 / 2}\left|J_{1}\right|^{1 / 2}}{\left|K_{1}\right|}\left|\left\langle\left\langle\Delta_{K_{1}, i_{1}} f, h_{I_{2}}\right\rangle_{2}, h_{I_{1}}\right\rangle\right| \\
& \leq\left|L_{1}\right|^{-1 / 2} \frac{\left|J_{1}\right|^{1 / 2}}{\left|K_{1}\right|} \int_{I_{1}}\left|\left\langle\Delta_{K_{1}, i_{1}} f, h_{I_{2}}\right\rangle_{2}\right| .
\end{aligned}
$$

Now, by summing over $I_{1}, J_{1}$ we have reduced to bounding

$$
\begin{aligned}
\sum_{K_{1}} \sum_{L_{1}^{\left(j_{1}-l_{1}\right)}=K_{1}} \sum_{I_{2}}\left\{\left|\left\langle b, h_{L_{1}} \otimes h_{I_{2}}\right\rangle\right|,\right. & \\
& \left.\left|L_{1}\right|^{1 / 2}\left\langle\left|\left\langle\Delta_{K_{1}, i_{1}} f, h_{I_{2}}\right\rangle_{2}\right|\right\rangle_{K_{1}}\left\langle\sum_{K_{2}}\left|a_{K_{2}}\right|\left\langle\left|\left\langle g, h_{K_{2}}\right\rangle_{2}\right|\right\rangle_{L_{1}} \frac{1_{K_{2}}}{\left|K_{2}\right|}\right\rangle_{I_{2}}\right\} .
\end{aligned}
$$

We use Lemma 7.7 to dominate this by $\|b\|_{\mathrm{BMO}_{\mathcal{D} \text {,prod }}\left(X_{1}\right)} \leq\|b\|_{\mathrm{BMO}_{\text {prod }}\left(X_{1}\right)}$ multiplied with

$$
\left\|\left(\sum_{K_{1}} \sum_{I_{2}}\left[M_{\mathcal{D}^{1}}\left\langle\Delta_{K_{1}, i_{1}} f, h_{I_{2}}\right\rangle_{2}\right]^{2} \otimes \frac{1_{I_{2}}}{\left|I_{2}\right|}\right)^{1 / 2} M_{\mathcal{D}}\left(\sum_{K_{2}}\left|a_{K_{2}}\right|\left|\left\langle g, h_{K_{2}}\right\rangle_{2}\right| \otimes \frac{1_{K_{2}}}{\left|K_{2}\right|}\right)\right\|_{L^{1}\left(X_{1}^{\prime}\right)} .
$$

After using the estimate $\left|e_{2} e_{3}^{\prime}\right|_{X_{1}^{\prime}} \leq\left|e_{2}\right|_{X_{2}}\left|e_{3}^{\prime}\right|_{X_{3}^{\prime}}$ this term decouples naturally by Hölder's inequality. After this the proof can be ended using Lemma 7.5 and the $L_{x_{1}}^{p_{1}^{\prime}} L_{x_{2}}^{p_{2}^{\prime}}\left(X_{3}^{\prime}\right)$ boundedness of the paraproduct

$$
g \mapsto \sum_{K_{2}}\left|a_{K_{2}}\right|\left|\left\langle g, h_{K_{2}}\right\rangle_{2}\right| \otimes \frac{1_{K_{2}}}{\left|K_{2}\right|} .
$$

It only remains to estimate the terms in the big block, i.e., the terms

$$
\begin{aligned}
\sum_{\substack{\left.k_{1}, k_{2}\right) \neq(3,3) \\
3 \in\left\{k_{1}, k_{2}\right\}}} S_{1}\left(A_{k_{1}, k_{2}}\left(b, \pi_{2} f\right)\right) & +\sum_{k_{1}=1}^{2} \pi_{2}\left(A_{k_{1}}^{1}\left(b, S_{1} f\right)\right) \\
& -\sum_{\substack{\left(k_{1}, k_{2}\right) \neq(3,3) \\
3 \in\left\{k_{1}, k_{2}\right\}}} A_{k_{1}, k_{2}}\left(b, \pi_{2} S_{1} f\right)-\sum_{k_{1}=1}^{2} S_{1} \pi_{2}\left(A_{k_{1}}^{1}(b, f)\right) .
\end{aligned}
$$

Here we always have to pair two terms together, one with a plus and one with a minus sign, to induce cancellation to $b$. For example, we will consider

$$
S_{1}\left(A_{3,1}\left(b, \pi_{2} f\right)\right)-A_{3,1}\left(b, \pi_{2} S_{1} f\right) .
$$

Using that $\pi_{2} S_{1}=S_{1} \pi_{2}$ and the boundedness of $\pi_{2}$, it is enough to consider

$$
\begin{aligned}
& S_{1}\left(A_{3,1}(b, f)\right)-A_{3,1}\left(b, S_{1} f\right) \\
& =\sum_{\substack{K_{1} \\
I_{1}^{\left(i_{1}\right)}=J_{1}^{\left(j_{1}\right)}=K_{1}}} \sum_{K_{2}} a_{K_{1}, I_{1}, J_{1}}\left(\left\langle\left\langle b, h_{K_{2}}\right\rangle_{2}\right\rangle_{I_{1}}-\left\langle\left\langle b, h_{K_{2}}\right\rangle_{2}\right\rangle_{J_{1}}\right)\left\langle f, h_{I_{1}} \otimes h_{K_{2}}\right\rangle h_{J_{1}} \otimes h_{K_{2}} h_{K_{2}} .
\end{aligned}
$$

This can be bounded like the previous term, except it is simpler. This ends our proof. 
We note that the upper bound related to the lower bound of Proposition 5.3 - that is, the upper bound related to the diagonal $p_{1}=q_{1}$ and $p_{2}=q_{2}$ of Theorem 1.3 - is just the scalar-valued version of the above result. However, this scalar-valued result is very well-known. For general CZOs like here, the non-mixed norm version appears in [8]. The scalar-valued mixed-norm version could also directly be proved e.g. by extrapolating the estimate in [34], which is a two-weight version of [8].

Lastly, even if the bi-commutators $\left[T_{1},\left[b, T_{2}\right]\right]$ are the main topic of this paper, we record how the vector-valued estimate for the bi-commutator implies an estimate for a commutator with three CZOs. Establishing a dotted version of the space $L_{x_{3}}^{r_{3}}$ would require some modifications as in the bi-commutator case.

7.13. Proposition. Let $p_{1}, p_{2}, p_{3}, q_{3} \in(1, \infty), p_{3}>q_{3}$ and define $r_{3} \in(1, \infty)$ via

$$
\frac{1}{r_{3}}=\frac{1}{p_{3}}-\frac{1}{p_{3}}
$$

Let $T_{i}$ be a CZO in $\mathbb{R}^{d_{i}}, i=1,2,3$. Then we have

$$
\left\|\left[T_{1},\left[T_{2},\left[b, T_{3}\right]\right]\right] f\right\|_{L_{x_{1}}^{p_{1}} L_{x_{2}}^{p_{2}} L_{x_{3}}^{q_{3}}} \lesssim\|b\|_{\mathrm{BMO}_{\text {prod }, x_{1}, x_{2}}\left(L_{x_{3}}^{r_{3}}\right)}\|f\|_{L_{x_{1}}^{p_{1}} L_{x_{2}}^{p_{2}} L_{x_{3}}^{p_{3}}} .
$$

It is an interesting, but very complicated, problem to extend all of our results to tricommutators, or even higher order commutators.

\section{REFERENCES}

[1] A. Amenta, E. Lorist, M. Veraar, Rescaled extrapolation for vector-valued functions, Publ. Mat. 63 (2019) $155-182$

[2] A. Barron, J. M. Conde-Alonso, Y. Ou, G. Rey, Sparse domination and the strong maximal function. Adv. Math. 345 (2019), 1-26.

[3] J. Bourgain, Extension of a result of Benedek, Calderón and Panzone, Ark. Mat. 22 (1984) 91-95.

[4] S.-Y. A. Chang, R. Fefferman, A continuous version of duality of $H^{1}$ with BMO on the Bidisc, Ann. of Math. 112 (1980) 179-201.

[5] S.-Y. A. Chang, R. Fefferman, Some recent developments in Fourier analysis and $H^{p}$ theory on product domains, Bull. Amer. Math. Soc. 12 (1985) 1-43.

[6] R. Coifman, R. Rochberg, G. Weiss, Factorization theorems for Hardy spaces in several variables, Ann. of Math. (2) 103 (1976) 611-635.

[7] R. Coifman, P.-L. Lions, Y. Meyer, S. Semmes. Compensated compactness and Hardy spaces, J. Math. Pures Appl. 72 (3) (1993) 247-286.

[8] L. Dalenc, Y. Ou, Upper bound for multi-parameter iterated commutators Publ. Mat. 60 (2016) 191-220.

[9] G. David, J.-L. Journé, A boundedness criterion for generalized Calderón-Zygmund operators. Ann. of Math. (2) 120 (1984) 371-397.

[10] X.T. Duong, J. Li, Y. Ou, J. Pipher, B. Wick, Commutators of multi-parameter flag singular integrals and applications, Anal. PDE 12 (2019) 1325-1355.

[11] X.T. Duong, J. Li, Y. Ou, J. Pipher, B. Wick, Weighted Estimates of Singular Integrals and Commutators in the Zygmund Dilation Setting, preprint, arXiv:1905.00999, 2019

[12] X. T. Duong, J. Li, Y. Ou, B. Wick, D. Yang, Product BMO, Little BMO, and Riesz Commutators in the Bessel Setting, J. Geom. Anal. 28 (2018) 2558-2601.

[13] R. Fefferman, E. Stein, Singular integrals on product spaces, Adv. Math. 45 (1982) 117-143.

[14] S. H. Ferguson, M. T. Lacey, A characterization of product BMO by commutators, Acta Math. 189 (2002) 143-160.

[15] S. Ferguson, C. Sadosky, Characterizations of bounded mean oscillation on the polydisk in terms of Hankel operators and Carleson measures, J. Anal. Math. 81 (2000) 239-267.

[16] J. García-Cuerva, R. Macías, J. L. Torrea, The Hardy-Littlewood property of Banach lattices, Israel J. Math. 83 (1993) 177-201.

[17] I. Holmes, M. Lacey, B. Wick, Commutators in the two-weight setting, Math. Ann. 367 (2017) 51-80. 
[18] I. Holmes, M. Lacey, B. Wick, Bloom's inequality: commutators in a two-weight setting, Arch. Math. (Basel) 106 (2016) 53-63.

[19] I. Holmes, S. Petermichl, B. Wick, Weighted little bmo and two-weight inequalities for Journé commutators, Anal. PDE 11 (2018) 1693-1740.

[20] T. Hytönen, The sharp weighted bound for general Calderón-Zygmund operators, Ann. of Math. 175 (2012) 1473-1506.

[21] T. Hytönen, Representation of singular integrals by dyadic operators, and the $A_{2}$ theorem, Expo. Math. 35 (2017)166-205.

[22] T. Hytönen, The $L^{p}$-to- $L^{q}$ boundedness of commutators with applications to the Jacobian operator, preprint, arXiv:1804.11167, 2018.

[23] T. Hytönen, H. Martikainen, E. Vuorinen, Multi-parameter estimates via operator-valued shifts, Proc. Lond. Math. Soc. 119 (6) (2019) 1560-1597.

[24] T. Hytönen, J. van Neerven, M. Veraar, L. Weis, Analysis in Banach Spaces, Volume I: Martingales and Littlewood-Paley Theory, Springer-Verlag, 2016.

[25] T. Hytönen, J. van Neerven, M. Veraar, L. Weis, Analysis in Banach Spaces, Volume II: Probabilistic Methods and Operator Theory, Springer-Verlag, 2017.

[26] T. Hänninen, T. Hytönen, Operator-valued dyadic shifts and the T(1) theorem, Monatsh. Math. 180 (2016) 213-253.

[27] T. Iwaniec, Nonlinear commutators and Jacobians. Proceedings of the conference dedicated to Professor Miguel de Guzmán (El Escorial, 1996). J. Fourier Anal. Appl. 3 (1997), Special Issue, 775-796.

[28] S. Janson, Mean oscillation and commutators of singular integral operators, Ark. Mat. 16 (1978), no. 2, 263-270.

[29] M. Lacey, S. Petermichl, J. Pipher, B. Wick, Multiparameter Riesz commutators, Amer. J. Math. 131 (2009) 731-769.

[30] M. Lacey, S. Petermichl, J. Pipher, B. Wick, Iterated Riesz Commutators: A Simple Proof of Boundedness, Contemp. Math., 505, Amer. Math. Soc., Providence, RI, 2010, 171-178 .

[31] A. Lerner, S. Ombrosi, I. Rivera-Ríos, On pointwise and weighted estimates for commutators of Calderón-Zygmund operators, Adv. Math. 319 (2017) 153-181.

[32] A. Lerner, S. Ombrosi, I. Rivera-Ríos, Commutators of singular integrals revisited, Bull. London Math. Soc. 51 (2019) 107-119.

[33] K. Li, H. Martikainen, E. Vuorinen, Bloom type inequality for bi-parameter singular integrals: efficient proof and iterated commutators, Int. Math. Res. Not. IMRN (2019), rnz072, https:/ /doi.org/10.1093/imrn/rnz072.

[34] K. Li, H. Martikainen, E. Vuorinen, Bloom type upper bounds in the product BMO setting, J. Geom. Anal. (2019), https:/ /doi.org/10.1007/s12220-019-00194-3.

[35] K. Li, H. Martikainen, E. Vuorinen, Bilinear Calderón-Zygmund theory on product spaces, J. Math. Pures Appl., to appear, https:/ / doi.org/10.1016/j.matpur.2019.10.007.

[36] J. Lindenstrauss, L. Tzafriri, Classical Banach Spaces II: Function Spaces, Springer-Verlag, 1979.

[37] E. Lorist, Maximal functions, factorization, and the $\mathcal{R}$-boundedness of integral operators, Master's thesis, Delft University of Technology, Delft, the Netherlands, 2016.

[38] H. Martikainen, Representation of bi-parameter singular integrals by dyadic operators, Adv. Math. 229 (2012) 1734-1761.

[39] Z. Nehari, On bounded bilinear forms, Ann. of Math. 65 (1957) 153-162.

[40] J. L. Rubio de Francia, Martingale and integral transforms of Banach space valued functions, Lectures Notes in Mathematics 1221, Springer-Verlag, 1985, pp. 195-222.

[41] Y. Ou, Multi-parameter singular integral operators and representation theorem, Rev. Mat. Iberoam. 33 (2017) 325-350.

[42] Y. Ou, S. Petermichl, E. Strouse, Higher order Journé commutators and characterizations of multiparameter BMO, Adv. Math. 291 (2016) 24-58.

[43] S. Petermichl, Dyadic shifts and a logarithmic estimate for Hankel operators with matrix symbol, C. R. Acad. Sci. Paris Sér. I Math. 330 (2000) 455-460.

[44] C. Segovia, J. L. Torrea, Vector-valued commutators and applications, Indiana Univ. Math. J. 38 (1989) 959-971. 
(E.A., T.H., H.M, \& T.O.) DePARTMENT OF MATHEMATICS AND STATISTICS, UniVERSity Of HeLSinKi, P.O.B. 68, FI-00014 UNIVERSITY OF HELSINKI, FINLAND

E-mail address: emil.airta@helsinki.fi

E-mail address: tuomas.hytonen@helsinki.fi

E-mail address: henri.martikainen@helsinki.fi

E-mail address: tuomas.v.oikari@helsinki.fi

(K.L.) Center for Applied Mathematics, Tianjin University, Weijin Road 92, 300072 Tianjin, CHINA

E-mail address: kliet ju.edu.cn 